Nilpotent blocks of quasisimple groups for odd primes

An, Jianbei and Eaton, Charles W.

2009

MIMS EPrint: 2009.64

Manchester Institute for Mathematical Sciences

School of Mathematics

The University of Manchester

\footnotetext{
Reports available from: http://eprints.maths.manchester.ac.uk/

And by contacting: The MIMS Secretary

School of Mathematics

The University of Manchester

Manchester, M13 9PL, UK
} 


\title{
Nilpotent blocks of quasisimple groups for odd primes ${ }^{1}$
}

\author{
Jianbei An and Charles W. Eaton
}

\begin{abstract}
We investigate the nilpotent blocks of positive defect of the quasisimple groups for odd primes. In particular, it is shown that every nilpotent block of a quasisimple group has abelian defect groups. A conjecture of Puig concerning the recognition of nilpotent blocks is also shown for these groups.
\end{abstract}

\section{Introduction}

Let $G$ be a finite group and $k$ an algebraically closed field of odd characteristic $p$. A block $B$ of $k G$ with defect group $D$ is said to be nilpotent if for each $Q \leq D$ and each block $b_{Q}$ of $C_{G}(Q)$ with Brauer correspondent $B$ we have that $N_{G}\left(Q, b_{Q}\right) / C_{G}(Q)$ is a $p$-group, where $N_{G}\left(Q, b_{Q}\right)$ is the stabilizer of $b_{Q}$ under conjugation in $N_{G}(Q)$. In the case of the principal block $B_{0}, D$ is a Sylow $p$-subgroup of $G$ and $N_{G}\left(Q, b_{Q}\right)=N_{G}(Q)$ for each $Q \leq D$, so that $B_{0}$ is nilpotent if and only if $G$ is $p$-nilpotent (i.e., $G$ has a normal $p$-complement). Note that every block of defect zero must be nilpotent, and the classification of blocks of defect zero for finite simple groups has been the subject of a separate program of research, culminating in [21]. Hence we give attention here only to blocks with non-central defect groups.

Explicit characterizations of nilpotent blocks are obtained for classical groups, and these are used to prove:

Theorem 1.1 Let $G$ be a finite quasisimple group and let $B$ be a nilpotent p-block of $G$ with $p$ odd. Then $B$ has abelian defect groups.

The second main result concerns the conjecture of Puig which states that a block $B$ of $G$ is nilpotent if and only if $l\left(b_{Q}\right)=1$ for each $p$-subgroup $Q$ and each block $b_{Q}$ of $C_{G}(Q)$ with Brauer correspondent $B$ (where $l\left(b_{Q}\right)$ is the number of irreducible Brauer characters in $b_{Q}$ ). The necessary condition for nilpotency is well-known. The converse is known for blocks with abelian defect groups (see [30]), and is also known to be a consequence of Alperin's weight conjecture (see [33]). We prove that:

Theorem 1.2 Let $G$ be a finite quasisimple group and let $B$ be a p-block of $G$ with $p$ odd. Then $B$ is nilpotent if and only if $l\left(b_{Q}\right)=1$ for each $p$-subgroup $Q$ and each block $b_{Q}$ of $C_{G}(Q)$ with $\left(b_{Q}\right)^{G}=B$.

The main part of the paper concerns the representation theory of finite groups of Lie type in non-defining characteristic, and makes use of the examination of subpairs of blocks of classical groups given in [17]. The exceptional groups of Lie type are then treated by examination of the centralizer of an element of the centre of a defect group, and the results for the classical groups applied.

\footnotetext{
${ }^{1}$ The first author is supported by the Marsden Fund (of New Zealand), via award number UOA 0721 and the second author is supported by a Royal Society University Research Fellowship
} 
In Section 2 we review the basic notation regarding blocks, give some general results concerning nilpotent blocks, particularly with regard to block domination, and also prove some technical lemmas which will be useful later on. In Section 3 we consider the alternating groups and their covering groups. Here we have able to give a rather complete description of the nilpotent blocks. The covering groups of the sporadic simple groups are treated in Section 4. We give some basic notation used for the classical groups in Section 5. In Section 6 we give a treatment of the general linear and unitary groups, where again we are able to give a full characterization of the nilpotent blocks. In Section 7 we state the set of properties which are central to the study of the nilpotent blocks of the groups of Lie type. These are rather technical conditions, none of which can be satisfied by a nilpotent block with non-abelian defect groups, which amongst other things allow us to use inductive argument when studying the exceptional groups. That these conditions hold for the classical groups is the content of Section 8, and for the exceptional groups is the content of Section 9.

\section{Notation and general results}

Let $G$ be a finite group and $p$ a prime. Although the classification concerns only blocks with respect to a field of characteristic $p$, we use methods from ordinary character theory, for example canonical characters, and so must use a $p$-modular system. Let $\mathcal{O}$ be a local discrete valuation ring, complete with respect to the $p$-adic valuation, with field of fractions $K$ of characteristic zero and algebraically closed residue field $k=\mathcal{O} / J(\mathcal{O})$ of characteristic $p$. We assume that $\mathcal{O}$ contains a primitive $|G|$ th root of unity. Write $\operatorname{Blk}(G)$ for the set of blocks of $\mathcal{O} G$ and denote by $B_{0}(G)$ the principal block of $G$.

Let $N$ be a normal subgroup of $G$ and write $\operatorname{Irr}(G)$ the set of irreducible $K$ characters of $G$. For $\theta \in \operatorname{Irr}(N)$, we denote by $\operatorname{Irr}(G \mid \theta)$ the subset of $\operatorname{Irr}(G)$ consisting of characters covering $\theta$. We denote by $\operatorname{Irr}(B)$ the set of irreducible characters belonging to $B, k(B)=|\operatorname{Irr}(B)|$, and combine with the above notation freely.

Let $B$ be a $p$-block of a finite group $G$. A $B$-subgroup is a subpair $\left(Q, b_{Q}\right)$, where $Q$ is a $p$-subgroup of $G$ and $b_{Q}$ is a block of $Q C_{G}(Q)$ with Brauer correspondent $\left(b_{Q}\right)^{G}=B$. The $B$-subgroups with $|Q|$ maximized are called the Sylow $B$-subgroups, and they are the $B$-subgroups for which $Q$ is a defect group for $B$. Recall that the canonical character of $b_{Q}$ is the unique irreducible character in $b_{Q}$ with $Q$ in its kernel. This will be a valuable tool when comparing subpairs of a group with those of a normal subgroup.

A useful, and well-known, result is the following:

Proposition 2.1 Let $B$ be a block of a finite group $G$. Suppose a defect group $D$ of $B$ is abelian. Then $B$ is nilpotent if and only if $N_{G}\left(D, b_{D}\right)=C_{G}(D)$, where $\left(D, b_{D}\right)$ is a Sylow B-subgroup.

In general, we cannot say very much about the relationship between nilpotency of blocks and nilpotency of covered blocks, and this is a main reason behind the difficulty of the classification of nilpotent blocks of groups of Lie type.

However, we do have the following lemma by [24, Proposition 6.5]. 
Lemma 2.2 Let $N$ be a normal subgroup of a finite group $G$ such that $G / N$ is a pgroup. Suppose that $B$ is a block of $G$ and that $b \in \operatorname{Blk}(N)$ is covered by $B$. Then $B$ is nilpotent if and only if $b$ is nilpotent.

We note that the analogous result does not hold if $G / N$ is not a $p$-group. There are many examples of non-nilpotent blocks covering nilpotent blocks, but there are also examples of nilpotent blocks covering non-nilpotent blocks, such as the following (which came to light during a conversation with Radha Kessar):

Example 2.3 Let $G=\operatorname{PGL}(3,7), N=P S L(3,7)$ and $p=2$, so that $[G: N]=3$. Then $N$ has a unique block $b$ with defect group $D \cong \mathbb{Z}_{2} \times \mathbb{Z}_{2}$ and $b$ is not nilpotent. Moreover, $b$ is covered by a nilpotent block $B$ of $G$.

Note that $C_{N}(D)=\mathbb{Z}_{6} \times \mathbb{Z}_{2}$. Let $\operatorname{Irr}^{0}\left(C_{N}(D)\right)$ be the subset of $\operatorname{Irr}\left(C_{N}(D)\right)$ consisting of characters of $C_{N}(D)$ whose kernel contains $D$. Then $\left|\operatorname{Irr}^{0}\left(C_{N}(D)\right)\right|=3$. In addition, $C_{N}(D)$ has a unique character (the trivial character) $\xi \in \operatorname{Irr}^{0}\left(C_{N}(D)\right)$ such that $N_{N_{N}(D)}(\xi)=N_{N}(D)$, and two characters $\xi \in \operatorname{Irr}^{0}\left(C_{N}(D)\right)$ such that $N_{N_{N}(D)}(\xi)=$ $C_{N}(D) .3$. It follows that $N$ has exactly one block $b$ with a defect group $D$ and $b$ is non-nilpotent, as $N_{N}\left(D, b_{D}\right)=C_{N}(D) .3$ for a Sylow $b$-subgroup $\left(D, b_{D}\right)$.

Moreover, $C_{G}(D)=\mathbb{Z}_{6} \times \mathbb{Z}_{6}, N_{G}(D) / C_{G}(D) \cong S_{3}$ and $\left|\operatorname{Irr}^{0}\left(C_{G}(D)\right)\right|=9$. In addition, $C_{G}(D)$ has a unique character (the trivial character) $\xi \in \operatorname{Irr}^{0}\left(C_{G}(D)\right)$ such that $N_{N_{G}(D)}(\xi)=N_{G}(D)$, and eight characters $\xi \in \operatorname{Irr}^{0}\left(C_{G}(D)\right)$ such that $N_{N_{G}(D)}(\xi)=$ $C_{G}(D)$. It follows that $G$ has exactly one block $B$ with a defect group $D$ and $B$ is nilpotent, as $N_{G}\left(D, b_{D}\right)=C_{G}(D)$ for a Sylow $b$-subgroup $\left(D, b_{D}\right)$. Since $b$ is covered by a block of $G$ with a defect group $D$, it follows that $b$ is covered by $B$.

Recall that for $N \triangleleft G$, a block $B$ of $G$ is said to dominate the block $\bar{B}$ of $G / N$ if the inflation to $G$ of an irreducible character in $\bar{B}$ lies in $B$.

The following lemma follows by [33, Lemma 2].

Lemma 2.4 Let $Z$ be a central p-subgroup of a finite group $G, B \in \operatorname{Blk}(G)$ and $\bar{B}$ the block of $\bar{G}:=G / Z$ dominated by $B$. Then $B$ is nilpotent if and only if $\bar{B}$ is nilpotent.

Let $Z$ be a central $p^{\prime}$-subgroup of a finite group $G$, and write $\bar{H}=H Z / Z$, where $H \leq G$. Let $\bar{B} \in \operatorname{Blk}(\bar{G})$. There is a unique block $B \in \operatorname{Blk}(G)$ dominating $\bar{B}$. By $[25$, Theorem 5.8.8], $\operatorname{Irr}(B)=\operatorname{Irr}(\bar{B})$ and if $D$ is a defect group of $B$, then $D Z / Z \cong D$ is a defect group of $\bar{B}$.

If $Q$ is a $p$-subgroup of $G$, then $C_{\bar{G}}(\bar{Q})=C_{G}(Q) / Z$ (since $Z$ is a central $p^{\prime}$-subgroup). Let $\left(\bar{Q}, b_{\bar{Q}}\right)$ be a $\bar{B}$-subgroup. Then $\bar{Q}=Q Z / Z$ for a unique $p$-subgroup $Q$ of $G$. Since $C_{\bar{G}}(\bar{Q})=C_{G}(Q) / Z$, we may consider the unique subpair $\left(Q, b_{Q}\right)$ with $b_{Q}$ dominating $b_{\bar{Q}}$, which we call the Brauer pair dominating $\left(\bar{Q}, b_{\bar{Q}}\right)$.

We show that $\left(Q, b_{Q}\right)$ must be a $B$-subgroup, and that dominance of subpairs respects the usual partial order on $B$-subgroups:

Lemma 2.5 Let $Z$ be a central $p^{\prime}$-subgroup of a finite group $G$, and let $\left(\bar{Q}, b_{\bar{Q}}\right)$ and $\left(\bar{P}, b_{\bar{P}}\right)$ be $\bar{B}$-subgroups, where $\bar{B}$ is the block of $\bar{G}$ dominated by $B$. Suppose $\left(Q, b_{Q}\right)$ and $\left(P, b_{P}\right)$ are subpairs of $G$ dominating $\left(\bar{Q}, b_{\bar{Q}}\right)$ and $\left(\bar{P}, b_{\bar{P}}\right)$, respectively. Then $\left(\bar{Q}, b_{\bar{Q}}\right) \leq$ $\left(\bar{P}, b_{\bar{P}}\right)$ if and only if $\left(Q, b_{Q}\right) \leq\left(P, b_{P}\right)$. In particular, $\left(Q, b_{Q}\right)$ is a B-subgroup. 
Proof: Let $F$ be a splitting field of characteristic $p$ of $G$, and $\mu_{Z}$ the map from $F G$ to $F \bar{G}$ defined by $\mu_{Z}\left(\sum_{x \in G} \alpha_{x} x\right)=\sum_{x \in G} \alpha_{x} \bar{x}$, where $\bar{x}=\mu_{Z}(x)$. For $H \leq G$, let $\mathcal{C} \ell(G \mid H)$ be the $H$-orbits of $G$ under conjugation. Then $\{[C]: C \in \mathcal{C} \ell(G \mid H)\}$ forms a $F$-basis of the fixed point set $(F G)^{H}$, where $[C]:=\sum_{x \in C} x$.

If $H$ is a $p$-subgroup, then $(F G)^{H}=F C_{G}(H) \oplus I^{H}(F G)$ as vector spaces, where $I^{H}(F G)=\sum_{W<H}(F G)_{W}^{H}$ is an ideal of $(F G)^{H}$ and $\{[C]: C \in \mathcal{C} \ell(G \mid H),|C| \neq 1\}$ forms an $F$-basis of $I^{H}(F G)$. Thus $B r_{H}([C]):=\left[C \cap C_{G}(H)\right]$ gives the natural algebra homomorphism from $(F G)^{H}$ onto $F C_{G}(H)$ with kernel $I^{H}(F G)$. Similarly,

$$
(F \bar{G})^{H}=F\left(C_{G}(H) / Z\right) \oplus I^{H}(F \bar{G})
$$

and $\mu_{Z}: I^{H}(F G) \rightarrow I^{H}(F \bar{G})$ is an isomorphism of algebras. Now

$$
B r_{\bar{H}}:(F \bar{G})^{H} \rightarrow F\left(C_{G}(H) / Z\right)
$$

and $\mu_{Z}: F C_{G}(H) \rightarrow F\left(C_{G}(H) / Z\right)$, so $\mu_{Z} \circ B r_{H}=B r_{\bar{H}} \circ \mu_{Z}$.

Suppose $\left(\bar{Q}, b_{\bar{Q}}\right) \triangleleft\left(\bar{P}, b_{\bar{P}}\right)$, so that $\bar{Q} \triangleleft \bar{P}$. Since $\bar{Q}=Q Z / Z$ and $\bar{P}=P Z / Z$ for p-subgroups $Q, P$ of $G$ and since $Q$ is the only Sylow $p$-subgroup of $Q Z$, it follows that $Q \triangleleft P$. Since $P Z=P \times Z$ and $b_{\bar{Q}}$ is $\bar{P}$-invariant, it follows that for any $y \in P$ $b_{Q}^{y}$ is a block of $C_{G}(Q)$ dominating $b_{\bar{Q}}$, so that by the uniqueness, $b_{Q}^{y}=b_{Q}$ and $b_{Q}$ is $P$-invariant. Now

$$
\mu_{Z}\left(B r_{P}\left(b_{Q}\right) b_{P}\right)=\mu_{Z}\left(B r_{P}\left(b_{Q}\right)\right) b_{\bar{P}}=B r_{\bar{P}}\left(\mu_{Z}\left(b_{Q}\right)\right) b_{\bar{P}}=B r_{\bar{P}}\left(b_{\bar{Q}}\right) b_{\bar{P}}=b_{\bar{P}} \neq 0,
$$

so that $B r_{P}\left(b_{Q}\right) b_{P} \neq 0$ and $B r_{P}\left(b_{Q}\right) b_{P}=b_{P}$. It follows that $\left(Q, b_{Q}\right) \triangleleft\left(P, b_{P}\right)$. Using induction we have that $\left(Q, b_{Q}\right) \leq\left(P, b_{P}\right)$ if $\left(\bar{Q}, b_{\bar{Q}}\right) \leq\left(\bar{P}, b_{\bar{P}}\right)$.

Suppose $\left(Q, b_{Q}\right) \triangleleft\left(P, b_{P}\right)$, so that $Q \triangleleft P$ and $\bar{Q} \triangleleft \bar{P}$. Since $b_{Q}$ is $P$-invariant, it follows that $b_{\bar{Q}}$ is $\bar{P}$-invariant. Since $B r_{P}\left(b_{Q}\right) b_{P}=b_{P}$, it follows that

$$
B r_{\bar{P}}\left(b_{\bar{Q}}\right) b_{\bar{P}}=\mu_{Z}\left(B r_{P}\left(b_{Q}\right) b_{P}\right)=\mu_{Z}\left(b_{P}\right)=b_{\bar{P}},
$$

so that $\left(\bar{Q}, b_{\bar{Q}}\right) \triangleleft\left(\bar{P}, b_{\bar{P}}\right)$. Similarly, if $\left(Q, b_{Q}\right) \leq\left(P, b_{P}\right)$, then $\left(\bar{Q}, b_{\bar{Q}}\right) \leq\left(\bar{P}, b_{\bar{P}}\right)$.

We obtain as a consequence:

Proposition 2.6 Let $G$ be a finite group, $Z \leq Z(G)$ and $\bar{G}=G / Z$. Suppose $\bar{B} \in$ $\operatorname{Blk}(\bar{G})$ and $B \in \operatorname{Blk}(G)$ dominating $\bar{B}$. Then $\bar{B}$ is nilpotent if and only if $B$ is nilpotent.

Proof: Write $Z_{p}=O_{p}(Z), Z_{p^{\prime}}=O_{p^{\prime}}(Z), G_{1}=G / Z_{p^{\prime}}$ and let $B_{1} \in \operatorname{Blk}\left(G_{1}\right)$ be the unique block of $G_{1}$ dominated by $B$. Then $\operatorname{Irr}\left(B_{1}\right)=\operatorname{Irr}(B)$, and $B_{1}$ dominates $\bar{B}$.

By Lemma 2.4, $\bar{B}$ is nilpotent if and only if $B_{1}$ is nilpotent. Hence we suppose $\bar{B}=B_{1}$ and $Z=Z_{p^{\prime}}$.

Let $\left(\bar{D}, b_{\bar{D}}\right)$ be a Sylow $\bar{B}$-subgroup and $\left(D, b_{D}\right)$ the unique $B$-subgroup dominating $\left(\bar{D}, b_{\bar{D}}\right)$. Note that $\left(D, b_{D}\right)$ is a Sylow $B$-subgroup.

Suppose $\left(\bar{Q}, b_{\bar{Q}}\right)$ is a $B$-subgroup and $\left(Q, b_{Q}\right)$ is the $B$-subgroup dominating $\left(\bar{Q}, b_{\bar{Q}}\right)$. If $\bar{x} \in N_{\bar{G}}\left(\bar{Q}, b_{\bar{Q}}\right)$, then $\bar{x}=x Z$ for some $x \in G$, and $x Z \subseteq N_{G}(Q)$. Since $\mu_{Z}\left(b_{Q}\right)=b_{\bar{Q}}$ and $\mu_{Z}\left(b_{Q}^{x}\right)=b_{\bar{Q}}^{\bar{x}}=b_{\bar{Q}}$, it follows that $b_{Q}$ and $b_{Q}^{x}$ both are blocks of $C_{G}(Q)$ dominating 
$b_{\bar{Q}}$ and $b_{Q}=b_{Q}^{x}$ by uniqueness. Thus $x \in N_{G}\left(Q, b_{Q}\right)$ and $N_{\bar{G}}\left(\bar{Q}, b_{\bar{Q}}\right)=N_{G}\left(Q, b_{Q}\right) / Z$. Since $Z \leq C_{G}(Q)$ and $C_{\bar{G}}(\bar{Q})=C_{G}(Q) / Z$, it follows that

$$
N_{\bar{G}}\left(\bar{Q}, b_{\bar{Q}}\right) / C_{\bar{G}}(\bar{Q}) \bar{Q} \cong N_{G}\left(Q, b_{Q}\right) / C_{G}(Q) Q .
$$

Suppose $\bar{B}$ is not nilpotent, so that there is some $\bar{B}$-subgroup $\left(\bar{Q}, b_{\bar{Q}}\right)$ such that $N_{\bar{G}}\left(\bar{Q}, b_{\bar{Q}}\right) / C_{\bar{G}}(\bar{Q}) \bar{Q}$ is not a $p$-group. Thus $N_{G}\left(Q, b_{Q}\right) / C_{G}(Q) Q$ is not a $p$-group and $B$ is not nilpotent.

Suppose $B$ is not nilpotent, so that $N_{G}\left(Q, b_{Q}\right) / C_{G}(Q) Q$ is not a $p$-group for some $B$-subgroup $\left(Q, b_{Q}\right)$. We may suppose $\left(Q, b_{Q}\right) \leq\left(D, b_{D}\right)$. Thus $\bar{Q}=Q Z / Z \leq \bar{D}$, and $\left(\bar{Q}, b_{\bar{Q}}\right) \leq\left(\bar{D}, b_{\bar{D}}\right)$ for a unique $\bar{B}$-subgroup $\left(\bar{Q}, b_{\bar{Q}}\right)$. Let $\left(Q, b_{Q}^{\prime}\right)$ be a $B$-subgroup dominating $\left(\bar{Q}, b_{\bar{Q}}\right)$. By Lemma $2.5\left(Q, b_{Q}^{\prime}\right) \leq\left(D, b_{D}\right)$, so that by the uniqueness $\left(Q, b_{Q}^{\prime}\right)=$ $\left(Q, b_{Q}\right)$. Thus $N_{\bar{G}}\left(\bar{Q}, b_{\bar{Q}}\right) / C_{\bar{G}}(\bar{Q}) \bar{Q} \cong N_{G}\left(Q, b_{Q}\right) / C_{G}(Q) Q$, and $N_{\bar{G}}\left(\bar{Q}, b_{\bar{Q}}\right) / C_{\bar{G}}(\bar{Q}) \bar{Q}$ is not a $p$-group. It follows that $\bar{B}$ is not nilpotent.

When considering groups of Lie type, we will often examine the centralisers of $p$ elements, which may be written as central products of groups. By a central product $G_{1} \circ_{Z} G_{2}$ of $G_{1}$ and $G_{2}$ over $Z \leq Z\left(G_{1}\right) \cap Z\left(G_{2}\right)$, we mean that $G_{1} \circ_{Z} G_{2}=G_{1} G_{2}$, where $G_{1}$ and $G_{2}$ are subgroups of $G_{1} \circ_{Z} G_{2}$ with $G_{1} \cap G_{2}=Z$ and $\left[G_{1}, G_{2}\right]=1$. When it is clear what $Z$ is, we write $G_{1} \circ G_{2}=G_{1} \circ Z G_{2}$. Note that $G_{1} \circ G_{2} \cong\left(G_{1} \times G_{2}\right) /\left\{\left(z, z^{-1}\right)\right.$ : $z \in Z\}$. For $\chi_{i} \in \operatorname{Irr}\left(G_{i}\right)$ such that $\chi_{1}$ and $\chi_{2}$ both cover the same irreducible character of $Z$, we may define $\chi_{1} \circ \chi_{2} \in \operatorname{Irr}\left(G_{1} \circ G_{2}\right)$ so that $\chi_{1} \chi_{2} \in \operatorname{Irr}\left(G_{1} \times G_{2}\right)$ is the inflation of $\chi_{1} \circ \chi_{2}$. We refer to $\chi_{1} \circ \chi_{2}$ as the central product of $\chi_{1}$ and $\chi_{2}$.

We will need the following technical lemma in certain cases in relation to Property 7.1 (a) in Section 7 holds.

Lemma 2.7 For $i=1,2$, let $G_{i}$ be a finite group, $G_{1} \circ G_{2}$ a central product of $G_{1}$ and $G_{2}$ over $Z \leq Z\left(G_{1}\right) \cap Z\left(G_{2}\right)$ and $N_{i}$ a normal subgroup of $G_{i}$ such that $G_{i} / N_{i}$ is abelian, and let $N:=N_{1} \times N_{2} \leq G \leq G_{1} \circ G_{2}$ such that $\pi_{i}(G)=G_{i} / Z$, where $\pi_{i}:\left(G_{1} \circ G_{2}\right) \rightarrow G_{i} / Z$ is the canonical projection. Let $\theta_{i} \in \operatorname{Irr}\left(N_{i}\right)$ such that $\theta_{2}$ has an extension $\tilde{\theta}_{2}$ to $G_{2}$, and let $\theta=\theta_{1} \times \theta_{2}$ and $\varphi \in \operatorname{Irr}(G \mid \theta)$.

(i) There exist $\psi_{1} \in \operatorname{Irr}\left(G_{1}\right)$ and $\lambda \in \operatorname{Irr}\left(G_{2} / N_{2}\right)$ covering the same irreducible character of $Z$, such that the restriction $\left.\left(\psi_{1} \circ\left(\tilde{\theta}_{2} \lambda\right)\right)\right|_{G}$ of $\psi_{1} \circ\left(\tilde{\theta}_{2} \lambda\right)$ is equal to $\varphi$. Moreover, if $\psi \in \operatorname{Irr}\left(G_{1} \circ G_{2} \mid \varphi\right)$, then $\left.\psi\right|_{G}=\varphi$.

(ii) If further $Z \cap N_{2}=1$, then $\lambda$ in (i) may be chosen with $Z N_{2} / N_{2}$ in its kernel, so that it may be regarded as a character of $G_{2} / N_{2} Z$.

(iii) Suppose that $Z \cap N_{2}=1$. If we have $y \in \operatorname{Aut}\left(G_{1} \circ G_{2}\right)$ such that $y$ centralizes $G_{1}$, stabilizes $G, G_{2}$ and $\tilde{\theta}_{2}$, and $g_{2}^{y} \in g_{2} N_{2} Z$ for any $g_{2} \in G_{2}$, then $y$ stabilizes $\varphi$.

Proof: (i) We first claim that we may suppose $Z \leq G$. For since $Z \leq Z\left(G_{1} \circ G_{2}\right)$, we have that $G Z$ is a central product over $G \cap Z$. Now $\left.\varphi\right|_{G \cap Z}=\varphi(1) \alpha$ for some $\alpha \in \operatorname{Irr}(G \cap Z)$. Since $Z$ is abelian, there exists $\tilde{\alpha} \in \operatorname{Irr}(Z)$ extending $\alpha$. Then $\tilde{\varphi}=\varphi \tilde{\alpha}$ is an extension of $\varphi$ covering $\theta$. If $\psi_{1} \in \operatorname{Irr}\left(G_{1}\right)$ and $\lambda \in \operatorname{Irr}\left(G_{2} / N_{2}\right)$ such that $\psi_{1}$ and $\lambda$ cover the same irreducible character of $Z$ and $\left.\left(\psi_{1} \circ \tilde{\theta}_{2} \lambda\right)\right|_{G Z}=\tilde{\varphi}$, then $\left.\left(\psi_{1} \circ \tilde{\theta}_{2} \lambda\right)\right|_{G}=\varphi$, and similarly for the final statement, proving the claim.

Similarly, $\left(N_{1} \times N_{2}\right) Z=\left(N_{1} Z\right) \circ\left(N_{2} Z\right)$ and $\varphi$ covers an irreducible character $\xi \in \operatorname{Irr}\left(\left(N_{1} Z\right) \circ\left(N_{2} Z\right) \mid \theta\right)$ with $\xi=\xi_{1} \circ \xi_{2}$ for some $\xi_{i} \in \operatorname{Irr}\left(N_{i} Z \mid \theta_{i}\right)$ covering 
the same irreducible character of $Z$. Note that $\left.\tilde{\theta}_{2}\right|_{N_{2} Z}$ is also an extension of $\theta_{2}$ to $N_{2} Z$. By Gallagher's theorem, $\left(\left.\tilde{\theta}_{2}\right|_{N_{2} Z}\right) \beta_{2}=\xi_{2}$ for some $\beta_{2} \in \operatorname{Irr}\left(N_{2} Z / N_{2}\right)$. Since $G_{2} / N_{2}$ is abelian, it follows that $\beta_{2}$ can be viewed as the restriction of a character $\beta \in \operatorname{Irr}\left(G_{2} / N_{2}\right)$, so $\xi_{2}=\left.\left(\tilde{\theta}_{2} \beta\right)\right|_{N_{2} Z}$. Write $\tilde{\xi}_{2}=\tilde{\theta}_{2} \beta$, so $\tilde{\xi}_{2}$ is an extension of $\xi_{2}$ to $G_{2}$

Let $M_{1}$ be a subgroup of $G_{1}$ such that $\xi_{1}$ has an extension $\tilde{\xi}_{1}$ to $M_{1}$ and $M_{1}$ is maximal with this property, that is, either $M_{1}=G_{1}$ or $\xi_{1}$ has no extension to $H_{1}$ for any $M_{1}<H_{1} \leq G_{1}$. Since $G_{1} /\left(N_{1} Z\right)$ is abelian, it follows that the inertia subgroup $I_{G_{1}}\left(\tilde{\xi}_{1}\right)$ equals $M_{1}$.

Let $M=\left(M_{1} \circ G_{2}\right) \cap G \leq G, M_{2}:=G_{2}$ and $\gamma \in \operatorname{Irr}\left(M_{1} \circ M_{2} \mid \xi\right)$. Then $G / M \cong G_{1} / M_{1}$ and $\gamma=\gamma_{1} \circ \gamma_{2}$ for some $\gamma_{i} \in \operatorname{Irr}\left(M_{i} \mid \xi_{i}\right)$. Since $\xi_{i}$ has an extension $\tilde{\xi}_{i}$ to $M_{i}$, it follows that $\gamma_{i}=\tilde{\xi}_{i} \lambda_{i}$ for some $\lambda_{i} \in \operatorname{Irr}\left(M_{i} / N_{i} Z\right)$, so that $\left.\gamma\right|_{M}$ is an extension of $\xi$ to $M$. Note that $M \leq I_{G}(\xi)$ and $\varphi$ also covers an extension of $\xi$ to $M$. Replacing $\gamma_{i}$ by $\gamma_{i} \alpha_{i}$ for some $\alpha_{i} \in \operatorname{Irr}\left(M_{i} / N_{i} Z\right)$ if necessary, we may suppose $\varphi \in \operatorname{Irr}\left(G|\gamma|_{M}\right)$.

Since $\left.\gamma_{1}\right|_{N_{1} Z}=\xi_{1}$ and $G_{2}$ stabilizes $\tilde{\xi}_{2}$ (and $G_{1} / N_{1} Z$ is abelian), it follows that $I_{G_{1}}\left(\gamma_{1}\right)=M_{1}, I_{G}(\gamma)=M$ and $I_{G_{1} \circ G_{2}}(\gamma)=M_{1} \circ G_{2}$. Let $\psi_{1}=\operatorname{Ind}_{M_{1}}^{G_{1}}\left(\gamma_{1}\right)$, so that

$$
\zeta:=\psi_{1} \circ \gamma_{2}=\operatorname{Ind}_{M_{1} \circ G_{2}}^{G_{1} \circ G_{2}}\left(\gamma_{1} \circ \gamma_{2}\right)
$$

$\operatorname{But} \varphi=\operatorname{Ind}_{M}^{G}\left(\left.\gamma\right|_{M}\right)$, so

$$
\left(\left.\zeta\right|_{G}, \varphi\right)_{G}=\left(\left.\zeta\right|_{M},\left.\gamma\right|_{M}\right)_{M}
$$

Since $M$ and $M_{1} \circ G_{2}$ are both normal in $G_{1} \circ G_{2}$ and $M \leq M_{1} \circ G_{2}$, it follows that $\left(M_{1} \circ G_{2}\right) \backslash\left(G_{1} \circ G_{2}\right) / M=\left(G_{1} \circ G_{2}\right) /\left(M_{1} \circ G_{2}\right) \cong G_{1} / M_{1} \cong G / M$. Note also $\left(M_{1} \circ G_{2}\right)^{t} \cap M=M$ for any $t \in G / M$. Hence the Mackey decomposition gives us

$$
\left.\zeta\right|_{M}=\sum_{t \in G / M}\left(\left.\left(\gamma_{1} \circ \gamma_{2}\right)^{t}\right|_{M}\right)=\sum_{t \in G / M}\left(\left(\left.\gamma\right|_{M}\right)^{t}\right)
$$

and so $\left(\left.\zeta\right|_{M},\left.\gamma\right|_{M}\right)_{M}=1$. Since $\zeta(1)=\varphi(1)=[G: M] \gamma(1)$, it follows that $\left.\zeta\right|_{G}=\varphi$.

Note that $I_{G_{1} \circ G_{2}}(\varphi)=G_{1} \circ G_{2}$ and $\varphi$ has an extension $\zeta$ to $G_{1} \circ G_{2}$. If $\psi \in$ $\operatorname{Irr}\left(G_{1} \circ G_{2} \mid \varphi\right)$, then by Gallagher's theorem, $\psi=\zeta \eta$ for some $\eta \in \operatorname{Irr}\left(\left(G_{1} \circ G_{2}\right) / G\right)$ and so $\left.\psi\right|_{G}=\varphi$.

(ii) First note that $Z N_{2}=Z \times N_{2}$ and $\left(N_{1} \times N_{2}\right) Z=\left(N_{1} \circ Z\right) \times N_{2}$. If $\xi \in$ $\operatorname{Irr}\left(\left(N_{1} \times N_{2}\right) Z \mid \theta\right)$, then $\xi=\left(\theta_{1} \circ \eta\right) \times \theta_{2}$ for some $\eta \in \operatorname{Irr}(Z)$. Thus we may suppose $\xi_{1}=\left(\theta_{1} \circ \eta\right) \in \operatorname{Irr}\left(N_{1} Z\right)$ and $\xi_{2}=\left(\theta_{2} \times 1_{Z}\right) \in \operatorname{Irr}\left(N_{2} Z\right)$, and take $\tilde{\xi}_{2}=\tilde{\theta}_{2}$ as an extension of $\xi_{2}$ to $G_{2}$. As shown in the proof of part (i) $\varphi=\left.\left(\psi_{1} \circ\left(\tilde{\theta}_{2} \lambda\right)\right)\right|_{G}$ for some $\psi_{1} \in \operatorname{Irr}\left(G_{1}\right)$ and $\lambda \in \operatorname{Irr}\left(G_{2} / N_{2} Z\right)$.

(iii) Since $y$ centralizes the factor group $G_{2} / Z N_{2}$, it follows that $y$ stabilizes $\lambda$, so does $\tilde{\theta}_{2} \lambda$. But $y$ centralizes $G_{1}$, so $y$ stabilizes $\psi_{1} \circ\left(\tilde{\theta}_{2} \lambda\right)$ and hence $y$ stabilizes $\varphi$.

\section{$3 \quad$ The alternating groups}

To handle the case $p=3$ we will need the following. The first lemma will be used in determining non-faithful nilpotent blocks of the double covers of alternating groups. Recall that a partition is self-associate if its Young diagram is symmetric. 
Lemma 3.1 Let $n$ be a positive integer. There is a self-associate 3 -core partition $\lambda \vdash n$ if and only if there is a positive integer $m$ such that $n=3 m^{2}+2 m$ or $n=3 m^{2}-2 m$.

Proof: We claim that the self-associate 3-cores, i.e., those Young diagrams which are symmetric about the leading diagonal and have no 3-hooks, are those which arise from partitions of the form

$$
\left(3 m, 3 m-2,3 m-4, \ldots, 3 m-2(m-1), m^{2},(m-1)^{2}, \ldots, 2^{2}, 1^{2}\right)
$$

and

$$
\left(3 m-2,3 m-4,3 m-6, \ldots, 3 m-2 m,(m-1)^{2},(m-2)^{2}, \ldots, 2^{2}, 1^{2}\right)
$$

for integers $m \geq 1$.

This may be seen directly from methodical construction of the possible Young diagrams. However, we give a formal proof here using [19]. For a fixed $t$, Garson, Kim and Stanton give a bijection $\phi$ between the set of $t$-cores and $\left\{n_{0}, n_{1}, \ldots, n_{t-1} \in \mathbb{Z}^{t}\right.$ : $\left.n_{0}+\cdots+n_{t-1}=0\right\}$, defined as follows. We of course only need to consider the case $t=3$. Let $\lambda$ be a 3 -core. We take the 3 -residue diagram, i.e., in the $(i, j)$ th cell of the Young diagram we put the residue of $j-i$ modulo 3 (see [23, p.84]). We also include the 0th column (with infinitely many entries), calling this the extended 3-residue diagram. Divide this into regions labelled by the integers as follows: the $(i, j)$ th cell lies in the region $r$ if $3(r-1) \leq j-i<3 r$. Say that a cell is exposed if it lies at the end of a row. Define $n_{i}$ to be the maximal $r$ such that an exposed cell with value $i$ lies in the region $r$ (the inclusion of the $0 t h$ row ensures the existence of such an $r$ ).

It is verified in [19] that $\phi$ does indeed give a bijection. It is also shown that $\lambda$ is selfassociate if and only if $\phi(\lambda)=\left(n_{0}, n_{1}, n_{2}\right)=\left(-n_{2},-n_{1},-n_{0}\right)$, i.e., if $\phi(\lambda)=(m, 0,-m)$ for some $m \in \mathbb{Z}$.

Suppose first that $m>0$. Then the end cell on the first row is labelled 0 , so the first row has length $\lambda_{1}=3(m-1)+1=3 m-2$. Since regions 0 and $-m$ lie below the leading diagonal, the end cells lying above the diagonal are all labelled 0 . Since $\lambda$ is a 3 -core, the difference between adjacent row lengths is at most 2 , hence the row lengths decrease in steps of two until the mth row (which has end cell on the leading diagonal). Since $\lambda$, is self adjoint, this determines the whole Young diagram and we are done in this case.

Suppose that $m \leq 0$. Then the end cell of the first row is labelled 2 , so the first row has length $\lambda_{1}=3 \mathrm{~m}$, and by a similar argument to the above the difference between adjacent row lengths is 2 until the $(m+1)$ th row (which has end cell below the leading diagonal). Again this determines $\lambda$, and we are done.

We now consider the analogue of the above lemma which will be used for faithful blocks. We write $\lambda \succ n$ for a bar partition of $n$ (i.e., a partition with distinct parts). Recall that a bar partition $\lambda \succ n$ is odd or even according as $n-r$ is odd or even, where $r$ is the number of parts in the partition. We refer to [26] for definitions of bars and $\bar{p}$-cores.

Lemma 3.2 Let $n$ be a positive integer. There is an even $\overline{3}$-core bar partition $\lambda \succ n$ if and only if there is a positive integer $m_{1}$ with $m_{1} \equiv 0,1 \bmod 4$ and $n=\left(3 m_{1}^{2}-m_{1}\right) / 2$ or a positive integer $m_{2}$ with $m_{2} \equiv 0,3 \bmod 4$ and $n=\left(3 m_{2}^{2}+m_{2}\right) / 2$. 
Proof: Determining the $\overline{3}$-core partitions is a little more straight-forward than determining 3 -core partitions, and the reader can easily verify that the $\overline{3}$-core bar partitions are precisely those of the form

$$
\lambda_{m}^{-}:=(3 m-2,3 m-5, \ldots, 3 m-2-3 i, \ldots, 4,1)
$$

or

$$
\lambda_{m}^{+}:=(3 m-1,3 m-4, \ldots, 3 m-1-3 i, \ldots, 5,2) .
$$

Note that $\lambda_{m}^{-} \succ\left(3 r^{2}-m\right) / 2$ and $\lambda_{m}^{+} \succ\left(3 m^{2}+m\right) / 2$. Also note that $\lambda_{m}^{-}$is even if and only if $m \equiv 0,1 \bmod 4 ; \lambda_{m}^{+}$is even if and only if $m \equiv 0,3 \bmod 4$.

Theorem 3.3 Let $n$ be an integer with $n \geq 5$ and $G=\hat{A}_{n}$, the double cover of $A_{n}$. Let $p$ be an odd prime. If $p \neq 3$, then $G$ does not possess a nilpotent $p$-block of positive defect. If $p=3$, then $G$ possesses a non-faithful nilpotent block of positive defect if and only if $n=3 m^{2}+2 m+3$ or $n=3 m^{2}-2 m+3$ for some positive integer $m$. Also if $p=3$, then $G$ possesses a faithful nilpotent block of positive defect if and only if $n=\left(3 m_{1}^{2}-m_{1}+6\right) / 2$ for a positive integer $m_{1}$ with $m_{1} \equiv 0,1 \bmod 4$ or $n=\left(3 m_{2}^{2}+m_{2}+6\right) / 2$ for a positive integer $m_{2}$ with $m_{2} \equiv 0,3 \bmod 4$. In each case the nilpotent blocks have defect groups of order 3 generated by (the preimage of) a 3-cycle.

Proof: The properties of $\hat{A}_{n}$ used here are described in $[20,5.2]$. We consider $\hat{A}_{n} \leq \hat{S}_{n}$, the double cover of the symmetric group. Write $Z=Z\left(\hat{S}_{n}\right)$ and $\bar{X}=X Z / Z$ whenever $X \leq \hat{S}_{n}$. For convenience of notation we write $\overline{\hat{S}_{n}}=S_{n}$ and $\overline{\hat{A}_{n}}=A_{n}$. Since we are taking $p$ odd, for every $p$-subgroup $Q \leq \hat{S}_{n}$ we have $C_{S_{n}}(\bar{Q})=\overline{C_{\hat{S}_{n}}(Q)}$ and $N_{S_{n}}(\bar{Q})=\overline{N_{\hat{S}_{n}}(Q)}$. Suppose that $B$ is a nilpotent $p$-block of $\hat{A}_{n}$ with non-trivial defect group $D$. Choose $y \in D$ of order $p$. Then $y Z$ is a product of say $t$ disjoint $p$-cycles, fixing the other $n-p t$ points. Then $C_{S_{n}}(y Z) \cong\left(\mathbb{Z}_{p}\left\langle S_{t}\right) \times S_{n-p t}\right.$, and so $C_{\hat{A}_{n}}(y)$ contains a normal elementary abelian $p$-group $R$ such that $\bar{R}$ is generated by $t$ disjoint $p$-cycles. Now $R$ is contained in a conjugate of $D$, and so in particular $D$ contains an element $x$ for which $x Z$ is a $p$-cycle. Write $Q=\langle x\rangle$. We have $C_{A_{n}}(\bar{Q}) \cong \bar{Q} \times A_{n-p}$. By $[20,5.2 .6]$ we have $C_{\hat{A}_{n}}(Q) \cong Q \times \hat{A}_{n-p}$ (the point here being that the central extension of $A_{n-p}$ does not split). We have $N_{S_{n}}(\bar{Q}) \cong N_{S_{p}}(\bar{Q}) \times S_{n-p}$ and $N_{A_{n}}(\bar{Q}) \cong\left(N_{A_{p}}(\bar{Q}) \times A_{n-p}\right)\langle\bar{a}\rangle$ where $\bar{a}^{2}=1$. Note that $\left[N_{\hat{A}_{n}}(Q): C_{\hat{A}_{n}}(Q)\right]=p-1$.

The $p$-blocks of $C_{\hat{A}_{n}}(Q)$ are in 1-1 correspondence with the $p$-blocks of $\hat{A}_{n-p}$ and the action of $N_{\hat{A}_{n}}(Q)$ on these blocks is determined by the action of $\hat{S}_{n-p}$ on the blocks of $\hat{A}_{n-p}$. Hence for each block $b_{Q}$ of $C_{\hat{A}_{n}}(Q)$ we have $\left[N_{\hat{A}_{n}}\left(Q, b_{Q}\right): C_{\hat{A}_{n}}(Q)\right]=(p-1) / 2$ or $p-1$.

If $p>3$, then this shows that $N_{\hat{A}_{n}}\left(Q, b_{Q}\right) / C_{\hat{A}_{n}}(Q)$ is not a $p$-group, contradicting our choice of $B$ nilpotent.

Now suppose that $p=3$. We first show that $\bar{D}$ is generated by a 3 -cycle. Suppose that $D$ is not cyclic. Then $D$ contains an elementary abelian subgroup of order 9 , and in particular contains distinct elements $x$ and $y$ for which $x Z$ and $y Z$ is the product of $s$ and $t$ disjoint 3-cycles respectively (briefly, consider the centralizer of $g h$, for which $g h Z$ is the product of all the disjoint 3-cycles in $g Z$ and $h Z$. This has an elementary 
abelian subgroup contained in a conjugate of $D$ and containing elements whose images in $S_{n}$ are all the 3 -cycles making up $\left.g h Z\right)$. Then $C_{S_{n}}(x Z) \cong\left(\mathbb{Z}_{p}\left\langle S_{s}\right) \times S_{n-p s}\right.$ and $C_{S_{n}}(y Z) \cong\left(\mathbb{Z}_{p} 2 S_{t}\right) \times S_{n-p t}$. Hence $D$ contains elements $g$ and $h$ for which $g Z$ and $h Z$ are each a 3 -cycle and these 3 -cycles are disjoint. Write $R=\langle g, h\rangle \leq D$. We have $C_{S_{n}}(\bar{R}) \cong \bar{R} \times S_{n-6}$ and $C_{A_{n}}(\bar{R}) \cong \bar{R} \times A_{n-6}$. Now $\left[N_{\hat{S}_{n}}(R): C_{\hat{S}_{n}}(R)\right]=8$, and arguing as above we see that $\left[N_{\hat{A}_{n}}\left(R, b_{R}\right): C_{\hat{A}_{n}}(R)\right]$ is even for every block $b_{R}$ of $C_{\hat{A}_{n}}(R)$, a contradiction.

Hence $D$ is cyclic. Suppose that $|D|>3$. Then $D$ possesses an element $y$ of order 9. By an argument similar to above we may assume $y Z$ is a 9 -cycle. But then $y^{3} Z$ is a product of three distinct 3-cycles, which as we have seen cannot happen.

Hence $D$ has order three and is generated by an element $x$ where $x Z$ is a single 3 -cycle. We have $C_{\hat{A}_{n}}(D) \cong D \times \hat{A}_{n-3}$. The blocks of $C_{\hat{A}_{n}}(D)$ with defect group $D$ are in 1-1 correspondence with the blocks of defect zero of $\hat{A}_{n-3}$, and the action of $N_{\hat{A}_{n}}(D)$ on these blocks is given by the action of $\hat{S}_{n-3}$ on the blocks of defect zero of $\hat{A}_{n-3}$. Hence the nilpotent blocks of $\hat{A}_{n}$ with defect group $D$ are in 1-1 correspondence with orbits of length two of blocks of defect zero of $\hat{A}_{n-3}$ under the action of $\hat{S}_{n-3}$.

Now blocks of defect zero of $\hat{A}_{n-3}$ are covered by blocks of defect zero of $\hat{S}_{n-3}$. We consider faithful and non-faithful blocks separately. Note that $B$ is faithful if and only if the $B$-subpairs have kernel intersecting trivially with $Z$ (i.e., if and only if they correspond to faithful blocks of $\hat{A}_{n-3}$ ).

Suppose that $B$ is non-faithful. Blocks of defect zero correspond to 3-core partitions of $n-3$. By $[23,2.5 .7]$ irreducible characters of $S_{n-3}$ remain irreducible when restricted to $A_{n-3}$ if and only if the corresponding partition is not self-associate. Hence $\left[N_{\hat{A}_{n}}\left(D, b_{D}\right): C_{\hat{A}_{n}}(D)\right]=1$ if and only if the block of defect zero of $A_{n-3}$ corresponding to $b_{D}$ is labelled by a self-associate partition, and so the result follows in this case from Lemma 3.1.

Suppose that $B$ is faithful. Blocks of defect zero correspond to $\overline{3}$-core bar partitions of $n-3$. By [27, p.212] faithful irreducible characters of $\hat{S}_{n-3}$ remain irreducible when restricted to $\hat{A}_{n-3}$ if and only if the corresponding bar partition is odd. Hence $\left[N_{\hat{A}_{n}}\left(D, b_{D}\right): C_{\hat{A}_{n}}(D)\right]=1$ if and only if the block of defect zero of $\hat{A}_{n-3}$ corresponding to $b_{D}$ is labelled by an even $\overline{3}$-core bar partition, and so the result follows in this case from Lemma 3.2.

We have not yet considered all the perfect central extensions of $A_{6}$ and $A_{7}$. However, by the above theorem, neither yields a nilpotent 3-block with non-central defect group, and further it is easy to check that there are no nilpotent blocks of positive defect for the other odd primes.

It is appropriate here to extend our study to the double covers of the symmetric groups.

Proposition 3.4 Let $G=\hat{S}_{n}$ be the double cover of the symmetric group $S_{n}$ for $n \geq 5$ and let $p$ be an odd prime. If $p \geq 5$, then $G$ does not possess a nilpotent p-block of positive defect. If $p=3$, then every nilpotent block of positive defect is faithful. These have defect one, and occur if and only if there is a positive integer $m_{1}$ with $m_{1} \equiv 2,3 \bmod 4$ and $n=\left(3 m_{1}^{2}-m_{1}+6\right) / 2$ or a positive integer $m_{2}$ such that $m_{2} \equiv 1,2 \bmod 4$ and $n=\left(3 m_{2}^{2}+m_{2}+6\right) / 2$. 
Proof: Suppose first that $B$ is a non-faithful block of positive defect. By, for example, $[23,6.2 .2] l(B)=\sum p\left(w_{1}\right) \cdots p\left(w_{p-1}\right)$, where the sum runs over improper partitions $\left(w_{1}, \ldots, w_{p-1}\right)$ of the weight $w$ of $B$ and $p(x)$ is the number of partitions of $x$. But $p-1 \geq 2$ and $(w, 0, \ldots, 0)$ and $(0, w, 0, \ldots, 0)$ are improper partitions of $w$, so $l(B)>1$ and $B$ cannot be nilpotent.

Now suppose that $B$ is faithful of positive defect. Then by [28] $l(B)$ is at least $k((p-1) / 2, w)$, the number of $(p-1) / 2$-tuples of (possibly empty) partitions with sum $w$, where again $w$ is the weight of $B$. If $w \leq 2$, then $((w), \emptyset, \ldots, \emptyset)$ and $\left(\left(1^{w}\right), \emptyset, \ldots, \emptyset\right)$ are such $(p-1) / 2$-tuples of partitions, so $l(B)>1$ and $B$ cannot be nilpotent. Now suppose that $w=1$. If $p \geq 5$, then $(1, \emptyset, \ldots, \emptyset)$ and $(\emptyset, 1, \emptyset, \ldots, \emptyset)$ are such $(p-1) / 2$ tuples, and again $B$ cannot be nilpotent. We are left with the case $w=1$ and $p=3$. By $[28,13.17] l(B)=2$ if the $\overline{3}$-core $\mu$ of $B$ is even (in the sense that $n-3-r$ is even, where $r$ is the number of parts in $\mu$ ), and $l(B)=1$ if $\mu$ is odd. Note that since $B$ has cyclic defect groups, $B$ is nilpotent if and only if $l(B)=1$, and so the result follows from Lemma 3.2.

We now turn our attention to Puig's conjecture.

Lemma 3.5 Let $G=\hat{S}_{n}$ be the double cover of $S_{n}$, and let $B$ be a block of $G$ with defect group D. If $|D|>p^{2}$, then $l(B) \geq 3$.

Proof: Suppose first that $B$ is a non-faithful block. As above,

$$
l(B)=\sum p\left(w_{1}\right) \cdots p\left(w_{p-1}\right)
$$

where the sum runs over improper partitions $\left(w_{1}, \ldots, w_{p-1}\right)$ of the weight $w$ of $D$. If $|D|>p^{2}$, then $w \geq 2$. But $(w, 0, \ldots, 0),(0, w, 0, \ldots, 0)$ and $(w-1,1,0, \ldots, 0)$ are three such improper partitions, so $l(B) \geq 3$.

If $B$ is faithful, then by [28] $l(B)$ is at least $k((p-1) / 2, w)$, the number of $(p-1) / 2$ tuples of (possibly empty) partitions with sum $w$, where again $w$ is the weight of $B$. We have $w \geq 2$. Here $((w), \emptyset, \ldots, \emptyset),\left(\left(1^{w}\right), \emptyset, \ldots, \emptyset\right)$ and $((w-1,1), 1, \emptyset, \ldots, \emptyset)$ are three such tuples, so $l(B) \geq 3$.

Corollary 3.6 Let $B$ a p-block of $G$ for $p$ odd, where $G$ is quasisimple with $G / Z(G) \cong$ $A_{n}$ for some $n$. Then $B$ is nilpotent if and only if $l\left(b_{Q}\right)=1$ for every $B$-subgroup $\left(Q, b_{Q}\right)$.

Proof: If $B$ has abelian defect group $D$, then this is [30]. So we may assume $|D|>p^{2}$. Suppose $G \triangleleft H$, where $H \cong \hat{S}_{n}$, and let $B_{H} \in \operatorname{Blk}(H)$ covering $B$. Then $B_{H}$ has defect group $D$, and by Lemma $3.5 l\left(B_{H}\right) \geq 3$. But $l(B) \geq l\left(B_{H}\right) / 2>1$, so $B$ is not nilpotent, and of course we can take the $B$-subgroup $(1, B)$ to show the proposed equivalent condition is also not satisfied.

It remains to consider the exceptional covers, but in these cases it is easy to check that every block with non-central defect groups has more than one irreducible Brauer character. 


\section{$4 \quad$ Sporadic groups}

In this section we determine the nilpotent blocks with non-central defect groups of quasisimple groups $G$ where $G / Z(G)$ is one of the 26 sporadic simple groups. Note that due to Lemma 2.4 it suffices to consider the case $Z(G)$ is a $p^{\prime}$-group.

In order to provide a reasonably unified treatment of the classification of nilpotent blocks of the sporadic groups, we work from [20, Table 5.3]. However, in all cases the number of irreducible Brauer characters in the blocks are known, which would lead to a shorter but less illuminating proof. To avoid an overly long proof we do use these results in showing that Puig's conjecture holds.

We use [20, Table 5.3] and apply the following simple results to demonstrate the non-existence of such blocks in many cases:

Lemma 4.1 Suppose that $D$ is a defect group for a nilpotent block of a finite group $G$. Let $x \in D$ have order $p$ and write $Q=\langle x\rangle$ and $R=O_{p}\left(C_{G}(Q)\right)$. Then

(i) there is no p-regular $g \in N_{G}(Q)-C_{G}(Q)$ which fixes every block of $C_{G}(Q)$;

(ii) there is no p-regular $g \in N_{G}(R)-C_{G}(R)$ which fixes every block of $C_{G}(R)$.

Proof: Note that $R$ is contained in every defect group of every block of $C_{G}(Q)$. Hence $R \leq D$. The result then follows from the definition of a nilpotent block.

Lemma 4.2 Let $Q$ be a p-subgroup of $G$. If $\left|N_{G}(Q) / C_{G}(Q)\right|$ is prime to $p$ and, for every $n$, is strictly greater than the number of p-blocks of $C_{G}(Q)$ of dimension $n$ (or is greater than or equal to $n$ in the case $n$ is the dimension of the principal block of $\left.C_{G}(Q)\right)$, then $Q$ cannot be a subgroup of a defect group of a nilpotent block of $G$.

Proof: In this case every $p$-block of $C_{G}(Q)$ must be fixed by a $p$-regular element of $N_{G}(Q)-C_{G}(Q)$, and we apply Lemma 4.1 .

Lemma 4.3 Let $B$ be a nilpotent block with defect group $D$, and let $1 \neq Q \leq Z(D)$. Then $C_{G}(Q) / Q$ possesses a nilpotent block with defect group $D / Q$.

Proof: Let $b_{D} \in \operatorname{Blk}\left(D C_{G}(D)\right)$ with $b_{D}^{G}=B$. Now $D C_{G}(D) \leq C_{G}(Q)$, and $b=b_{D}^{C_{G}(Q)}$ is nilpotent. $D$ is the unique defect group of $b_{D}$ and $b^{G}=B$, so $D$ is a defect group of $b$. There is a one-to-one correspondence between the blocks of $C_{G}(Q)$ with defect group $D$ and the blocks of $C_{G}(Q) / Q$ with defect group $D / Q$. Let $\bar{b}$ be the correspondent of $b$. By Lemma $2.4 \bar{b}$ is nilpotent.

Write $Z=Z(G)$ and $\bar{G}=G / Z$. Note that when $Z$ is a $p^{\prime}$-group, for every $p$ subgroup $Q$ of $G$ we have $\overline{C_{G}(Q)}=C_{\bar{G}}(\bar{Q})$ and $\overline{N_{G}(Q)}=N_{\bar{G}}(\bar{Q})$.

Throughout our notation for the conjugacy classes of $\bar{G}$ follows that of [20].

Proposition 4.4 Let $G$ be a quasisimple group such that $\bar{G}$ is a sporadic simple group, with $|\bar{G}|_{p}=p$. Let $B$ be a p-block of maximal defect of $G$. Then $B$ is not nilpotent. 
Proof: Let $D \in \operatorname{Syl}_{p}(G)$. Note that $D$ is abelian. If $C_{\bar{G}}(\bar{D}) \leq \bar{D}$, then $C_{G}(D) \leq$ $D Z$ and every $p$-block of $C_{G}(D)$ is $N_{G}(D)$-stable. But $p$ does not divide $\left[N_{G}(D)\right.$ : $D C_{G}(D)$ ], whilst by Burnside's transfer theorem we cannot have $N_{G}(D)=C_{G}(D)$, so a block with defect group $D$ cannot be nilpotent. Hence, using [20, Table 5.3], we may rule out all but the following cases: $p=3$ and $\bar{G}=J_{1} ; p=5$ and $\bar{G}=M_{24}, J_{1}, J_{3}, J_{4}$; $p=7$ and $\bar{G}=M_{24}, J_{4}, C o_{3}, C o_{2}, S u z, L y, R u, F i_{22}, F i_{23}, H N ; p=11$ and $\bar{G}=C_{3}$, $C o_{1}, L y, F i_{22}, F i_{23}, F i_{24}^{\prime}, H N, F_{2} ; p=13$ and $\bar{G}=C o_{1}, R u, F i_{23}, F i_{24}^{\prime}, T h, F_{2}$; $p=17$ and $\bar{G}=F_{2}, F_{1} ; p=19$ and $G=F_{2}, F_{1} ; p=23$ and $\bar{G}=F_{2}, F_{1} ; p=29$ and $\bar{G}=F_{1} ; p=31$ and $\bar{G}=F_{1}$.

Applying Lemma 4.2 with $Q=D$ to these cases eliminates all but the case $p=3$ and $G=J_{1}$. Here $C_{\bar{G}}(\bar{D}) \cong D \times D_{10}$ and $N_{\bar{G}}(\bar{D}) \cong S_{3} \times D_{10}$, and it is clear that $N_{G}(D)$ fixes every block of $C_{G}(D)$.

Theorem 4.5 Let $B$ be a nilpotent $p$-block with non-central defect group $D$ of a quasisimple group $G$ such that $\bar{G}$ is a sporadic simple group. Then $|D|=3$ and $G$ is one of $M_{23}, J_{4}, L y$. In each of these cases $G$ does indeed possess a nilpotent block with defect group $D$.

Proof: We need only consider the case $p^{2}$ divides $|\bar{G}|$.

Suppose that $D$ is a non-central defect group of a nilpotent $p$-block $B$. We assume that $Z$ is a $p^{\prime}$-group. Choose $x \in D$ of order $p$, and write $Q=\langle x\rangle$. In each case $N_{\bar{G}}(\bar{Q})$ is given by [20, Table 5.3], and $C_{\bar{G}}(\bar{Q})$ may be deduced using [14].

Let $P=O_{p}\left(N_{G}(Q)\right)$. Then $P \leq D$.

We eliminate each possibility for the conjugacy class containing $x$ in turn using a succession of methods until we are left with the three cases listed. For each of these we then verify the existence of a nilpotent block with defect group $Q$.

Suppose that $N_{\bar{G}}(\bar{Q}) \cong \bar{H}_{1} \times \bar{H}_{2}$ and $C_{\bar{G}}(\bar{Q}) \cong \bar{Q} \times \bar{H}_{2}$ for some $H_{1}, H_{2}$ such that $Q \triangleleft H_{1}$, and $\bar{H}_{1} / \bar{Q}$ not a $p$-group. Then every $p$-block of $C_{G}(Q)$ is fixed by $N_{G}(Q)$ and $N_{G}(Q) / C_{G}(Q)$ is not a $p$-group, so $B$ cannot be nilpotent. In this way we eliminate the following pairs $(\bar{G}, C)$, where $C$ is the conjugacy class in $\bar{G}$ containing $x Z$ : $\left(M_{11}, 3 A\right)$, $\left(M_{12}, 3 B\right),\left(M_{24}, 3 B\right),\left(J_{2}, 3 B\right),\left(J_{2}, 5\right),\left(C_{3}, 3 C\right),\left(C o_{3}, 5 B\right),\left(C o_{2}, 3 B\right),\left(C_{2}, 5 B\right)$, $\left(C o_{1}, 3 D\right),(H S, 3 A),(H S, 5 B),(H e, 3 B),(H e, 7 A),(H e, 7 B),(R u, 5 B),\left(F i_{22}, 3 A\right)$, $\left(F i_{22}, 5 A\right),\left(F i_{23}, 3 A\right),\left(F i_{23}, 5 A\right),\left(F i_{24}^{\prime}, 7 A\right),\left(F_{2}, 3 A\right),\left(F_{2}, 5 A\right),\left(F_{1}, 3 C\right)$.

Suppose that $C_{\bar{G}}(\bar{P})$ is a $p$-group and $N_{G}(P) / C_{G}(P)$ is not a $p$-group. Then every p-block of $C_{G}(P)$ is $N_{G}(P)$-stable, and $B$ cannot be nilpotent. In this way we may eliminate the pairs $\left(M_{12}, 3 A\right),\left(J_{3}, 3 B\right),\left(J_{4}, 11\right),\left(C_{3}, 3 A\right),\left(C_{3}, 3 B\right),\left(C o_{3}, 5 A\right),\left(C o_{2}, 3 A\right)$, $\left(\mathrm{Co}_{2}, 5 \mathrm{~A}\right),\left(\mathrm{Co}_{1}, 3 \mathrm{C}\right),\left(\mathrm{Co}_{1}, 5 \mathrm{C}\right),(\mathrm{HS}, 5 \mathrm{~A}),(\mathrm{HS}, 5 \mathrm{C}),(\mathrm{McL}, 3),(\mathrm{McL}, 5),(\mathrm{Suz}, 3 \mathrm{~B})$, $(\mathrm{He}, 7 \mathrm{C}),(\mathrm{He}, 7 \mathrm{D}),(\mathrm{He}, 7 \mathrm{E}),(\mathrm{Ly}, 3 \mathrm{~B}),(\mathrm{Ly}, 5),(\mathrm{Ru}, 5 \mathrm{~A}),\left(\mathrm{O}^{\prime} \mathrm{N}, 7\right),\left(F i_{22}, 3 B\right),\left(F i_{22}, 3 C\right)$, $\left(F i_{22}, 3 D\right),\left(F i_{23}, 3 B\right),\left(F i_{23}, 3 C\right),\left(F i_{24}^{\prime}, 3 B\right)$ [since in this case no involution in $G$ centralizes a subgroup of the form $3^{1+10}$ ], $\left(F i_{24}^{\prime}, 3 C\right)$ [since in this case no involution in $\bar{G}$ centralizes a subgroup of the form $\left.C_{3}^{7}\right],\left(F i_{24}^{\prime}, 7 B\right),(H N, 3 B),(H N, 5 B),(H N, 5 C)$, $(H N, 5 D),(H N, 5 E),(T h, 3 B),(T h, 3 C),(T h, 5 A),\left(F_{2}, 3 B\right)$ [since no involution in $\bar{G}$ centralizes a subgroup of the form $\left.3^{1+8}\right],\left(F_{2}, 5 B\right),\left(F_{1}, 3 B\right),\left(F_{1}, 5 B\right),\left(F_{1}, 7 B\right)$, $\left(F_{1}, 13 B\right)$.

Suppose that $N_{\bar{G}}(\bar{Q}) \cong\left(\bar{H}_{1} \times \bar{H}_{2}\right) n$, where $n$ is an integer, and $C_{\bar{G}}(\bar{Q}) \leq \bar{H}_{1} \times \bar{H}_{2}$ for some $H_{1}$ and $H_{2}$ such that $\bar{Q}$ is a proper normal self-centralizing Sylow $p$-subgroup 
of $\bar{H}_{1}$. Then $H_{1} \leq N_{G}(Q)$ fixes every $p$-block of $C_{G}(Q)$ and $N_{G}(Q) / C_{G}(Q)$ is not a $p$-group. It follows that $B$ cannot be nilpotent. In this way we may eliminate the pairs $\left(C o_{1}, 5 A\right),\left(C o_{1}, 5 B\right),\left(C o_{1}, 7\right),(S u z, 3 C),(S u z, 5),(H e, 5 A),\left(O^{\prime} N, 3 A\right),\left(F i_{24}^{\prime}, 3 E\right)$, $\left(F i_{24}^{\prime}, 5 A\right),(H N, 5 A),(T h, 7 A),\left(F_{2}, 7 A\right),\left(F_{1}, 5 A\right),\left(F_{1}, 7 A\right),\left(F_{1}, 11 A\right),\left(F_{1}, 13 A\right)$.

Suppose that $N_{G}(Q) \cong\left(H_{1} \times H_{2}\right) n$ for some $H_{1}$ and $H_{2}$, where $n$ is an integer which is not a power of $p, Q \leq H_{1}$, and $H_{i} n$ (with the appropriate action) fixes every $p$-block of $H_{i}$ for $i=1,2$. Then $N_{G}(Q)$ fixes every $p$-block of $C_{G}(Q)$ and $N_{G}(Q) / C_{G}(Q)$ is not a $p$-group. It follows that $B$ cannot be nilpotent. In this way we may eliminate the pairs $\left(M_{22}, 3 A\right),\left(M_{24}, 3 A\right),\left(J_{2}, 3 A\right),\left(J_{3}, 3 A\right),\left(C o_{1}, 3 A\right),\left(C o_{1}, 3 B\right), \quad(S u z, 3 A), \quad(H e, 3 A)$, $(R u, 3 A),\left(F i_{24}^{\prime}, 3 A\right),(H N, 3 A),(T h, 3 A),\left(F_{1}, 3 A\right)$.

The only cases left unaccounted for are $F i_{23}$ and $F i_{24}^{\prime}$, where in each case $x Z$ belongs to the class labelled $3 D$. Suppose $\bar{G}=F i_{23}$ or $F i_{24}^{\prime}$ and $x Z \in 3 D$. In this case $Z=1$ (since $p=3$ ). We have already seen that a nilpotent 3-block of $G$ cannot contain elements of order three outside of $3 D$. Note that $x$ is conjugate to $x^{-1}$ (to see this consider the orders of the centralizers). Irreducible characters in such a block must vanish on $3 A, 3 B$ and $3 C$. This happens for only one irreducible character, and this lies in a block of defect zero.

If $\bar{G}=M_{23}$ and $p=3$, then $Z=1$ and $N_{G}(Q) \cong\left(\mathbb{Z}_{3} \times A_{5}\right) \cdot 2, C_{G}(Q) \cong \mathbb{Z}_{3} \times A_{5}$. Note that $M_{23}$ possesses just one 3-block of maximal defect, which cannot then be nilpotent. Hence we may assume $D=Q$, and so if $b_{Q}$ is a block of $C_{G}(Q)$ with $b_{Q}^{G}=B$, then $b_{Q}$ has defect group $Q$. Now $C_{G}(Q)$ has two blocks with defect group $Q$. The action of $N_{G}(Q)$ on the blocks of $C_{G}(Q)$ is given by the action of $S_{5}$ on the blocks of $A_{5}$, so the two blocks with defect group $Q$ are fused by $N_{G}(Q)$. Hence $\left[N_{G}\left(Q, b_{Q}\right): C_{G}(Q)\right]=1$, and $b_{Q}^{G}$ is nilpotent.

If $\bar{G}=J_{4}$ and $p=3$, then $Z=1$ and $N_{G}(Q) \cong\left(6 M_{22}\right) \cdot 2$ and $C_{G}(Q) \cong\left(6 M_{22}\right)$. By [14] $2 M_{22}$ possesses precisely two 3-blocks of defect zero fused by $2 M_{22} 2$ (the rest are fixed). These correspond to two 3-blocks of $C_{G}(Q)$ with defect group $Q$ fused by $N_{G}(Q)$. Hence $G$ possesses a nilpotent block with defect group $Q$ (the Brauer correspondent of the above blocks of $\left.C_{G}(Q)\right)$.

If $\bar{G}=L y$ and $x \in 3 A$, then $Z=1$ and $G$ possesses a nilpotent block with defect group $Q$, since $N_{G}(Q) \cong(3 M c L) \cdot 2, C_{G}(Q) \cong 3 M c L$, and $M c L$ possesses precisely two 3-blocks of defect zero which are fused in $M c L \cdot 2$ (all other 3-blocks of $M c L$ are fixed by $M c L$-2). These correspond to two 3-blocks of $C_{G}(Q)$ with defect group $Q$ fused by $N_{G}(Q)$. Hence $G$ possesses a nilpotent block with defect group $Q$ (the Brauer correspondent of the above blocks of $\left.C_{G}(Q)\right)$.

Note that we have shown in particular that whenever $p$ divides the Schur multiplier of a sporadic simple group, there is no nilpotent block of positive defect of the quotient group (by the Sylow $p$-subgroup of the centre).

We conclude:

Proposition 4.6 Let $G$ be a quasisimple group such that $G / Z(G)$ is a sporadic simple group and let $p$ be an odd prime. If $B$ is a nilpotent block of $G$, then $B$ has defect groups of order at most three.

We now address Puig's conjecture. 
Proposition 4.7 Let $G$ be a quasisimple group such that $G / Z(G)$ is a sporadic simple group and let $p$ be an odd prime. Let $B$ be a p-block of $G$. If $B$ has positive defect, then $l(B)>1$. In particular, $B$ is nilpotent if and only if $l\left(b_{Q}\right)=1$ for every $B$-subgroup $\left(Q, b_{Q}\right)$.

Proof: We may assume that $Z(G)$ is a $p^{\prime}$-group. Let $D$ be a defect group of $B$. If $D$ is cyclic, then the result follows from the theory of blocks with cyclic defect groups. In the following table we list all the numbers of irreducible Brauer characters in blocks with non-cyclic defect groups, along with a reference. A '*' will be used to denote a faithful block in a group with non-trivial centre. The result then follows from examination of the table.

\begin{tabular}{|l|l|l|l|}
\hline$G / Z(G)$ & $|D|$ & $\ell(B)$ & reference \\
\hline$M_{11}$ & $3^{2}$ & 7 & {$[18]$} \\
$M_{12}$ & $3^{3} / 3^{3}$ & $8 / 8^{*}$ & {$[18]$} \\
$M_{22}$ & $3^{2} / 3^{2} / 3^{2}$ & $5 / 5^{*} / 5^{*}$ & {$[18]$} \\
$M_{23}$ & $3^{2}$ & 7 & {$[18]$} \\
$M_{24}$ & $3^{3}$ & 7 & {$[18]$} \\
$J_{2}$ & $3^{3} / 3^{3} / 5^{2} / 5^{2}$ & $8 / 8^{*} / 6 / 6^{*}$ & {$[18]$} \\
$J_{3}$ & $3^{5}$ & 8 & {$[18]$} \\
$J_{4}$ & $3^{3} / 3^{3} / 3^{2} / 11^{3}$ & $9 / 9 / 5 / 40$ & {$[8] /[10]$} \\
$H S$ & $3^{2} / 3^{2} / 3^{2} / 5^{3} / 5^{3}$ & $7 / 7 / 5^{*} / 10 / 10^{*}$ & {$[18]$} \\
$M c L$ & $3^{6} / 5^{3} / 5^{3}$ & $10 / 12 / 12^{*}$ & {$[18]$} \\
$S u z$ & $3^{7} / 3^{2} / 3^{7}$ & $13 / 5 / 10^{*}$ & {$[18]$} \\
$L y$ & $3^{7} / 5^{6}$ & $21 / 35$ & \\
$H e$ & $3^{3} / 3^{2} / 5^{2} / 7^{3}$ & $7 / 7 / 14 / 10$ & {$[18]$} \\
$R u$ & $3^{3} / 3^{3} / 5^{3} / 5^{3}$ & $9 / 9^{*} / 18 / 18^{*}$ & {$[18]$} \\
$O^{\prime} N$ & $3^{4} / 3^{2} / 7^{3} / 7^{3}$ & $14 / 5 / 19 / 19^{*}$ & {$[18]$} \\
$C o_{3}$ & $3^{7} / 5^{3}$ & $20 / 18$ & {$[18]$} \\
$C o_{2}$ & $3^{6} / 5^{3}$ & $23 / 16$ & {$[18]$} \\
$C o_{1}$ & $3^{9} / 3^{3} / 3^{2} / 5^{4} / 5^{2} / 7^{2}$ & $29 / 7 / 5 / 29 / 12 / 21$ & {$[7]$} \\
$F i_{22}$ & $3^{9} / 3^{9} / 5^{2} / 5^{2} / 5^{2} / 5^{2}$ & $22 / 18^{*} / 16 / 16^{*} / 16^{*} / 16^{*}$ & {$[18]$} \\
$F i_{23}$ & $3^{13} / 5^{2} / 5^{2}$ & $32 / 16 / 16$ & {$[5]$} \\
$F i_{24}^{\prime}$ & $3^{16} / 3^{2} / 5^{2} / 5^{2} / 5^{2} / 5^{2} / 5^{2}$ & $25 / 4 / 16 / 16 / 14 / 16^{*} / 16^{*}$ & {$[4]$} \\
$T h$ & $5^{2} / 5^{2} / 7^{3} / 7^{3} / 7^{3}$ & $14^{*} / 14^{*} / 22 / 22^{*} / 22^{*}$ & {$[4]$} \\
$H N$ & $3^{10} / 5^{3} / 7^{2}$ & $10 / 30 / 24$ & {$[6]$} \\
$F_{2}=B$ & $3^{6} / 3^{2} / 5^{6}$ & $20 / 7 / 16$ & {$[9]$} \\
$3_{1}=M$ & $3^{6} / 3^{2} / 3^{2} / 3^{2} / 3^{13}$ & $71 / 7 / 7 / 5 / 31^{*}$ & {$[18]$} \\
\hline & $3^{20} / 3^{3} / 5^{9} / 5^{2} / 7^{6} / 7^{2} / 11^{2} / 13^{3}$ & $83 / 7 / 91 / 16 / 70 / 24 / 45 / 52$ & \\
\hline
\end{tabular}

Table 1: Numbers of irreducible Brauer characters in blocks with non-cyclic, noncentral defect groups of sporadic groups 
If $G=L y$ and $p=3$, then by [14] $G$ has thirty 3-regular conjugacy classes. By [31], aside from the principal block, $G$ has five 3-blocks of defect zero and two 3-blocks of defect one. Since we have shown that neither of these blocks of defect one is nilpotent, it follows that they each have two irreducible Brauer characters. Hence the principal block has 21 irreducible Brauer characters for $p=3$. A similar computation for $p=5$ shows that the principal 5-block of $L y$ has 35 irreducible Brauer characters (and this is the unique 5-block with non-cyclic defect groups).

If $G=T h$ and $p=3$, then by [14] $G$ has sixteen 3-regular conjugacy classes. By [32] $G$ has four 3-blocks of defect zero and one 3-block of defect one (which we have seen cannot be nilpotent, so has two irreducible Brauer characters. Hence the principal block 3-possesses ten irreducible Brauer characters. A similar computation for $p=5$ shows that the principal 5-block of $T h$ has 30 irreducible Brauer characters (and this is the unique 5-block with non-cyclic defect groups). For $p=7$, by [32] $G$ has fourteen blocks of defect zero, a block of defect one (with six irreducible Brauer characters, by consideration of the inertial quotient) and the principal block, which must then have 24 irreducible Brauer characters.

If $G / Z(G)=F i_{24}^{\prime}$, then the result follows from [4] when $p=3$, and when $p=5$ or 7 for non-faithful blocks in the case $|Z(G)|=3$. Suppose $|Z(G)|=3$, and consider faithful blocks $B$ with a defect group $D$ covering a block, say $c$ of $Z(G)$. Suppose first $p=5$. We have $D=5^{2}$ and from $[4, \mathrm{p} .141] k(B)=20$. Note that $G$ has only one conjugacy class of elements of order 5. If $x \in D \backslash\{1\}$ and $b \in \operatorname{Blk}\left(C_{G}(x)\right)$ with $b^{G}=B$, then $C_{G}(x)=3 \times 5 \times A_{9}$ and $b=c \times B_{0}(5) \times b^{\prime}$ for some $b^{\prime} \in \operatorname{Blk}\left(A_{9}\right)$ with $D\left(b^{\prime}\right)=5$. As shown in [4, p.114] $A_{9}$ has three such blocks $b_{0}^{\prime}=B_{0}\left(A_{9}\right), b_{1}^{\prime}, b_{2}^{\prime}$ and $l\left(b_{0}^{\prime}\right)=l\left(b_{1}^{\prime}\right)=4$, $l\left(b_{2}^{\prime}\right)=2$. The canonical characters of the root blocks of $b_{2}^{\prime}$ and $b_{1}^{\prime}$ are linear and degree 3 characters of $C_{A_{9}}(5)=5 \times A_{4}$, respectively. Since $N_{G}(D)=3 .\left(5^{2}: 4 A_{4} \times A_{4}\right) .2$ and a Sylow 3-subgroup of $N_{G}(D)$ is isomorphic to $3_{+}^{1+2}$, it follows that $c \times B_{0}(5) \times b_{0}^{\prime}$ and $c \times B_{0}(5) \times b_{2}^{\prime}$ induce the same block $B$ of $G$ and so $l(B)=20-4-2=14$. Also $c \times B_{0}(5) \times b_{1}^{\prime}$ induces another block $B$ of $G$ and $l(B)=20-4=16$. If $p=7$, then by $[4, \mathrm{p} .141], k(B)=k\left(B_{0}\left(F i_{24}^{\prime}\right)\right)$ and $C_{G}(x)=3 \times C_{F i_{24}^{\prime}}(x)$ for any $x \in D \backslash\{1\}$. Thus $l(B)=l\left(B_{0}\left(F i_{24}\right)\right)=22$.

\section{$5 \quad$ Notation for classical groups and their blocks}

Let $V$ be a linear, unitary, non-degenerate orthogonal or symplectic space over the field $\mathbb{F}_{q}$, where $q=r^{a}$ for some prime $r \neq p$. We will follow the notation of [3], [11], [16] and [17].

If $V$ is orthogonal (and $q$ is odd), then there is a choice of equivalence classes of quadratic forms. Write $\eta(V)$ for the type of $V$ as defined in [17], so $\eta(V)=\eta=+$ or -. Write $\eta(V)=+$ if $V$ is linear and $\eta(V)=-$ if $V$ is unitary. If $V$ is non-degenerate orthogonal or symplectic, then denote by $I(V)$ the group of isometries on $V$ and let $I_{0}(V)=I(V) \cap \mathrm{SL}(V)$.

If $V$ is symplectic, then $I(V)=I_{0}(V)=\operatorname{Sp}_{2 n}(q)$.

If $V$ is a $(2 n+1)$-dimensional orthogonal space, then $I(V)=\left\langle-1_{V}\right\rangle \times I_{0}(V)$ with $I_{0}(V)=\mathrm{SO}_{2 n+1}(q)$. 
If $V$ is a $2 n$-dimensional orthogonal space, then $I(V)=O^{\eta}(V)=O_{2 n}^{\eta}(q)$ and $I_{0}(V)=\mathrm{SO}_{2 n}^{\eta}(q)$.

If $V$ is a $2 n$-dimensional non-degenerate orthogonal or symplectic space, then denote by $J_{0}(V)$ the conformal isometries of $V$ with square determinant. If $V$ is orthogonal of dimensional at least two, then write $D_{0}(V)$ for the special Clifford group of $V$ (cf. [17]).

Denote by $\mathrm{GL}^{+}(V)$ the general linear group $\mathrm{GL}(V)$ and $\mathrm{GL}^{-}(V)$ the unitary group $\mathrm{U}(V)$.

Let $G=\mathrm{GL}^{\eta}(V)$ or $I(V)$. Write $\mathcal{F}_{q}=\mathcal{F}_{q}(G)$ for the set of polynomials (with coefficients in $\mathbb{F}_{q}$ ) serving as elementary divisors for semisimple elements of $G$ (cf. [3, p.6]). For $\Gamma \in \mathcal{F}_{q}(G)$, let $d_{\Gamma}$ be the degree of $\Gamma$, and $\delta_{\Gamma}$ be the reduced degree defined as in [3], [16] and [17]. So $\delta_{\Gamma}=d_{\Gamma}$ or $\delta_{\Gamma}=\frac{1}{2} d_{\Gamma}$ according as $d_{\Gamma}$ is even or odd (note that if $V$ is symplectic or orthogonal, $\Gamma$ must have even degree unless $\Gamma=X \pm 1$ ).

If $G=\mathrm{GL}(V)$, then let $\epsilon_{\Gamma}=1$. Otherwise $\epsilon_{\Gamma}$ is given by [3, p.6]. Let $e_{\Gamma}$ be the multiplicative order of $\epsilon_{\Gamma} q^{\delta_{\Gamma}}$ modulo $p$. Thus we may write $e_{\Gamma} \delta_{\Gamma}=e p^{\alpha_{\Gamma}} \delta_{\Gamma}^{\prime}$ for some $\alpha_{\Gamma}$ and $\delta_{\Gamma}^{\prime}$ with $p \nmid \delta_{\Gamma}^{\prime}$, where $e=e_{X-1}$.

Given a semisimple element $s \in G$, there is a unique orthogonal decomposition $V=\sum_{\Gamma \in \mathcal{F}_{q}} V_{\Gamma}(s)$, with $s=\prod_{\Gamma \in \mathcal{F}_{q}} s(\Gamma)$, where the $V_{\Gamma}(s)$ are nondegenerate subspaces of $V$ and $s(\Gamma) \in \mathrm{GL}\left(V_{\Gamma}(s)\right), \mathrm{U}\left(V_{\Gamma}(s)\right)$ or $I\left(V_{\Gamma}(s)\right)$ (depending on $G$ ) has minimal polynomial $\Gamma$. This is called the primary decomposition of $s$. Write $m_{\Gamma}(s)$ for the multiplicity of $\Gamma$ in $s(\Gamma)$. We have $C_{G}(s)=\prod_{\Gamma \in \mathcal{F}_{q}} C_{\Gamma}(s)$, where $C_{\Gamma}(s)=I\left(V_{\Gamma}(s)\right)$ or $\mathrm{GL}^{\epsilon_{\Gamma}}\left(m_{\Gamma}(s), q^{\delta_{\Gamma}}\right)$ as appropriate.

\section{Blocks of linear and unitary groups}

Suppose $G=\mathrm{GL}_{n}^{\eta}(q)=\mathrm{GL}^{\eta}(V)$ and $p$ is odd and distinct to $r$, and let $B$ be a $p$-block of $G$ with a defect group $D$ and label $(s, \kappa)$. Then we may write

$$
V=V_{0} \perp V_{+}, \quad D=D_{0} \times D_{+}, \quad s=s_{0} \times s_{+},
$$

where $V_{0}=C_{V}(D), V_{+}=[D, V], s_{0} \in G_{0}=\mathrm{GL}^{\eta}\left(V_{0}\right)$ and $s_{+} \in G_{+}:=\mathrm{GL}^{\eta}\left(V_{+}\right)$. For convenience we denote $\mathrm{GL}^{\eta}(V)$ by $G(V)$ and $\mathrm{SL}^{\eta}(V)$ by $S(V)$.

Theorem 6.1 Let $G=\mathrm{GL}^{\eta}(V)=\mathrm{GL}^{\eta}(n, q)$ and suppose $p$ is odd with $p \nmid q$. Then the following are equivalent.

(a) $B$ is a nilpotent block of $G$.

(b) $m_{\Gamma}\left(s_{+}\right)=e_{\Gamma}=1$ for all $\Gamma \in \mathcal{F}_{q}$ which are elementary divisors of $s_{+}$.

(c) $\kappa_{\Gamma}$ is a $e_{\Gamma}$-core of $m_{\Gamma}(s)$ whenever $e \nmid \delta_{\Gamma}$, and $m_{\Gamma}(s) \leq 1$ whenever $e \mid \delta_{\Gamma}$, where $\kappa_{\Gamma}=\emptyset$ is viewed as an $e_{\Gamma}$-core of $0=m_{\Gamma}(s)$.

(d) Let $\left(D, b_{D}\right)$ be a Sylow B-subgroup and $\theta$ the canonical character of $b_{D}$. Then $C_{G}(D)=G_{0} \times C_{+}$and $\theta=\theta_{0} \times \theta_{+}$, where $C_{+}:=C_{G_{+}}\left(D_{+}\right)$is regular in $G_{+}, \theta_{0}$ is an irreducible character of defect 0 of $G_{0}$ labelled by $\left(s_{0}, \kappa\right)$ and $\theta_{+}= \pm R_{T_{+}}^{C_{+}}\left(s_{+}\right)$with $T_{+}=C_{C_{+}}\left(s_{+}\right)$a torus of both $G_{+}$and $C_{+}$, and $D_{+}=O_{p}\left(T_{+}\right)$. Here $R_{T_{+}}^{C_{+}}\left(s_{+}\right)$is the Deligne-Lusztig generalized character.

In particular, if $B$ is nilpotent, then $D$ is abelian. 
Proof: Let $s_{+}=\prod_{\Gamma} s(\Gamma)$ be a primary decomposition, so that $V_{+}=\bigoplus_{\Gamma} V_{\Gamma}$ with $V_{\Gamma}$ the underlying space of $s(\Gamma)$. Write $m_{\Gamma}$ for $m_{\Gamma}\left(s_{+}\right)$. Then

$$
C_{G_{+}}\left(s_{+}\right)=\prod_{\Gamma} C_{\Gamma}
$$

where $C_{\Gamma} \cong \mathrm{GL}^{\epsilon_{\Gamma}}\left(m_{\Gamma}, q^{\delta_{\Gamma}}\right)$. We may suppose $D_{+} \in \operatorname{Syl}_{p}\left(C_{G_{+}}\left(s_{+}\right)\right)$, so that

$$
D_{+}=\prod_{\Gamma} D_{\Gamma}, \quad D_{\Gamma} \in \operatorname{Syl}_{p}\left(C_{\Gamma}\right) .
$$

So $D$ is a direct product of wreath product $p$-groups.

Let $\Gamma$ be an elementary divisor of $s_{+}$. Since $C_{V_{\Gamma}}\left(D_{\Gamma}\right)=0$, it follows that $p$ divides $q^{\delta_{\Gamma} m_{\Gamma}}-\epsilon_{\Gamma}$ and so $e_{\Gamma} \mid m_{\Gamma}$. Hence we may write $m_{\Gamma}=e_{\Gamma} w_{\Gamma}$ for some $w_{\Gamma}$. Let $A(D)$ be the subgroup of $D$ generated by all the abelian normal subgroups of $D$. By $[1$, Theorem 2], $A(D)$ is the base subgroup of $D$. Write $R=A(D)$. Then

$$
R=D_{0} \times \prod_{\Gamma}\left(R_{\Gamma}\right)^{w_{\Gamma}}, \quad C_{G}(R)=G_{0} \times \prod_{\Gamma}\left(K_{\Gamma}\right)^{w_{\Gamma}}, \quad K_{\Gamma} \cong \mathrm{GL}^{\epsilon}\left(\delta_{\Gamma}^{\prime}, q^{e p^{\alpha_{\Gamma}}}\right)
$$

where $\epsilon=\epsilon_{X-1}$ and $R_{\Gamma}=O_{p}\left(Z\left(K_{\Gamma}\right)\right)$ is cyclic and $\left(R_{\Gamma}\right)^{w_{\Gamma}}$ is a diagonal subgroup of $\mathrm{GL}^{\epsilon_{\Gamma}}\left(w_{\Gamma}, q^{\delta_{\Gamma} e_{\Gamma}}\right) \leq C_{\Gamma}$. Thus $C_{G_{\Gamma}}\left(\left(R_{\Gamma}\right)^{w_{\Gamma}}\right)=\left(K_{\Gamma}\right)^{w_{\Gamma}}, C_{G}(R)$ is regular in $G$,

$$
N_{G_{\Gamma}}\left(\left(R_{\Gamma}\right)^{w_{\Gamma}}\right)=K_{\Gamma}\left\langle\mathbf{S}\left(w_{\Gamma}\right),\right.
$$

and we may suppose $s \in C_{G}(R)$, where $G_{\Gamma}:=G\left(V_{\Gamma}\right)$ and $\mathbf{S}(m)$ is the symmetric group on $m$ letters.

Suppose $w_{\Delta} \geq 2$ for some $\Delta$. Then there is $P\left(D_{\Delta}\right) \leq\left(R_{\Delta}\right)^{w_{\Delta}}$ such that

$$
C_{G_{\Delta}}\left(P\left(D_{\Delta}\right)\right)=\left(K_{\Delta}\right)^{w_{\Delta}-2} \times \mathrm{GL}^{\epsilon}\left(2 \delta_{\Delta}^{\prime}, q^{e p^{\alpha} \Delta}\right) \quad \text { and } \quad P\left(D_{\Delta}\right)=O_{p}\left(C_{G_{\Delta}}\left(P\left(D_{\Delta}\right)\right)\right) .
$$

Thus $C_{C_{\Delta}}\left(P\left(D_{\Delta}\right)\right)=\mathrm{GL}^{\epsilon_{\Delta}}\left(1, q^{\delta_{\Delta} e_{\Delta}}\right)^{w_{\Delta}-2} \times \mathrm{GL}^{\epsilon_{\Delta}}\left(2, q^{\delta_{\Delta} e_{\Delta}}\right)$ and

$$
N_{C_{G_{\Delta}}\left(P\left(D_{\Delta}\right)\right)}\left(\left(R_{\Delta}\right)^{w_{\Delta}}\right)=\left(K_{\Delta}\right)^{w_{\Delta}-2} \times K_{\Delta} \prec \mathbf{S}(2) .
$$

There is an element $y_{\Delta}$ of $\left(N_{C_{\Delta}}\left(\left(R_{\Delta}\right)^{w_{\Delta}}\right) \cap C_{G_{\Delta}}\left(P\left(D_{\Delta}\right)\right)\right) \backslash C_{C_{\Delta}}\left(\left(R_{\Delta}\right)^{w_{\Delta}}\right)$ which swaps exactly two factors $K_{\Delta}$ in $C_{G_{\Delta}}\left(\left(R_{\Delta}\right)^{w_{\Delta}}\right),\left|y_{\Delta}\right|=4, y_{\Delta}^{2} \in C_{C_{\Delta}}\left(\left(R_{\Delta}\right)^{w_{\Delta}}\right)$ and $\operatorname{det}\left(y_{\Delta}\right)=1$.

Writing $y_{\Gamma}:=1$ when $\Gamma \neq \Delta$, define

$$
y:=1_{V_{0}} \times \prod_{\Gamma} y_{\Gamma} \quad \text { and } \quad P(D):=D_{0} \times \prod_{\Gamma \neq \Delta}\left(R_{\Gamma}\right)^{w_{\Gamma}} \times P\left(D_{\Delta}\right) .
$$

Then $y \in\left(N_{C_{G}(P(D))}(R) \cap C_{G}(s)\right) \backslash C_{G}(R)$ and $y^{2} \in C_{G}(R)$. Let $\left(R, b_{R}\right)$ be a $B$-subgroup, so that $D\left(b_{R}\right)=R$ and we may suppose $b_{R} \subseteq \mathcal{E}_{p}\left(C_{G}(R),(s)\right)$. Since $y \in C_{G}(s)$, it follows that $y \in N_{G}\left(R, b_{R}\right) \backslash C_{G}(R)$, so $B$ is not nilpotent, a contradiction. Thus $m_{\Gamma}=e_{\Gamma}$ for all $\Gamma$ and $D$ is abelian with each $D_{\Gamma}$ cyclic.

For each $\Gamma$,

$$
N_{\mathrm{GL}^{\epsilon_{\Gamma}\left(e_{\Gamma}, q^{\delta} \Gamma\right)}}\left(D_{\Gamma}\right)=\left\langle\tau_{\Gamma}, C_{\mathrm{GL}^{\epsilon_{\Gamma}\left(e_{\Gamma}, q^{\delta_{\Gamma}}\right)}}\left(D_{\Gamma}\right)\right\rangle, \quad C_{\mathrm{GL}^{\epsilon_{\Gamma}\left(e_{\Gamma}, q^{\left.\delta_{\Gamma}\right)}\right.}}\left(D_{\Gamma}\right)=\mathrm{GL}^{\epsilon_{\Gamma}}\left(1, q^{e_{\Gamma} \delta_{\Gamma}}\right),
$$


where $\tau_{\Gamma} \in \mathrm{GL}^{\epsilon_{\Gamma}}\left(e_{\Gamma}, q^{\delta_{\Gamma}}\right)$ has order $e_{\Gamma}$ modulo $\mathrm{GL}^{\epsilon_{\Gamma}}\left(1, q^{e_{\Gamma} \delta_{\Gamma}}\right)$. If $\tau=1_{V_{0}} \times \prod_{\Gamma} \tau_{\Gamma}$, then $\tau \in N_{G}(D) \cap C_{G}(s)$ and so $\tau \in N_{G}\left(D, b_{D}\right)$, where $b_{D}$ is the block of $C_{G}(D)$ labelled by $(s, \kappa)$.

Since $e_{\Gamma}$ and $p$ are coprime, it follows that $\tau$ is a $p^{\prime}$-element, $e_{\Gamma}=1$ and $C_{\Gamma}=$ $\mathrm{GL}^{\epsilon_{\Gamma}}\left(1, q^{\delta_{\Gamma}}\right)$. In particular, $C_{G_{+}}\left(s_{+}\right)$is a torus and $e \mid \delta_{\Gamma}$.

Conversely, if $m_{\Gamma}\left(s_{+}\right)=e_{\Gamma}=1$, then $C_{\Gamma}=\operatorname{GL}^{\epsilon_{\Gamma}}\left(1, q^{\delta_{\Gamma}}\right)$ and so $N_{C_{\Gamma}}\left(D_{\Gamma}\right)=$ $C_{C_{\Gamma}}\left(D_{\Gamma}\right)=C_{\Gamma}$. Thus $D$ is abelian,

$$
N_{C_{G}(s)}(D)=C_{C_{G}(s)}(D)=C_{G}(s)
$$

and $N_{G}\left(D, b_{D}\right)=C_{G}(D)$. By Proposition 2.1, $B$ is nilpotent.

Note that $e_{\Gamma}=1$ if and only if $e \mid \delta_{\Gamma}$, so since $D$ is a Sylow $p$-subgroup of $C_{G}(s)$, it follows that $m_{\Gamma}\left(s_{0}\right)=0$ whenever $m_{\Gamma}\left(s_{+}\right)=1$, so that $m_{\Gamma}(s)=1$. If $m_{\Gamma}\left(s_{0}\right)=m_{\Gamma}(s) \neq 0$, then by definition, $\kappa_{\Gamma}$ is an $e_{\Gamma^{-}}$core of $m_{\Gamma}(s)$. The equivalences of (b), (c) and (d) now follow by [11] and [16].

For integers $c$ and $m$, we write $p^{c} \| m$ when $p^{c} \mid m$ and $p^{c+1} \nmid m$.

Remark 6.2 In the notation of the proof above, we may suppose the element $\tau \in$ $C_{G_{+}}\left(s_{+}\right)$has determinant 1 whenever $e_{\Gamma} \geq 2$ for some $\Gamma$.

Proof We may suppose $q=q^{\delta_{\Gamma}}$, so that $e_{\Gamma}=e$. Let $\mathbf{T}$ be the diagonal maximal torus of $\mathbf{G}=\mathrm{GL}(\mathbb{F} \otimes V), \sigma$ the Frobenius map of $\mathbf{G}$ such that $C_{\mathbf{G}}(\sigma)=\mathrm{GL}^{\epsilon}(e, q)$, where $\mathbb{F}$ is the algebraic closure of $\mathbb{F}_{q}$.

Choose matrices $P_{i j}$ (with $i \neq j$ ) of $\mathbf{G}$ such that $P_{i j}$ acts on $\mathbf{T}$ as the permutation swapping the $(i, i)$ and $(j, j)$ entries of $\mathbf{T}$, the entries of $P_{i j}$ are 1 or $-1, \operatorname{det}\left(P_{i j}\right)=1$ and $P_{i j}$ is fixed by $\sigma$. If $W$ is generated by the matrices $P_{i j}$, then $N_{\mathbf{G}}(\mathbf{T})=\mathbf{T} W$ and $W \mathbf{T} / \mathbf{T} \cong \mathbf{S}(e)$.

Note $\omega^{\sigma}=\omega$ for each $\omega \in W$. Let $\omega_{0} \in W$ such that

$$
C_{\mathbf{T}}\left(\sigma \omega_{0}\right) \cong \mathrm{GL}^{\epsilon}\left(1, q^{e}\right),
$$

so that $\omega_{0}$ acts on $\mathbf{T}$ as the cycle $(1,2, \cdots, e)$. Now $C_{\mathbf{T}}\left(\sigma \omega_{0}\right)$ is conjugate in $\mathbf{G}$ to the Coxeter torus $\mathrm{GL}^{\epsilon}\left(1, q^{e}\right)$ of $C_{\mathbf{G}}(\sigma)$ and $\omega_{0}$ normalizes $C_{\mathbf{T}}\left(\sigma \omega_{0}\right)$. Thus there is an element $\beta \in \mathrm{SL}^{\eta}(e, q)$ such that $\beta$ normalizes the torus $\mathrm{GL}^{\epsilon}\left(1, q^{e}\right)$ and $\beta$ has order $e$ modulo $\mathrm{GL}^{\epsilon}\left(1, q^{e}\right)$. Since $N_{\mathrm{GL}^{\epsilon}(e, q)}\left(\mathrm{GL}^{\epsilon}\left(1, q^{e}\right)\right) / \mathrm{GL}^{\epsilon}\left(1, q^{e}\right) \cong \mathbb{Z}_{e}$, it follows that

$$
N_{\mathrm{GL}^{\epsilon}(e, q)}\left(\mathrm{GL}^{\epsilon}\left(1, q^{e}\right)\right)=\left\langle\beta, \mathrm{GL}^{\epsilon}\left(1, q^{e}\right)\right\rangle
$$

and we may suppose $\tau=\beta$.

\section{$7 \quad$ A set of technical conditions}

In order to investigate nilpotent blocks of exceptional groups of Lie type it is not sufficient just to find the nilpotent blocks of classical groups. We need in addition some somewhat technical properties which we will identify in classical groups and their 
extensions by diagonal automorphisms which relate to nilpotency (in particular, they will be used to examine centralizers of elements of defect groups of nilpotent blocks).

These properties also ensure that Puig's conjecture holds for the groups under consideration.

We state these properties in this section, along with some general results which will be needed in proving that they hold for classical groups.

Let $G$ be a finite group, $Q$ a $p$-subgroup of $G$, and $B \in \operatorname{Blk}(G)$. If $p$ is odd, we denote by $A(Q)$ the subgroup of $Q$ generated by all the abelian normal subgroups of $Q$. Recall that a $B$-subgroup $\left(R, b_{R}\right)$ is called self-centralizing if $Z(R)$ is a defect group of $b_{R} \in \operatorname{Blk}\left(C_{G}(R)\right)$.

We will prove for some finite groups of Lie type that one of (a)-(d) of the following holds. A feature of these properties is that none can be satisfied by a nilpotent block with non-abelian defect groups.

Property 7.1 Let $K$ be a normal subgroup of a finite group $H$, and let $B \in \operatorname{Blk}(K)$ and $B_{H} \in \operatorname{Blk}(H)$ such that $B_{H}$ covers $B$.

(a) There exist B-subgroups $(P, g) \leq(R, b)$, where $R$ is abelian, with abelian defect groups $D(g)$ and $D(b)$ respectively such that $D(g)=D(b)$, and an element $y \in$ $N_{C_{K}(P)}(R)$ such that $y^{4}=1,[y, x] \notin Z(K)$ for some $x \in R$ and $y^{2} \in C_{K}(R)$, and such that $\theta^{y}=\theta$, where $\theta$ is the canonical character of $b$. There exist $B_{H^{-}}$ subgroups $\left(P, g_{H}\right) \leq\left(R, b_{H}\right)$ where $g_{H}$ covers $g$, $b_{H}$ covers $b$, such that $b_{H}^{y}=b_{H}$ and $D\left(b_{H}\right)=D\left(g_{H}\right)$ is abelian for defect groups $D\left(b_{H}\right)$ and $D\left(g_{H}\right)$ of $b_{H}$ and $g_{H}$ respectively.

(a*) Property (a) above holds, and there exist subgroups $N_{i} \triangleleft M_{i}$ of $H$, and characters $\theta_{i} \in \operatorname{Irr}\left(N_{i}\right)$ for $i=1,2$ such that $M_{i} / N_{i}$ is abelian,

$$
Z(K) \leq N_{1} \times N_{2} \leq C_{K}(R) \leq C_{H}(R) \leq M_{1} \circ M_{2},
$$

$\theta$ covers $\theta_{1} \times \theta_{2}, Z_{0} \cap N_{2}=1, \theta_{2}$ has a $y$-stable extension to $M_{2}$ and $[y, x]=1$ or $[y, x] \in Z_{0} N_{2}$ according as $x \in M_{1}$ or $M_{2}$, where $Z_{0} \leq Z\left(M_{1}\right) \cap Z\left(M_{2}\right)$ such that $M_{1} \circ M_{2}$ is a central product over $Z_{0}$

(b) $D(B) \cong 3_{+}^{1+2}, \ell(B) \geq 2$, and either $D\left(B_{H}\right)=D(B)$ or $D\left(B_{H}\right) \cong \mathbb{Z}_{3} \succ \mathbb{Z}_{3}$.

(c) $D(B)=3^{2}, \ell(B) \geq 2$, and either $D\left(B_{H}\right)=D(B)$ or $D\left(B_{H}\right) \cong 3_{+}^{1+2}$.

(d) Both $D(B)$ and $D\left(B_{H}\right)$ are abelian.

Remark $7.2(i)$ Suppose that $(P, g) \leq(R, b)$ are $B$-subgroups with abelian defect groups $D(g)$ and $D(b)$, and $R$ is abelian. By [15, Lemma 4.1], there exists a $B_{H^{-}}$ subgroup $\left(P, g_{H}\right)$ such that $g_{H}$ covers $g$. Since $R \leq D(b)$ is abelian and $(P, g) \leq(R, b)$, it follows that $(R, b)$ is a $g$-subgroup and by [15, Lemma 4.1] again, there exists a $g_{H^{-}}$ subgroup $\left(R, b_{H}\right)$ such that $b_{H}$ covers b. Thus $\left(R, b_{H}\right)$ is a $B_{H^{-}}$-subgroup and $\left(P, g_{H}\right) \leq$ $\left(R, b_{H}\right)$.

(ii) Note that in the notation of the proof of Theorem $6.1,\left(R, b_{R}\right)$ is self-centralizing. 
We also observe that there is some redundancy in $(a)$ when $(a *)$ holds:

Remark 7.3 In the notation of Property 7.1 (a) and (a*) suppose $N_{1} \times N_{2} \leq E \leq$ $M_{1} \circ M_{2}$ such that $y$ normalizes $E$ and suppose $\varphi \in \operatorname{Irr}\left(E \mid \theta_{1} \times \theta_{2}\right)$. Then $\varphi^{y}=\varphi$.

Proof: Since $\varphi$ has an extension $\tilde{\varphi}$ to $E Z_{0}$, it follows that we may suppose $Z_{0} \leq E$. Let $\pi_{i}:\left(M_{1} \circ M_{2}\right) \rightarrow M_{i} / Z_{0}$ be the canonical projection and let $E_{i} / Z_{0}=\pi_{i}(E)$ for some $E_{i} \leq M_{i}$. Then

$$
N_{1} \times N_{2} \leq E \leq E_{1} \circ E_{2} \leq M_{1} \circ M_{2}
$$

Let $\zeta$ be the $y$-stable extension of $\theta_{2}$ to $M_{2}$, and set $\tilde{\theta}_{2}=\left.\zeta\right|_{E_{2}}$. Then $\tilde{\theta}_{2}$ is an extension of $\theta_{2}$ to $E_{2}$ which is stabilized by $y$. Since $[y, x]=1$ or $N_{2} Z_{0}$ according as $x \in M_{1}$ or $x \in M_{2}$, it follows that $y$ centralizes $E_{1}$ and $[y, x] \in N_{2} Z_{0}$ for any $x \in E_{2}$. By Lemma $2.7, \varphi^{y}=\varphi$.

Applying Remark 7.3 to the canonical characters of $b$ and $b_{H}$, we see that in the notation of $(a), \theta^{y}=\theta$ and $b_{H}^{y}=b_{H}$ are automatically satisfied if the other parts of $(a *)$ hold.

Before stating the key consequence of Property 7.1, we need the following:

Lemma 7.4 Let $B$ be a block of a finite group $G$ and suppose there are B-subgroups $\left(P, b_{P}\right) \leq\left(R, b_{R}\right)$ such that $R$ is abelian, $b_{P}$ has abelian defect groups and there is $p$ regular element $y \in N_{C_{G}(P)}(R) \backslash C_{G}(R)$ such that $b_{R}^{y}=b_{R}$. Then there is a B-subgroup $\left(Q, b_{Q}\right)$ such that $l\left(b_{Q}\right)>1$.

Proof: For convenience write $L=C_{G}(P)$. Note first that $b_{P}$ is not nilpotent, since $y C_{G}(R) \in N_{L}\left(R, b_{R}\right) / C_{G}(R)$ is not a $p$-element and $\left(R, b_{R}\right)$ is a $b_{P}$-subgroup. Hence by Lemma 2.4 the unique block $\overline{b_{P}}$ of $\bar{L}:=L / P$ dominated by $b_{P}$ is not nilpotent either. But $\overline{b_{P}}$ has abelian defect groups, so by [30, Theorem 3] there is a $\overline{b_{P}}$-subgroup $\left(\bar{Q}, b_{\bar{Q}}\right)$, where $\bar{Q}=Q / P$ for some $Q \geq P$, such that $l\left(b_{\bar{Q}}\right)>1$.

Note that $\overline{C_{L}(Q)} \triangleleft C_{\bar{L}}(\bar{Q})$ and $C_{\bar{L}}(\bar{Q}) / \overline{C_{L}(Q)}$ is a $p$-group. By [33, Lemma 1] there is a $b_{P}$-subgroup $\left(Q, b_{Q}\right)$ such that $b_{\bar{Q}}$ is the unique block of $C_{\bar{L}}(\bar{Q})$ covering the block $b_{\bar{Q}}^{\prime}$ of $\overline{C_{L}(Q)}$ dominated by $b_{Q}$. Note that $l\left(b_{Q}\right)=l\left(b_{\bar{Q}}^{\prime}\right)=l\left(b_{\bar{Q}}\right)>1$. But $P \leq Q$, so $\left(Q, b_{Q}\right)$ is also a $B$-subgroup, and we are done.

We make the key observation, and see also that the conjecture of Puig is a consequence of any of Properties 7.1 (a)-(d).

Corollary 7.5 Suppose one of Property $7.1(a)-(d)$ holds for a block B. If B has non-abelian defect groups, then there is a $B$-subgroup $\left(Q, b_{Q}\right)$ such that $l\left(b_{Q}\right)>1$, and hence $B$ is not nilpotent. In particular, $B$ is nilpotent if and only if $l\left(b_{Q}\right)=1$ for every $B$-subgroup $\left(Q, b_{Q}\right)$.

Proof: If (a) holds, then the result follows immediately from Lemma 7.4, since $B$ is not nilpotent and there is a $B$-subgroup $\left(Q, b_{Q}\right)$ such that $l\left(b_{Q}\right)>1$. If (b) or (c) holds, then $l(B)>1$, so $B$ is not nilpotent, and we may take $\left(Q, b_{Q}\right)=(1, B)$. If $(\mathrm{d})$ 
holds, then this is [30, Theorem 3].

We prove a lemma which will be useful in establishing the given properties. Let $H$ be a finite group, $K \triangleleft H, Z \leq Z(H) \cap K$ and $\bar{K}:=K / Z \leq \bar{H}:=H / Z$. Let $\bar{B} \in \operatorname{Blk}(\bar{K})$ and $B \in \operatorname{Blk}(K)$ dominating $\bar{B}$, and $\left(Q, b_{Q}\right)$ a $B$-subgroup. Let $\gamma: H \rightarrow \bar{H}$ be the natural homomorphism, and write $\bar{X}=\gamma(X)$ for any $X \subseteq H$,

If $Z$ is a $p^{\prime}$-group, then $\left(\bar{Q}, \bar{b}_{Q}\right)$ is defined in Section 2 and it is a $\bar{B}$-subgroup. Suppose $Z$ is a $p$-group. Then $\gamma^{-1}\left(C_{\bar{K}}(\bar{Q})\right) \leq N_{K}(Q Z)$ and $\gamma^{-1}\left(C_{\bar{K}}(\bar{Q})\right) / C_{K}(Q)$ is a $p$-group. Thus $\gamma^{-1}\left(C_{\bar{K}}(\bar{Q})\right)$ has a unique block $\hat{b}_{Q}$ covering $b_{Q}$ and we denote $\bar{b}_{Q}$ the block of $C_{\bar{K}}(\bar{Q})$ corresponding to $\hat{b}_{Q}$, so that by [33, Lemma 1$],\left(\bar{Q}, \bar{b}_{Q}\right)$ is also a $\bar{B}$-subgroup.

In general, since $\bar{K} \cong\left(K / O_{p}(Z)\right) /\left(Z / O_{p}(Z)\right)$ and $Z / O_{p}(Z) \leq Z\left(K / O_{p}(Z)\right)$, it follows that $\left(\bar{Q}, \bar{b}_{Q}\right)$ is defined and is a $\bar{B}$-subgroup.

Lemma 7.6 Let $H$ be a finite group, $K \triangleleft H, Z \leq Z(H) \cap K$. Define $\bar{K}:=K / Z$ and $\bar{H}:=H / Z$. Let $\bar{B} \in \operatorname{Blk}(\bar{K})$ and $B \in \operatorname{Blk}(K)$ dominating $\bar{B}$. Suppose B-subgroups $(P, g) \leq(R, b)$ satisfy Property $7.1(a *)$, and suppose $Z(\bar{K})=Z(K) / Z$. In addition, if $Z=O_{p}(Z)$, then suppose, moreover that $C_{H}(P) / Z=C_{\bar{H}}(\bar{P})$ and $C_{H}(R) / Z=C_{\bar{H}}(\bar{R})$. Then the $\bar{B}$-subgroups $(\bar{P}, \bar{g}) \leq(\bar{R}, \bar{b})$ satisfies Property $7.1(a *)$.

Proof: Let $\bar{B}_{\bar{H}} \in \operatorname{Blk}(\bar{H})$ covering $\bar{B}$, and $B_{H} \in \operatorname{Blk}(H)$ dominating $\bar{B}_{\bar{H}}$ and $\chi \in \operatorname{Irr}\left(\bar{B}_{\bar{H}}\right)$, so that $\chi$ covers some $\psi \in \operatorname{Irr}(\bar{B})$. But $\operatorname{Irr}\left(\bar{B}_{\bar{H}}\right) \subseteq \operatorname{Irr}\left(B_{H}\right)$ and $\operatorname{Irr}(\bar{B}) \subseteq$ $\operatorname{Irr}(B)$, so $B_{H}$ covers $B$.

Let $f$ be the unique block of $Z$ covered by $B$. Then each character $\chi$ in $\operatorname{Irr}(B)$ covers a character in $\operatorname{Irr}(f)$. Since $\operatorname{Irr}(\bar{B}) \subseteq \operatorname{Irr}(B)$, it follows that $f$ is the principal block. Since $(P, g)$ is a $B$-subgroup and $Z \leq Z(K)$ and since $B$ covers $f$, it follows that $g$ covers $f$, and similarly, $b$ covers $f$. The same applies to $B_{H}$ and to $B_{H^{-}}$-subgroups.

Since $C_{K}(P Z)=C_{K}(P)$, it follows that we may suppose $O_{p}(Z) \leq P$. Let $y \in$ $N_{C_{K}(P)}(R)$ such that $y^{4}=1,[y, x] \notin Z(K)$ for some $x \in R, y^{2} \in C_{K}(R)$, and suppose $N_{i}$ and $M_{i}$ are subgroups of $H$, and $\theta_{i} \in \operatorname{Irr}\left(N_{i}\right)$ for $i=1,2$, such that $M_{i} / N_{i}$ is abelian,

$$
Z \leq Z(K) \leq N_{1} \times N_{2} \leq C_{K}(R) \leq C_{H}(R) \leq M_{1} \circ M_{2}
$$

$\theta$ covers $\theta_{1} \times \theta_{2}, Z_{0} \cap N_{2}=1, \theta_{2}$ has a $y$-stable extension $\tilde{\theta}_{2}$ to $M_{2}$ and $[y, x]=1$ or in $Z_{0} N_{2}$ according as $x \in M_{1}$ or $M_{2}$, where $Z_{0} \leq Z\left(M_{1}\right) \cap Z\left(M_{2}\right)$ such that $M_{1} \circ M_{2}$ is the central product over $Z_{0}$ and $\theta$ is the canonical character of $b$.

It suffices to consider the cases $p$-group and a $p^{\prime}$-group separately. Then by [33, Lemma 1 (iii)] and Lemma $2.5,(\bar{P}, \bar{g}) \leq(\bar{R}, \bar{b})$ are $\bar{B}$-subgroups. If $Z$ is a $p^{\prime}$-group, then $C_{K}(R) / Z=C_{\bar{K}}(\bar{R})$. If $Z$ is a $p$-group, then $C_{H}(R) / Z=C_{\bar{H}}(\bar{R}), \gamma^{-1}\left(C_{\bar{H}}(\bar{R})\right)=C_{H}(R)$ and so $\gamma^{-1}\left(C_{\bar{K}}(\bar{R})\right)=C_{K}(R)$. Thus in either case $C_{K}(R) / Z=C_{\bar{K}}(\bar{R})$ and $D(b) Z / Z=$ $D(\bar{b})$. Similarly, $D(\bar{g})=D(g) Z / Z$, and $D(\bar{g})=D(\bar{b})$ is abelian as $D(g)=D(b)$ is abelian.

Let $\bar{y}=\gamma(y)$, so that $\bar{y}^{4}=1, \bar{y} \in C_{\bar{K}}(\bar{P}) \cap N_{\bar{K}}(\bar{R}), \bar{y}^{2} \in C_{\bar{K}}(\bar{R})$. Since $[y, x] \notin Z(K)$ for some $x \in R$ and since $Z(\bar{K})=Z(K) / Z$, it follows that $[\bar{y}, \bar{x}] \notin Z(\bar{K})$ and in particular, $\bar{y} \notin C_{\bar{K}}(\bar{R})$.

Let $\bar{N}_{i}=\gamma\left(N_{i}\right)$ and $\bar{M}_{i}=\gamma\left(M_{i}\right)$. Then $\bar{N}_{i} \triangleleft \bar{M}_{i}$ and $\bar{M}_{i} / \bar{N}_{i}$ is abelian such that

$$
Z(\bar{K}) \leq \bar{N}_{1} \times \bar{N}_{2} \leq C_{\bar{K}}(\bar{R}) \leq C_{\bar{H}}(\bar{R}) \leq \bar{M}_{1} \circ \bar{M}_{2},
$$


where $\bar{M}_{1} \circ \bar{M}_{2}$ is the central product over $\bar{Z}_{0}=\gamma\left(Z_{0}\right)$.

Since $Z \leq Z(K) \leq N_{1} \times N_{2}$ and $\theta$ is the canonical character of $b$, it follows that $\theta$ is the lift of the canonical character $\bar{\theta}$ of $\bar{b}$. Similarly, since $\theta$ covers $\theta_{1} \times \theta_{2}$, it follows that $Z \leq \operatorname{ker}\left(\theta_{1} \times \theta_{2}\right)$ and $\theta_{1} \times \theta_{2}$ is the lift of $\bar{\theta}_{1} \times \bar{\theta}_{2}$ for some $\bar{\theta}_{i} \in \operatorname{Irr}\left(\bar{N}_{i}\right)$. In particular, $\bar{\theta} \in \operatorname{Irr}\left(C_{\bar{K}}(\bar{R}) \mid \bar{\theta}_{1} \times \bar{\theta}_{2}\right)$. If $\varphi \in \operatorname{Irr}\left(M_{1} \circ M_{2} \mid \theta_{1} \times \theta_{2}\right)$, then by Lemma $2.7 \varphi=\psi \circ\left(\tilde{\theta}_{2} \lambda\right)$ for some $\psi \in \operatorname{Irr}\left(M_{1} \mid \theta_{1}\right)$ and $\lambda \in \operatorname{Irr}\left(M_{2} / Z_{0} N_{2}\right)$. Since $[y, x] \in N_{2} Z_{0}$ for all $x \in M_{2}$, it follows that $\lambda^{y}=\lambda$ and so $\tilde{\theta}_{2} \lambda$ is $y$-invariant. $\operatorname{But} Z \leq \operatorname{ker}(\varphi)$, so $Z \cap M_{2} \leq \operatorname{ker}\left(\tilde{\theta}_{2} \lambda\right)$, and $\tilde{\theta}_{2} \lambda$ can be viewed as a character of $\operatorname{Irr}\left(\bar{M}_{2}\right)$, which is a $\bar{y}$-invariant extension of $\bar{\theta}_{2}$ to $\bar{M}_{2}$. Thus Property $7.1(a *)$ holds for $\bar{B}$.

Similarly, if $\left(P, g_{H}\right) \leq\left(R, b_{H}\right)$ are $B_{H^{-}}$blocks such that $D\left(g_{H}\right)=D\left(b_{H}\right)$ is abelian, and $g_{H}$ and $b_{H}$ cover $g$ and $b$, respectively, then there exist $\bar{B}_{\bar{H}}$-subgroups $\left(\bar{P}, \bar{g}_{\bar{H}}\right) \leq$ $\left(\bar{R}, \bar{b}_{\bar{H}}\right)$ such that $\bar{g}_{\bar{H}}$ is dominated by $g_{H}$ and $\bar{b}_{\bar{H}}$ is dominated by $b_{H}$ and $D\left(\bar{g}_{\bar{H}}\right)=$ $D\left(g_{H}\right) Z / Z$ and $D\left(\bar{b}_{\bar{H}}\right)=D\left(b_{H}\right) Z / Z$. Thus $D\left(\bar{g}_{\bar{H}}\right)=D\left(\bar{b}_{\bar{H}}\right)=D\left(b_{H}\right) Z / Z$ is abelian.

Since $g_{H}$ covers $g$, it follows that the canonical character $\theta_{H}$ of $g_{H}$ covers the canonical character $\theta$ of $g$. But $\theta_{H}$ is the lift of the canonical character of $\bar{g}_{\bar{H}}$ and $\theta$ is the lift of the canonical character of $\bar{g}$, so $\bar{g}_{\bar{H}}$ covers $\bar{g}$. Similarly, $\bar{b}_{\bar{H}}$ covers $\bar{b}$. This proves that Property 7.1 (a) holds for $(\bar{P}, \bar{g}) \leq(\bar{R}, \bar{b})$.

\section{Classical groups}

Suppose $p$ is odd. In this section we demonstrate that every nilpotent block of a classical group of has abelian defect groups.

Proposition 8.1 Let $K:=\mathrm{SL}_{n}^{\eta}(q) \leq H \leq G:=\mathrm{GL}_{n}^{\eta}(q)=\mathrm{GL}^{\eta}(V), Z \leq Z(K)$, $B \in \operatorname{Blk}(K), B_{H} \in \operatorname{Blk}(H)$ covering $B$. Let $B_{G} \in \operatorname{Blk}(G)$ be a weakly regular cover of $B_{H}$. Write $R:=A\left(D\left(B_{G}\right)\right) \cap K$. Then Property 7.1 ( $\left.a *\right)$ holds for some B-subgroups $(P, g) \leq(R, b)$ with $C_{H}(P) / Z=C_{\bar{H}}(\bar{P})$ and $C_{H}(R) / Z=C_{\bar{H}}(\bar{R})$, or Property $7.1(b)$ or $(d)$ holds, where $\bar{X}=X Z / Z$ for any $X \leq G$. Moreover, if Property 7.1 (b) holds, then $n=3 d$ with $\operatorname{gcd}(6, d)=1$ and $3 \|(q-\eta)$.

Proof: Suppose $B_{G}$ is labelled by $(s, \kappa)$. Since $B_{H}$ covers $B$, it follows that $D(B)=D\left(B_{H}\right) \cap K$ for some defect group $D\left(B_{H}\right)$. There exists a defect group $D\left(B_{G}\right)$ such that $D\left(B_{H}\right)=D\left(B_{G}\right) \cap H$, so

$$
D(B)=D\left(B_{H}\right) \cap K=D\left(B_{G}\right) \cap K \quad \text { and } \quad D\left(B_{H}\right)=D\left(B_{G}\right) \cap H .
$$

We may suppose $D\left(B_{G}\right) \in \operatorname{Syl}_{p}\left(C_{G}(s)\right)$.

Suppose the decompositions $V=V_{0} \perp V_{+}, D\left(B_{G}\right)=D_{0} \times D_{+}$and $s=s_{0} \times s_{+}$are given as in (6.1). Set $R_{G}=A\left(D\left(B_{G}\right)\right)$. Then $R_{G}$ and $C_{G}\left(R_{G}\right)$ are given by (6.4) with $R$ replaced by $R_{G}$.

In the notation of the proof of Theorem 6.1, suppose each $w_{\Gamma} \leq(p-1)$. Then $D\left(B_{G}\right)$ is abelian, and both $D(B)$ and $D\left(B_{H}\right)$ are abelian. Thus Property $7.1(\mathrm{~d})$ holds. So we suppose that $w_{\Delta} \geq p$ for some $\Delta$. There exists $y \in C_{G}(s) \cap K$ such that $y \in N_{G}\left(R_{G}\right) \backslash C_{G}\left(R_{G}\right),|y|=4,\left.y\right|_{V_{0}}=1_{V_{0}},\left.y\right|_{V_{\Gamma}}=1_{V_{\Gamma}}$ for all $\Gamma \neq \Delta$, and $y$ swaps exactly two factors $K_{\Delta}$ of $C_{G}\left(R_{G}\right)$. Let $R_{H}:=R_{G} \cap H$ and $R=R_{G} \cap K$. 
Let $P_{G}:=P\left(D\left(B_{G}\right)\right)$ be defined by (6.5), so that $P_{G} \leq R_{G}$ and we may suppose $y \in C_{G}\left(P_{G}\right) \cap K$. Let $P:=P_{G} \cap K$ and $P_{H}=P_{G} \cap H$. Since $w_{\Delta} \geq p$, it follows that $\left|\Omega_{1}\left(P_{G}\right)\right| \geq p^{p-1}$, and $P$ is cyclic if and only if $p=3, w_{\Delta}=3, w_{\Gamma}=0$ for all $\Gamma \neq \Delta$ and $P_{G} \not \leq K$.

We claim that $C_{G}(P) \neq C_{G}\left(P_{G}\right)$ if and only if $V_{0}=0, p=3=w_{\Delta}, w_{\Gamma}=0$ when $\Gamma \neq \Delta, \eta=\epsilon, \alpha_{\Delta}=0, e_{\Delta}=1$ and $3 \|(q-\epsilon)$. In particular, $D(B)=3_{+}^{1+2}$ in this case.

Indeed, if $w_{\Delta}>3$, then $P$ is noncyclic and so $C_{G}(P)=C_{G}\left(P_{G}\right)$. Thus $w_{\Delta}=3$, and so $p=3$. If $w_{\Gamma} \neq 0$, then $P$ is also noncyclic and hence $C_{G}(P)=C_{G}\left(P_{G}\right)$. Suppose $p=3=w_{\Delta}$ and $w_{\Gamma}=0$, so that $\left|\Omega_{1}\left(P_{G}\right)\right|=3^{2}$. Define $c \geq 1$ by $3^{c} \|\left(q^{e 3^{\alpha} \Delta}-\epsilon\right)$, and choose $\beta \in \mathbb{F}_{q^{2 e 3}{ }^{\alpha \Delta}}^{\times}$with $|\beta|=3^{c}$. Note that $x_{\beta}:=1_{V_{0}} \times \operatorname{diag}\left\{\beta^{-2}, \beta, \beta\right\} \in P$ and so if $c \geq 2$, then $C_{G}(P)=C_{G}\left(P_{G}\right)$. Thus $3 \|\left(q^{e 3^{\alpha} \Delta}-\epsilon\right)$ and so $\alpha_{\Delta}=0$. Note that $e=1$ or 2 as $p=3$. If $e=2$, then we may suppose $\beta \in \mathrm{SL}_{2}(q)$, since $\mathrm{SL}_{2}(q)$ contains a maximal torus $\mathbb{Z}_{q-\epsilon}$. Thus $\operatorname{det}(\beta)=1, P=P_{G}$ and $C_{G}(P)=C_{G}\left(P_{G}\right)$, a contradiction. So $e=1$ and $3 \|(q-\epsilon)$. But $Z\left(G\left(V_{0}\right)\right) \times Z\left(G\left(V_{+}\right)\right) \leq Z\left(C_{G}(P)\right)$, so $\eta=\epsilon$. Since $3 \mid(q-\eta)$ and $1=D_{0}=O_{3}\left(G\left(V_{0}\right)\right)$, it follows that $V_{0}=0$. Similarly, since $Z(G)=\mathbb{Z}_{q-\eta} \leq Z\left(C_{G}(s)\right)=\mathbb{Z}_{q^{d} \Delta-\epsilon_{\Delta}}$, it follows that $e_{\Delta}=1$ and the claim holds.

Suppose $V_{0}=0, p=3=w_{\Delta}, w_{\Gamma}=0$ when $\Gamma \neq \Delta, \eta=\epsilon, \alpha_{\Delta}=0, e_{\Delta}=1$ and $3 \|(q-\epsilon)$. Then

$$
C_{G}(P)=\mathrm{GL}^{\epsilon}\left(3 d_{\Delta}, q\right)=G, \quad C_{G}\left(P_{G}\right)=\mathrm{GL}^{\epsilon}\left(d_{\Delta}, q\right) \times \mathrm{GL}^{\epsilon}\left(2 d_{\Delta}, q\right),
$$

so $P=O_{3}(Z(G))=O_{3}(Z(K))=\mathbb{Z}_{3}$ and $P_{G}=O_{3}\left(Z\left(C_{G}\left(P_{G}\right)\right)\right)=\mathbb{Z}_{3}^{2}$. In addition, $C_{G}(s) \cong \mathrm{GL}^{\epsilon}\left(3, q^{\delta_{\Delta}}\right)$ and $D\left(B_{G}\right) \in \operatorname{Syl}_{3}\left(\mathrm{GL}^{\epsilon}\left(3, q^{\delta_{\Delta}}\right)\right)$. Since $\alpha_{\Delta}=0$, it follows that $3 \|\left(q^{\delta_{\Delta}}-\epsilon_{\Delta}\right), D\left(B_{G}\right)=\mathbb{Z}_{3} \imath \mathbb{Z}_{3}$ and $|D(B)|=3^{3}$. $\operatorname{But}_{+}^{1+2} \in \operatorname{Syl}_{3}\left(\operatorname{SL}^{\epsilon_{\Delta}}\left(3, q^{\delta_{\Delta}}\right)\right)$, so $D(B) \cong 3_{+}^{1+2}$.

Write $D_{G}=D\left(B_{G}\right), D_{H}=D\left(B_{H}\right)$ and $D=D(B)$. Since $\left[D_{G}: D\right]=3$, it follows that $D_{H}=D$ or $D_{H}=D_{G}$. Note that $D_{G}=\left\langle R_{G}, \sigma\right\rangle$ for some permutation $\sigma$ of order 3. So $D=\langle R, \sigma\rangle, C_{G}\left(D_{G}\right)=C_{G}(D) \cong \mathrm{GL}^{\epsilon}\left(d_{\Delta}, q\right), C_{G}\left(C_{G}(D)\right)=\operatorname{GL}^{\epsilon}(3, q)$ and $\left\langle D_{G}, y\right\rangle \leq C_{G}\left(C_{G}(D)\right)$. Thus $y$ centralizes $C_{G}(D)$ and so $y \in N_{K}\left(D, b_{D}\right)$, where $\left(D, b_{D}\right)$ is a Sylow $B$-subgroup. Since $Z(D) \leq Z(K)$, it follows that $B$ dominates a block $\bar{B} \in \operatorname{Blk}(K / Z(D)), D(\bar{B})=D / Z(D) \cong 3^{2}$ and $\bar{y}$ stabilizes the $\bar{B}$-subgroup $\left(D / Z(D), \bar{b}_{D}\right)$ with $|\bar{y}|=4$, where $\bar{y}=y Z(D)$. In particular, $\bar{B}$ is non-nilpotent. But $D(\bar{B})$ is abelian, so $\ell\left(\bar{b}_{\bar{Q}}\right) \geq 2$ for some $\bar{B}$-subgroup $\left(\bar{Q}, \bar{b}_{\bar{Q}}\right)$. If $\bar{Q} \cong \mathbb{Z}_{3}$, then $C_{K / Z(D)}(\bar{Q})=C_{K}(Q) D / Z(D)=\left(C_{K}(Q) / Z(D)\right) .3$, where $Q=\langle Z(K), w\rangle$ for some $w \in$ $D \backslash Z(D)$ of order 3 . Let $B_{Q}$ be a block of $C_{K}(Q) D$ dominating $\bar{b}_{\bar{Q}}$ and $b_{Q} \in \operatorname{Blk}\left(C_{K}(Q)\right)$ covered by $B_{Q}$. Then $D\left(b_{Q}\right)=Q$ and the canonical character $\theta_{Q}$ of $b_{Q}$ is the only irreducible Brauer character of $b_{Q}$. If $\phi$ is any irreducible Brauer character of $B_{Q}$, then $\phi$ covers $\theta_{Q}$. But $C_{K}(Q)$ contains a representative set of the conjugacy $3^{\prime}$-classes of $C_{K}(Q) D$, so $\ell\left(B_{Q}\right)=1$. In particular, $\ell\left(\bar{b}_{\bar{Q}}\right)=1$. Similarly, if $\bar{Q}=D / Z(D)$, then $\ell\left(\bar{b}_{\bar{Q}}\right)=1$. Thus if $\ell\left(\bar{b}_{\bar{Q}}\right) \geq 2$, then $\bar{Q}=1$ and $\bar{b}_{\bar{Q}}=\bar{B}$. It follows that $\ell(B) \geq \ell(\bar{B}) \geq 2$, and hence Property 7.1 (b) holds.

Since $p$ is odd, it follows that $C_{G}(R)=C_{G}\left(R_{H}\right)=C_{G}\left(R_{G}\right)$. Let $x \in G$ such that for any $u \in R$, there exists $z \in Z(G)$ such that $x^{-1} u x=u z$. Then $x^{-1} u x=c u$ for some $c \in O_{p}\left(\mathbb{F}_{q^{2}}^{\times}\right)$and so $x \in N_{G}(R)=N_{G}\left(R_{G}\right)$. If $\lambda$ is an eigenvalue of $u$ in some algebraic closure of $\mathbb{F}_{q^{2}}$ and $m_{X-\lambda}(u)$ is the multiplicity, then $c \lambda$ is also an eigenvalue of $u$ with the same multiplicity. In particular, $m_{X-1}(u)=m_{X-c}(u)$. It follows that 
if we choose $u \in R$ such that $m_{X-1}(u) \neq m_{X-c}(u)$ for any $c \in \mathbb{F}_{q^{2}}^{\times}$, then $x^{-1} u x=u$. Since $\left|\Omega_{1}(R)\right| \geq p^{p-1}$, it follows that $x \in C_{G}(R)$ and so $C_{G}(R) / Z=C_{G / Z}(R / Z)$ for any $Z \leq Z(G)$, except when $p=3=w_{\Delta}, \alpha_{\Delta}=0$ and $3 \|\left(q^{e}-\epsilon\right)$, in which case $D(B)=3_{+}^{1+2}$. If $3 \nmid(q-\eta)$, then $e=2$ and as shown above $R=R_{G}$, in which case we still have $x \in C_{G}(R)$. If $3 \mid(q-\eta)$, then $3 \|(q-\eta), P \cong \mathbb{Z}_{3}$ and $C_{G}(P) \neq C_{G}\left(P_{G}\right)$, which is discussed above.

Similarly, $C_{G}(P) / Z=C_{G / Z}(P / Z)$ for any $Z \leq Z(G)$ when $C_{G}(P)=C_{G}\left(P_{G}\right)$ (note in this case that for any $u \in P$, we have $m_{X-\lambda}(u) \geq 2$ for some eigenvalue $\left.\lambda\right)$.

Suppose $C_{G}(P)=C_{G}\left(P_{H}\right)=C_{G}\left(P_{G}\right)$ and $C_{G}(R)=C_{G}\left(R_{G}\right)=C_{G}\left(R_{H}\right)$. Thus $C_{G}(R)$ is regular in $G$ and $s \in C_{G}(R)$. Let $(P, g)$ and $(R, b)$ be $B$-subgroups such that $(P, g) \leq(R, b)$.

Let $\left(P, g_{H}\right) \leq\left(R, b_{H}\right)$ be $B_{H}$-subgroups such that $g_{H}$ covers $g$ and $b_{H}$ covers $b$ (see the remark after the definition of Property 7.1), and $\left(P, g_{G}\right) \leq\left(R, b_{G}\right) B_{G^{-}}$-subgroups such that $g_{G}$ covers $g_{H}$ and $b_{G}$ covers $b_{H}$. By [12, Theorem 3.2], we may suppose $b_{G} \subseteq \mathcal{E}_{p}\left(C_{G}(R),(s)\right)$ and so $b_{G}^{y}=b_{G}$ as $y \in C_{G}(s) \cap K$. Now

$$
b_{G}=b(0) \times \prod_{\Gamma} b(\Gamma)^{w_{\Gamma}},
$$

where $b(0)$ is a block of $G_{0}$ labelled by $\left(s_{0}, \kappa\right)$ with defect 0 , and $b(\Gamma)=\mathcal{E}_{p}\left(K_{\Gamma},\left(t_{\Gamma}\right)\right)$ with $t_{\Gamma}$ the restriction of $s$ to $K_{\Gamma}$. Note that if $U_{\Gamma}$ is the underlying space of $K_{\Gamma}$ and view $t_{\Gamma}$ as an element of $G\left(U_{\Gamma}\right)$, then we have $m_{\Gamma}\left(t_{\Gamma}\right)=e_{\Gamma}$. Thus for any generator $x_{\Gamma} \in R_{\Gamma}$

$$
T_{\Gamma}:=C_{K_{\Gamma}}\left(t_{\Gamma}\right)=C_{G\left(U_{\Gamma}\right)}\left(x_{\Gamma} t_{\Gamma}\right) \cong \mathrm{GL}^{\epsilon_{\Gamma}}\left(1, q^{e_{\Gamma} \delta_{\Gamma}}\right)
$$

is a Coxeter torus of both $K_{\Gamma}$ and $G\left(U_{\Gamma}\right)$, and $R_{\Gamma}=O_{p}\left(T_{\Gamma}\right)$ is a defect group of $b(\Gamma)$. In particular, $R_{G}$ is a defect group of $b_{G}$. We may suppose $D(b)=D\left(b_{G}\right) \cap C_{K}(R)$ and $D\left(b_{H}\right)=D\left(b_{G}\right) \cap H$, so that $D(b)=R$ and $D\left(b_{H}\right)=R_{H}$.

Since $\left(P, g_{G}\right) \leq\left(R, b_{G}\right)$ and $D\left(b_{G}\right) \leq C_{G}(P)$, it follows that $D\left(g_{G}\right)=D\left(b_{G}\right)=R_{G}$ and so $D(g)=D\left(b_{G}\right) \cap C_{K}(P)=D\left(b_{G}\right) \cap K=R$ is abelian. Similarly, $D\left(g_{H}\right)=R_{H}=$ $D\left(b_{H}\right)$.

Let $\theta, \theta_{H}$ and $\theta_{G}$ be canonical characters of $b, b_{H}$ and $b_{G}$, respectively. Then $\theta_{G}$ covers $\theta_{H}$ and $\theta_{H}$ covers $\theta$. Now

$$
\theta_{G}=\theta(0) \times \theta(+), \quad \theta(+):=\prod_{\Gamma} \theta(\Gamma)^{w_{\Gamma}}, \quad \theta(\Gamma):= \pm R_{T_{\Gamma}}^{K_{\Gamma}}\left(t_{\Gamma}\right)
$$

where $\operatorname{Irr}(b(0))=\{\theta(0)\}$. If $H_{\Gamma}:=K_{\Gamma} \cap S\left(U_{\Gamma}\right)$, then there exists an element $x_{\Gamma} \in K_{\Gamma}$ permutes all the irreducible constituents of the restriction $\left.\theta(\Gamma)\right|_{H_{\Gamma}}$. Since $w_{\Delta} \geq p$, it follows that $\left.\theta(+)\right|_{C_{S\left(V_{+}\right)}\left(R_{+}\right)}$is irreducible, where $R_{+}=S\left(V_{+}\right) \cap\left(\prod_{\Gamma}\left(R_{\Gamma}\right)^{w_{\Gamma}}\right)$.

Let $K_{0}=S\left(V_{0}\right)$ and $K_{+}=S\left(V_{+}\right)$, so that

$$
C_{K}(R)=\left\langle K_{0} \times C_{K_{+}}\left(R_{+}\right), u_{K}\right\rangle, \quad u_{K}=u_{0} \times u_{+}
$$

with $u_{0} \in G_{0} \backslash K_{0}$ and $u_{+} \in C_{G_{+}}\left(R_{+}\right) \backslash C_{K_{+}}\left(R_{+}\right)$. Note that $G_{0}=\left\langle K_{0}, u_{0}\right\rangle$ and $C_{G_{+}}\left(R_{+}\right)=\left\langle C_{K_{+}}\left(R_{+}\right), u_{+}\right\rangle$.

Let $\theta_{0}$ and $\theta_{+}$be irreducible constituents of $\left.\theta\right|_{K_{0}}$ and $\left.\theta\right|_{C_{K_{+}}\left(R_{+}\right)}$, respectively. Then $\theta \in \operatorname{Irr}\left(C_{K}(R) \mid \theta_{0} \times \theta_{+}\right)$and $\theta_{G}$ covers $\theta_{0} \times \theta_{+}$. But $\left.\theta(+)\right|_{C_{K_{+}}\left(R_{+}\right)}$is irreducible, so 
$\theta_{+}=\left.\theta(+)\right|_{C_{K_{+}}\left(R_{+}\right)}$and $\theta_{+}$has an extension $\theta(+)$ to $C_{G_{+}}\left(R_{+}\right)$. Applying Lemma 2.7 to

$$
K_{0} \times C_{K_{+}}\left(R_{+}\right) \leq C_{K}(R) \leq C_{G}(R)=G_{0} \times C_{G_{+}}\left(R_{+}\right)
$$

(with $Z_{0}=1$ ), we have that $\left.\theta_{G}\right|_{C_{K}(R)}=\theta$. In particular, $\left.\theta_{G}\right|_{C_{K}(R)}$ is irreducible and so is $\left.\theta_{G}\right|_{C_{H}(R)}$. But $\theta_{G}$ covers $\theta_{H}$, so $\left.\theta_{G}\right|_{C_{H}(R)}=\theta_{H}$. Thus $\theta^{y}=\theta, \theta_{H}^{y}=\theta_{H}, b^{y}=b$ and $b_{H}^{y}=b_{H}$. Note that $\left.\theta_{H}\right|_{C_{K}(R)}=\theta$. Thus Property $7.1(a *)$ holds.

Let $V$ be a non-degenerate orthogonal or symplectic space, $G=I_{0}(V)$ and let $G^{*}$ be the dual group of $G$. Then

$$
\mathrm{Sp}_{2 n}(q)^{*}=\mathrm{SO}_{2 n+1}(q), \quad \mathrm{SO}_{2 n+1}(q)^{*}=\mathrm{Sp}_{2 n}(q), \quad \mathrm{SO}_{2 n}^{\eta}(q)^{*}=\mathrm{SO}_{2 n}^{\eta}(q) .
$$

If $B$ is a block of $I_{0}(V)$, then there exists a semisimple $p^{\prime}$-element $s \in I_{0}(V)^{*}$ such that

$$
B \subseteq \mathcal{E}_{p}\left(I_{0}(V),(s)\right)
$$

Let $\left(D, b_{D}\right)$ be a Sylow $B$-subgroup of $I_{0}(V)$. Then $V$ and $D$ have corresponding decompositions

$$
V=V_{0} \perp V_{+}, \quad D=D_{0} \times D_{+} .
$$

We have $V_{0}=C_{V}(D), V_{+}=[V, D], D_{0}=\left\{1_{V_{0}}\right\}$ and $D_{+} \leq I_{0}\left(V_{+}\right)$. Let $G_{0}:=I_{0}\left(V_{0}\right)$, $G_{+}:=I_{0}\left(V_{+}\right), C_{+}:=C_{I_{0}\left(V_{+}\right)}\left(D_{+}\right)$and let $V^{*}$ be the underlying space of $I_{0}(V)^{*}$.

Let $z \in D$ be a primitive element. Then $z \in Z(D)$ with $|z|=p$ (cf. [17, p.176]). Thus

$$
z=z_{0} \times z_{+}, \quad L:=C_{G}(z)=L_{0} \times L_{+}, \quad L_{0}=G_{0}, \quad L_{+}:=\mathrm{GL}^{\epsilon}\left(m, q^{e}\right),
$$

where $z_{0}=1_{V_{0}}, z_{+} \leq D_{+}$and $\operatorname{dim} V_{+}=2 \mathrm{em}$. Then $L$ is a regular subgroup of $G$ and we may suppose $s \in L^{*} \leq G^{*}$. In particular,

$$
V^{*}=U_{0} \perp U_{+} \quad \text { and } \quad s=s_{0} \times s_{+},
$$

where $U_{0}=V_{0}^{*}, s_{0} \in L_{0}^{*}=I_{0}\left(U_{0}\right), U_{+}$is the underlying space of $L_{+}^{*}$ and $s_{+} \in L_{+}^{*} \leq$ $I_{0}\left(U_{+}\right)$.

Let $C_{I\left(U_{+}\right)}\left(s_{+}\right)=\prod_{\Gamma} C_{\Gamma}$ and let $U_{\Gamma}$ be the underlying vector space of $C_{\Gamma}$, so that

$$
C_{\Gamma}=\mathrm{GL}^{\epsilon_{\Gamma}}\left(m_{\Gamma}\left(s_{+}\right), q^{\delta_{\Gamma}}\right) \quad \text { or } \quad I\left(U_{\Gamma}\right)
$$

according as $\Gamma \neq X \pm 1$ or $\Gamma=X \pm 1$.

Proposition 8.2 Let $K:=\Omega_{2 n}^{\eta}(q):=\Omega^{\eta}(V) \leq H \leq J_{0}(V), B_{K} \in \operatorname{Blk}(K)$ and $B_{H} \in \operatorname{Blk}(H)$ covering $B_{K}$. Write $R:=A\left(D\left(B_{K}\right)\right)$. Then either Property 7.1 ( $\left.a *\right)$ holds for some $B_{K}$-subgroups $(P, g) \leq(R, b)$ or Property $7.1(d)$ holds, where $P$ is some subgroup of $R$.

Proof: Let $G:=\mathrm{SO}_{2 n}^{\eta}(q):=\mathrm{SO}(V)$ and $B \in \operatorname{Blk}(G)$ covering $B_{K}$. Then $D\left(B_{K}\right)=$ $D \cap K$ for some defect group $D:=D(B)$. Since $G$ is self dual, we have $V=V^{*}, U_{0}=$ $V_{0}, U_{+}=V_{+}$in $(8.3)$. 
(1). Since $|G: K|=2$, it follows that $D=D\left(B_{K}\right)$ and $D=D\left(B_{H}\right) \cap G$ for some $D\left(B_{H}\right)$. In the notation above we have

$$
C_{K}(z) \leq L=L_{0} \times L_{+} .
$$

Let $K_{0}=\Omega\left(V_{0}\right), K_{+}=\Omega\left(V_{+}\right)$and $M_{+}:=\mathrm{SL}^{\epsilon}\left(m, q^{e}\right) \leq L_{+} \cap K_{+}$, so that

$$
M_{+} \leq C_{K_{+}}\left(z_{+}\right) \leq L_{+}, \quad C_{K}(z)=\left\langle K_{0} \times C_{K_{+}}\left(z_{+}\right), t_{0} \times t_{+}\right\rangle
$$

and $\left[L_{+}: C_{K_{+}}\left(z_{+}\right)\right] \leq 2$, where $t_{0} \in L_{0} \backslash K_{0}$ and $t_{+} \in L_{+}$. Let $\left(z, B_{z}\right)$ be a major subsection of $B_{K}$. Then $B_{z}$ covers a block $B_{0} \times B_{+}$of $K_{0} \times C_{K_{+}}\left(z_{+}\right)$with $B_{0} \in$ $\operatorname{Blk}\left(K_{0}\right)$ and $B_{+} \in \operatorname{Blk}\left(C_{K_{+}}\left(z_{+}\right)\right)$such that $D\left(B_{z}\right)=D\left(B_{0} \times B_{+}\right)=D$. Note since $\left[L_{+}: C_{K_{+}}\left(z_{+}\right)\right] \leq 2$, it follows that $D\left(B_{+}\right)=D_{+} \not 3_{+}^{1+2}$.

By [15, Lemma 4.1], there exists a $B$-subgroup $\left(z, B_{L}\right)$ such that $B_{L}$ covers $B_{z}$. Thus $\left(z, B_{L}\right)$ is a major subsection of $B$.

Let $R:=A(D)$, so that $z \in R$ and

$$
R=D_{0} \times \prod_{\Gamma}\left(R_{\Gamma}\right)^{w_{\Gamma}}, \quad C_{G}(R)=\mathrm{SO}\left(V_{0}\right) \times \prod_{\Gamma}\left(K_{\Gamma}\right)^{w_{\Gamma}}
$$

where $R_{\Gamma}=O_{p}\left(K_{\Gamma}\right), m_{\Gamma}\left(s_{+}\right)=w_{\Gamma} e_{\Gamma}$ or $2 w_{\Gamma} e_{\Gamma}$ according as $\Gamma \neq X \pm 1$ or $\Gamma=X \pm 1$, $K_{\Gamma} \cong \mathrm{GL}^{\epsilon}\left(\delta_{\Gamma}^{\prime}, q^{e p^{\alpha} \Gamma}\right)$. Thus $R=O_{p}\left(C_{G}(R)\right), C_{G}(R)$ is a regular subgroup of $G$ and we may suppose $s \in C_{G}(R)$. Set $R_{+}=\prod_{\Gamma}\left(R_{\Gamma}\right)^{w_{\Gamma}}$, so that $R_{+} \leq C_{K_{+}}\left(z_{+}\right)$and

$$
K_{0} \times C_{K_{+}}\left(R_{+}\right) \leq C_{K}(R) \leq C_{G}(R)=L_{0} \times C_{L_{+}}\left(R_{+}\right) .
$$

Let $\left(R_{+}, b_{+}\right)$be a $B_{+}$-subgroup, so that

$$
\left(B_{0} \times b_{+}\right)^{K_{0} \times C_{K_{+}}\left(z_{+}\right)}=B_{0} \times B_{+}
$$

and $b_{+} \in \operatorname{Blk}\left(C_{K_{+}}\left(R_{+}\right)\right)$as $C_{K_{+}}\left(R_{+}\right) \leq C_{K_{+}}\left(z_{+}\right)$. Now $K_{0} \times C_{K_{+}}\left(z_{+}\right)$is normal in $C_{K}(z), R \leq K_{0} \times C_{K_{+}}\left(z_{+}\right)$and $B_{z}$ covers $B_{0} \times B_{+}$. It follows by [15, Lemma 4.1] that there exists a $B_{z}$-subgroup $(R, b)$ such that $b$ covers $B_{0} \times b_{+}$, so that $(R, b)$ is a $B_{K}$-subgroup.

Similarly, there exists a $B_{L}$-subgroup $\left(R, b_{G}\right)$ such that $b_{G}$ covers $b$. Thus $b_{G} \subseteq$ $\mathcal{E}_{p}\left(C_{G}(R),(s)\right), R=D\left(b_{G}\right)$, and so $R=D(b)=D\left(B_{0} \times b_{+}\right)$.

Since $L=L_{0} \times L_{+}$with $L_{0}=G_{0}=\mathrm{SO}\left(V_{0}\right)$, it follows that $B_{L}=B_{L_{0}} \times B_{L_{+}}$with $B_{L_{0}} \in \operatorname{Blk}\left(G_{0}\right)$ and $B_{L_{+}} \in \operatorname{Blk}\left(L_{+}\right)$. But $B_{L}$ covers $B_{z}$ and $B_{z}$ covers $B_{0} \times B_{+}$, so $B_{L_{0}}$ covers $B_{0}$ and $B_{L_{+}}$covers $B_{+}$. In particular, $D_{+}=D\left(B_{+}\right)=D\left(B_{L_{+}}\right)$. Similarly, $b_{G}=b_{G_{0}} \times b_{G_{+}}$with $b_{G_{0}}=B_{L_{0}} \in \operatorname{Blk}\left(G_{0}\right)$ and $b_{G_{+}} \in \operatorname{Blk}\left(C_{L_{+}}\left(R_{+}\right)\right)$, and $b_{G_{+}}$covers $b_{+}$.

Suppose $D$ is non-abelian, so that $w_{\Delta} \geq p$ for some $\Delta$. Let $P:=D_{0} \times P_{+} \leq R$ and $(P, g) \leq(R, b)$, where $P_{+}:=P\left(D_{+}\right)$is given by (6.5). By Proposition 8.1, there exists $B_{+}$-subgroup $\left(P_{+}, g_{+}\right)$such that $\left(P_{+}, g_{+}\right) \leq\left(R_{+}, b_{+}\right)$and $D\left(g_{+}\right)=D\left(b_{+}\right)=R_{+}$. By the remark after Property 7.1 , we may choose $(P, g)$ such that $g$ covering $B_{0} \times g_{+}$, so $D(g)=D_{0} \times D\left(g_{+}\right)=D_{0} \times D\left(b_{+}\right)=D(b)=R$.

In addition, there exists $y \in C_{L_{+}}\left(s_{+}\right) \cap M_{+}$such that $y \in N_{C_{L_{+}}\left(P_{+}\right)}\left(R_{+}\right) \backslash C_{L_{+}}\left(R_{+}\right)$, $|y|=4, y^{2} \in C_{L_{+}}\left(R_{+}\right),\left.y\right|_{V_{0}}=1_{V_{0}},\left.y\right|_{V_{\Gamma}}=1_{V_{\Gamma}}$ for all $\Gamma \neq \Delta$ and $y$ swaps exactly two factors $K_{\Delta}$ of $C_{G}(R)$. Since $y \in C_{L_{+}}\left(s_{+}\right)$, it follows that $\left(b_{G_{+}}\right)^{y}=b_{G_{+}}$. 
Let $\theta_{G}$ and $\theta$ be canonical characters of $b_{G}$ and $b$, respectively. Then $\theta_{G} \operatorname{covers} \theta$,

$$
\theta_{G}=\theta_{G_{0}} \times \theta_{G_{+}}
$$

with $\operatorname{Irr}\left(b_{G_{0}}\right)=\left\{\theta_{G_{0}}\right\}$ and $\theta_{G_{+}}$the canonical character of $b_{G_{+}}$. If $\operatorname{Irr}\left(B_{0}\right)=\left\{\theta_{0}\right\}$ and $\theta_{+}$ is the canonical character of $b_{+}$, then $\theta_{G_{+}}$covers $\theta_{+}$. Since $C_{K_{+}}\left(R_{+}\right)=C_{C_{K_{+}}\left(z_{+}\right)}\left(R_{+}\right)$, it follows by the proof of Proposition 8.1 that $\theta_{+}=\left.\theta_{G_{+}}\right|_{C_{K_{+}}\left(R_{+}\right)}$and $\theta_{+}^{y}=\theta_{+}$.

Now

$$
C_{K}(R)=\left\langle K_{0} \times C_{K_{+}}\left(R_{+}\right), u_{K}\right\rangle, \quad u_{K}=u_{0} \times u_{+}
$$

with $\left[C_{K}(R): K_{0} \times C_{K_{+}}\left(R_{+}\right)\right] \leq 2$, where $u_{0} \in G_{0}$ and $u_{+} \in C_{L_{+}}\left(R_{+}\right)$. If $C_{K}(R)=$ $K_{0} \times C_{K_{+}}\left(R_{+}\right)$, then $\theta=\theta_{0} \times \theta_{+}$and $\theta^{y}=\theta$. If $\left[C_{K}(R): K_{0} \times C_{K_{+}}\left(R_{+}\right)\right]=2$, then $L_{0}=\left\langle K_{0}, u_{0}\right\rangle$ and $C_{L_{+}}\left(R_{+}\right)=\left\langle C_{K_{+}}\left(R_{+}\right), u_{+}\right\rangle$. Now $\theta \in \operatorname{Irr}\left(C_{K}(R) \mid \theta_{0} \times \theta_{+}\right)$and $\theta_{G} \in \operatorname{Irr}\left(C_{G}(R) \mid \theta\right)$ and $\theta_{+}$has an extension $\theta_{G_{+}}$to $C_{L_{+}}\left(R_{+}\right)$, so by Lemma 2.7, $\left.\theta_{G}\right|_{C_{K}(R)}=\theta$ and hence $\theta^{y}=\theta$ and $y \in N_{K}(R, b)$.

(2). Suppose $K \leq H \leq J_{0}(V)$. Let $\left(P, g_{H}\right) \leq\left(R, b_{H}\right)$ be $B_{H}$-subgroups such that $g_{H}$ covers $g$ and $b_{H}$ covering $b$. Since $J_{0}(V) / Z\left(\bar{J}_{0}(V)\right) K$ is a 2-group and $p$ is odd, it follows that $D\left(g_{H}\right)=O_{p}(Z(H)) D(g)=O_{p}(Z(H)) D(b)=D\left(b_{H}\right)$ and both are abelian, and the canonical character $\theta_{H}$ of $b_{H}$ covers $\theta$. Now

$$
K_{0} \times C_{K_{+}}\left(R_{+}\right) \leq C_{K}(R) \leq C_{H}(R) \leq C_{J_{0}(V)}(R) \leq J_{0}\left(V_{0}\right) \times C_{J_{0}\left(V_{+}\right)}\left(R_{+}\right) .
$$

By $[17,(1 \mathrm{~A})], C_{J_{0}\left(V_{+}\right)}\left(z_{+}\right)=\left\langle L_{+}, \tau_{+}\right\rangle$with $\left[\tau_{+}, L_{+}\right]=1$ and so $C_{J_{0}\left(V_{+}\right)}\left(R_{+}\right)$is a central product $C_{L_{+}}\left(R_{+}\right) \circ\left\langle\tau_{+}\right\rangle$. In particular, $\theta_{G_{+}}$has an extension $\tilde{\theta}_{G_{+}}$to $C_{J_{0}\left(V_{+}\right)}\left(R_{+}\right)$, and $\tilde{\theta}_{G_{+}}$is also an extension of $\theta_{+}$. Moreover, $y$ stabilizes $\tilde{\theta}_{G_{+}}$, since $\tilde{\theta}_{G_{+}}$is a central produce $\theta_{G_{+}} \circ \beta$ for some $\beta \in \operatorname{Irr}\left(\left\langle\tau_{+}\right\rangle\right)$and $\theta_{G_{+}}^{y}=\theta_{G_{+}}$. Since $y \in M_{+} \leq K_{+}$and $y$ normalizes $K_{+}$, it follows that $[y, x] \in C_{K_{+}}\left(R_{+}\right)=C_{L_{+}}\left(R_{+}\right) \cap K_{+}$for any $x \in C_{L_{+}}\left(R_{+}\right)$. But $C_{J_{0}\left(V_{+}\right)}\left(R_{+}\right)=C_{L_{+}}\left(R_{+}\right) \circ\left\langle\tau_{+}\right\rangle$, so $[y, x] \in C_{K_{+}}\left(R_{+}\right)$for all $x \in C_{J_{0}\left(V_{+}\right)}\left(R_{+}\right)$. It follows by Remark 7.3 that $y$ stabilizes $\theta_{H}$ and so $b_{H}^{y}=b_{H}$. Thus Property 7.1 (a*) holds.

(3). Suppose $w_{\Gamma}<p$ for any $\Gamma$ with $m_{\Gamma}\left(s_{+}\right) \neq 0$. Then $D=D\left(B_{K}\right)$ is abelian, and so $D\left(B_{H}\right)=D O_{p}(Z(H))$ is abelian. Thus Property $7.1(\mathrm{~d})$ holds.

Proposition 8.3 Let $K:=\Omega_{2 n+1}(q)=\Omega(V)$ or $K:=\operatorname{Sp}_{2 n}(q)=\operatorname{Sp}(V)$, and

$$
K \leq H \leq J_{0}(V),
$$

$B_{K} \in \operatorname{Blk}(K)$ and $B_{H} \in \operatorname{Blk}(H)$ covering $B_{K}$, where $H=\mathrm{SO}(V)$ when $K=\Omega(V)$. Write $R:=A\left(D\left(B_{K}\right)\right)$. Then either Property 7.1 ( $\left.a *\right)$ holds for some $B_{K}$-subgroups $(P, g) \leq(R, b)$ or Property $7.1(d)$ holds, where $P$ is some subgroup of $R$.

Proof: Suppose $V$ is orthogonal. Replacing $G$ by $H$ in the proof (1) of Proposition 8.2 with some obvious modifications, we have that Property $7.1(a *)$ holds for $(P, g) \leq$ $(R, b)$. Suppose $V$ is symplectic, so that $H / K$ is cyclic. Applying the proofs $(1)$ and (2) of Proposition 8.2 with some obvious modifications, we have that Property $7.1(a *)$ holds for $(P, g) \leq(R, b)$. 
If $D\left(B_{K}\right)$ is abelian, then $D\left(B_{K}\right)=D\left(B_{H}\right) \cap K$ for some $D\left(B_{H}\right)$. Since the outerdiagonal group of $K$ is order 2 , it follows that $D\left(B_{H}\right) \leq K Z(H)$ and so $D\left(B_{H}\right)=$ $D\left(B_{K}\right) O_{p}(Z(H))$ is abelian.

Theorem 8.4 Let $G=I_{0}(V), B \in \operatorname{Blk}(G)$, and $\left(D, b_{D}\right)$ a Sylow B-subgroup. Follow the notation in (8.1), (8.2) and (8.3). Then the following are equivalent:

(a) $B$ is nilpotent.

(b) $C_{I_{0}(V)}(D)=G_{0} \times C_{I_{0}\left(V_{+}\right)}\left(D_{+}\right)$is a regular subgroup of $I_{0}(V)$ and $s \in C_{I_{0}(V)}(D)^{*} \leq$ $L^{*}$ satisfies the following conditions.

(i) Suppose $I_{0}(V)=\mathrm{Sp}_{2 n}(q)$ or $\mathrm{SO}_{2 n+1}(q)$. Then

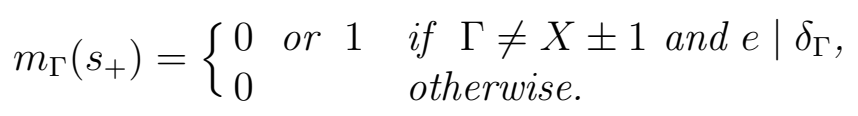

(ii) Suppose $I_{0}(V)=\mathrm{SO}_{2 n}^{\eta}(q)$. Then

$$
\begin{cases}m_{\Gamma}\left(s_{+}\right)=0 \text { or } 1 & \text { if } \Gamma \neq X \pm 1 \text { and } e \mid \delta_{\Gamma} \\ m_{X-1}\left(s_{+}\right)+m_{X+1}\left(s_{+}\right)=0 \text { or } 2 & \text { if } p \mid(q-\epsilon) \\ m_{\Gamma}\left(s_{+}\right)=0 & \text { otherwise, }\end{cases}
$$

where $\epsilon$ is the type of the underlying space of $\left(s_{+}\right)_{X \pm 1}$ when $m_{X-1}\left(s_{+}\right)+$ $m_{X+1}\left(s_{+}\right)=2$.

(c) $C_{I_{0}(V)}(D)=G_{0} \times C_{I_{0}\left(V_{+}\right)}\left(D_{+}\right)$is a regular subgroup of $I_{0}(V)$ and $s \in C_{I_{0}(V)}(D)^{*} \leq$ $L^{*}$ such that $T_{+}^{*}:=C_{I_{0}\left(U_{+}\right)}\left(s_{+}\right)$is a maximal torus of $I_{0}\left(U_{+}\right)$. In particular, if $\theta=\theta_{0} \times \theta_{+}$is the canonical character of $b_{D}$ with $\theta_{0} \in \operatorname{Irr}\left(G_{0}\right)$ and $\theta_{+} \in \operatorname{Irr}\left(C_{+}\right)$, then $\theta_{0}$ has defect zero and $\theta_{+}= \pm R_{T_{+}}^{C_{+}}\left(s_{+}\right)$, where $C_{+}=C_{I_{0}\left(V_{+}\right)}\left(D_{+}\right)$and $T_{+} \leq$ $C_{+}$is a dual of $T_{+}^{*}$.

Proof: Suppose $B$ is nilpotent. By Propositions 8.2 and $8.3, D$ is abelian, so $m_{\Gamma}\left(s_{+}\right)=e_{\Gamma}$ or 0 when $\Gamma \neq X \pm 1$ and $m_{\Gamma}\left(s_{+}\right)=2 e$ or 0 when $\Gamma=X \pm 1$.

Suppose $m_{\Gamma}\left(s_{+}\right)=e_{\Gamma} \geq 2$ with $\Gamma \neq X \pm 1$. As shown in the proof of Theorem 6.1 there exists a $p^{\prime}$-element $\tau_{\Gamma} \in C_{\Gamma}^{*}$ of order $e_{\Gamma}$ normalizing $D_{\Gamma}:=D \cap C_{\Gamma}^{*}$, so that there exists a $p^{\prime}$-element of order $e_{\Gamma}$ normalizing the Sylow $B$-subgroup $\left(D, b_{D}\right)$, a contradiction, so $e_{\Gamma} \leq 1$ and $e \mid \delta_{\Gamma}$.

Suppose $\Gamma=X \pm 1$, so that $m_{\Gamma}\left(s_{+}\right)=2 e$ or 0 and $C_{\Gamma}=I\left(U_{\Gamma}\right)$.

Suppose, moreover that $G=\mathrm{SO}_{2 n}^{\eta}(q)$, so that $G=G^{*}$. Let $\Delta=X \pm 1$ and suppose $m_{\Delta}\left(s_{+}\right)=2 e$. By $[17,(1.14)]$,

$$
\left|N_{I\left(U_{\Delta}\right)}\left(\left\langle z_{\Delta}\right\rangle\right) / C_{I\left(U_{\Delta}\right)}\left(z_{\Delta}\right)\right|=2 e .
$$

If $e \geq 2$, then there exists $y_{\Delta} \in N_{I_{0}\left(U_{\Delta}\right)}\left(\left\langle z_{\Delta}\right\rangle\right) \backslash C_{I_{0}\left(U_{\Delta}\right)}\left(z_{\Delta}\right)$ of order $e$, so that $y_{\Delta}$ normalizes the Sylow subgroup $D_{\Delta}$ of $C_{I_{0}\left(U_{\Delta}\right)}\left(z_{\Delta}\right)$. Let $y_{\Gamma}=1 \in I_{0}\left(U_{\Gamma}\right)$ and $y=$ $1_{V_{0}} \times \prod_{\Gamma} y_{\Gamma}$. Then

$$
y \in\left(N_{G}(D) \cap C_{G}(s)\right) \backslash C_{G}(D)
$$


and $y$ normalizes $\left(D, b_{D}\right)$. Since $|y|=e \neq 1$, it follows that $B$ is not nilpotent, which is impossible. Thus $e=1, I_{0}\left(U_{\Delta}\right)=\mathrm{SO}^{\epsilon}(2, q)$ with $\epsilon=\eta\left(U_{\Delta}\right)$, so $p \mid(q-\epsilon)$.

Similarly, suppose $m_{X-1}\left(s_{+}\right)=m_{X+1}\left(s_{+}\right)=2$. Since $\Omega_{4}^{-}(q)=P \mathrm{SL}_{2}\left(q^{2}\right), \Omega_{4}^{+}(q)=$ $\mathrm{SL}_{2}(q) \circ \mathrm{SL}_{2}(q)$ and $\mathrm{SO}_{4}^{ \pm}(q)=\Omega_{4}^{ \pm}(q) .2$, it follows that there exists an element $w \in$ $\left(N_{G}(D) \cap C_{G}(s)\right) \backslash C_{G}(D)$ of order 2 such that $w \in N_{G}\left(D, b_{D}\right)$, a contradiction.

If $G=\operatorname{Sp}_{2 n}(q)$ or $\mathrm{SO}_{2 n+1}(q)$, then by [17, (1.14)] again, there exists an element $w \in N_{G}(D) \backslash C_{G}(D)$ of order $2 e$ such that $w$ normalizes $\left(D, b_{D}\right)$, which is impossible. Thus $m_{X \pm 1}\left(s_{+}\right)=0$ and (b) holds.

Suppose $(b)$ holds. Then $T_{+}^{*}:=C_{C_{+}^{*}}\left(s_{+}\right)$is a maximal torus of $I_{0}\left(U_{+}\right)$and so $D$ is abelian. Since $C_{G}(D)$ is regular in $G$, we may suppose $s \in C_{G}(D)^{*}$ and so

$$
b_{D} \subseteq \mathcal{E}_{p}\left(C_{G}(D),(s)\right) .
$$

Thus $\theta_{+}= \pm R_{T_{+}}^{C_{+}}\left(s_{+}\right)$and $\theta=\theta_{0} \times \theta_{+}$is the canonical character of $b_{D}$, where $\theta_{0} \in \operatorname{Irr}\left(G_{0}\right)$ has defect 0 . In particular, $N_{G}(D, \theta)=C_{G}(D)$ and $B$ is nilpotent.

Proposition 8.5 Let $K:=\operatorname{Spin}^{\eta}(V) \triangleleft H$ such that $H / K$ is abelian, $C_{H}(K) \leq Z(H)$ and $H / Z(H) \leq \mathrm{SO}(V)$ or $J_{0}(V) / Z\left(J_{0}(V)\right)$ according as $\operatorname{dim} V$ is odd or even. Let $B \in \operatorname{Blk}(K), B_{H} \in \operatorname{Blk}(H)$ covering $B$, and $Z \leq Z(K)$ such that $K_{c}:=K / Z=\Omega^{\eta}(V)$, so that $|Z|=\operatorname{gcd}(2, q-\eta)$. Write $R:=A(D(B))$. Then either Property 7.1 (a*) holds for some $B_{K}$-subgroups $(P, g) \leq(R, b)$ or Property $7.1(d)$ holds, where $P$ is some subgroup of $R$.

Proof: Let $D:=D(B), G:=\mathrm{SO}^{\eta}(V)$ and $Z_{+} \leq Z\left(D_{0}(V)\right)$ such that $G=$ $D_{0}(V) / Z_{+}$, so that $Z=Z_{+} \cap K$ and $Z_{+} \cong \mathbb{Z}_{q-1}$.

We may suppose $D=D Z / Z \leq K_{c}$. Thus $D$ is of defect type in $K_{c}$, where a $p$-subgroup $Q$ of $K_{c}$ is of defect type if $Q$ is a Sylow $p$-subgroup of a centralizer $C_{K_{c}}(t)$ of a semisimple $p^{\prime}$-element $t$. So $D$ is of defect type of $G$, and $D$ has a primary element $z \in Z(D)$ (see [17, Section 5]). Thus we have the corresponding decompositions $V=V_{0} \perp V_{+}, D=D_{0} \times D_{+}, z=z_{0} \times z_{+}, C_{G}(z)=L_{0} \times L_{+}$given by (8.1) and (8.2). Since $K$ is universal, it follows by [20, Theorem 4.2.2] and [17, (2E)] that

$$
C:=C_{K}(z)=L_{C} T_{C}, \quad L_{C}=L_{1} \times \mathrm{SL}^{\epsilon}\left(m, q^{e}\right), \quad L_{1}=\operatorname{Spin}\left(V_{0}\right),
$$

where $T_{C}$ is an abelian $r^{\prime}$-group inducing inner-diagonal automorphisms on $\operatorname{SL}^{\epsilon}\left(m, q^{e}\right)$ and $L_{1}$. Here for simplicity, we identify $z$ with its preimage (with the same order) in $K$. Since $p$ is odd and $D \leq C$, it follows that $D_{+} \cap L_{1}=1$ and so $L_{1} L_{2}=L_{1} \times L_{2}$, where $L_{2}=\operatorname{SL}^{\epsilon}\left(m, q^{e}\right) D_{+} \leq L_{+}$. Let $L=L_{1} \times L_{2} \leq C$, so that $C=L T_{C}$. Let $\left(z, B_{z}\right)$ be a major subsection of $B$, and $B_{L}=B_{1} \times B_{2}$ a block of $L$ covered by $B_{z}$, where $B_{1} \in \operatorname{Blk}\left(L_{1}\right)$ and $B_{2} \in \operatorname{Blk}\left(L_{2}\right)$. We may suppose $D\left(B_{2}\right)=D_{+} \cap L_{2}$, so that $D\left(B_{2}\right)=D_{+}$.

Suppose $B_{2}$ satisfies Property $7.1(a *)$. Let $R_{2}:=A\left(D_{+}\right) \leq D\left(B_{2}\right), R:=R_{1} \times R_{2}$, $P_{2}:=P\left(D_{+}\right) \leq R_{2}, P=P_{1} \times P_{2} \leq R$ and let $\left(P, g_{L}\right) \leq\left(R, b_{L}\right)$ be $B_{L}$-subgroups, where $P_{1}=R_{1}=D_{0}$. So $g_{L}=g_{1} \times g_{2}, b_{L}=b_{1} \times b_{2}$ with $g_{1}=b_{1}=B_{1}, g_{2}^{L_{2}}=b_{2}^{L_{2}}=B_{2}$ and $D\left(b_{2}\right)=R_{2}=D\left(g_{2}\right)$. In addition, there exists $y \in\left(N_{L_{2}}\left(R_{2}\right) \cap C_{L_{2}}\left(P_{2}\right)\right) \backslash C_{L_{2}}\left(R_{2}\right)$ such that $y^{4}=1, y^{2} \in C_{L_{2}}\left(R_{2}\right)$, and $b_{2}^{y}=b_{2}$. Thus $b_{L}^{y}=b_{L}$ and $D\left(b_{L}\right)=R=D\left(g_{L}\right)$. 
(1). For $t \in T_{C}$ write $t=t_{1} t_{2}$ such that $\left[t_{1}, t_{2}\right]=1$ and $t_{2}$ induces inner-diagonal automorphism on $L_{2}$. Let $J_{i}=\left\langle L_{i}, t_{i}: t=t_{1} t_{2} \in T_{C}\right\rangle$, so that $C \triangleleft J:=J_{1} \times J_{2}$ and $L_{2} \leq J_{2} \leq L_{+}$. Let $B_{J} \in \operatorname{Blk}(J)$ be a weakly regular cover of $B_{z},(P, g) \leq(R, b) B_{z^{-}}$ subgroups such that $g$ covers $g_{L}$ and $b$ covers $b_{L}$, and $\left(P, g_{J}\right) \leq\left(R, b_{J}\right) B_{J}$-subgroups such that $g_{J}$ covers $g$ and $b_{J}$ covers $b$, so that $g_{J}$ covers $g_{L}$ and $b_{J}$ covers $b_{L}$.

If $g_{J}=g_{J_{1}} \times g_{J_{2}}$ and $b_{J}=b_{J_{1}} \times b_{J_{2}}$ for some $g_{J_{i}} \in \operatorname{Blk}\left(C_{J_{i}}\left(P_{i}\right)\right)$ and $b_{J_{i}} \in$ $\operatorname{Blk}\left(C_{J_{i}}\left(R_{i}\right)\right)$, then $g_{J_{2}}$ covers $g_{2}$ and $b_{J_{2}}$ covers $b_{2}$ and by Proposition 8.1 and its proof (1), $D\left(g_{J_{2}}\right)=D\left(b_{J_{2}}\right)$ is abelian $\left.\theta_{J_{2}}\right|_{C_{L_{2}}\left(R_{2}\right)}=\theta_{2}$ and $\theta_{J_{2}}^{y}=\theta_{J_{2}}$, where $\theta_{J_{i}}$ and $\theta_{i}$ are canonical characters of $b_{J_{i}}$ and $b_{i}$, respectively. Thus $D\left(g_{J}\right)=D\left(b_{J}\right)$ is abelian. But $P \leq R \leq D\left(g_{J}\right)$ and $D\left(g_{J}\right)$ is abelian, so

$$
D(g)=D\left(g_{J}\right) \cap C_{K}(P)=D\left(g_{J}\right) \cap K=D\left(b_{J}\right) \cap K=D\left(b_{J}\right) \cap C_{K}(R)=D(b),
$$

which is also abelian. In addition, as shown in the proof (1) of Proposition $8.1 \theta_{J_{2}}$ has an extension to $C_{L_{+}}\left(R_{2}\right)$.

Let $\theta_{J}$ and $\theta$ be canonical characters of $b_{J}$ and $b$, respectively, so that $\theta_{J}=\theta_{J_{1}} \times \theta_{J_{2}}$ covers $\theta, \theta_{J}^{y}=\theta_{J}$ and $\theta \in \operatorname{Irr}\left(C_{K}(R) \mid \theta_{1} \times \theta_{2}\right)$. Applying Lemma 2.7 to

$$
L_{1} \times C_{L_{2}}\left(R_{2}\right) \leq C_{K}(R) \leq J_{1} \times C_{J_{2}}\left(R_{2}\right)
$$

we have that $\left.\theta_{J}\right|_{C_{K}(R)}=\theta$, so $\theta^{y}=\theta$ and $b^{y}=b$.

(2). If $q$ is even, then the outer-diagonal group of $K$ is trivial, so we may suppose $q$ is odd. Let $\left(P, g_{H}\right) \leq\left(R, b_{H}\right)$ be $B_{H}$-subgroups such that $g_{H}$ covers $g$ and $b_{H}$ covers $b$. Then $b_{H}$ is a block of $C_{H}(R)$ and $R=D\left(b_{H}\right) \cap C_{K}(R)$ for some $D\left(b_{H}\right)$. Since $H$ induces inner-diagonal automorphisms and since the outer-diagonal group of $K$ is a 2group, it follows that $D\left(b_{H}\right) \leq R Z(H)$ and $D\left(b_{H}\right)=R O_{p}(Z(H))$ is abelian. Similarly, $R=D(b)=D(g)=D\left(g_{H}\right) \cap C_{K}(P)$ and $D\left(g_{H}\right)=R O_{p}(Z(H))=D\left(b_{H}\right)$.

Now $z \in K \leq D_{0}(V)$, so by $[17,(2 \mathrm{E})], C_{D_{0}(V)}(z)=D_{0}\left(V_{0}\right) \circ \tilde{L}_{+}, C_{D_{0}(V)}(z) / Z_{+}=$ $C_{G}(z)$ and $C=C_{D_{0}(V)}(z) \cap K$, where $\tilde{L}_{+}$is a central extension of $L_{+}$by $Z_{+}$.

To show that $b_{H}^{y}=b_{H}$ we may suppose $H / K Z(H)=J_{0}(V) / K_{c} Z\left(J_{0}(V)\right)=$ $\operatorname{Outdiag}(K)$, so that $H / K Z(H)$ is a 2-group. Let $t \in C_{H}(z) \backslash C_{K}(z)$, so that $t^{4} \in$ $C_{K}(z) Z(H)$. In the notation of [20, Table 4.5.2], $t$ induces an element of $C^{*}:=$ $C_{\text {Inndiag }(K)}(z Z(K))$ (note here $C^{*}$ is not the dual group of $C$ ). But $C^{*} / C^{\text {o* }}$ is a $p$ group, so $t$ induce an element of $C^{\circ *}$ and hence $t \in C_{K}(z) Z(H)$. Thus

$$
C_{H}(z)=\left\langle C_{K}(z), x_{H}, t_{H}\right\rangle, \quad x_{H}=x_{1} x_{2}, \quad t_{H}=t_{1} t_{2}
$$

where $x_{1} \in D_{0}\left(V_{0}\right)$ and $x_{2} \in \tilde{L}_{+}$, and $t_{H}$ centralizes $\tilde{L}_{+}$. So

$$
\left\langle C_{K}(z), x_{H}\right\rangle \leq\left\langle J_{1}, x_{1}\right\rangle \circ\left\langle J_{2}, x_{2}\right\rangle \leq D_{0}\left(V_{0}\right) \circ \tilde{L}_{+} .
$$

Let $H_{1}=\left\langle J_{1}, x_{1}, t_{H}\right\rangle$ and $H_{2}=\left\langle J_{2}, x_{2}\right\rangle$, so that

$$
L_{1} \times L_{2} \leq C_{K}(z) \leq C_{H}(z) \leq H_{1} \circ H_{2} .
$$

It follows that

$$
L_{1} \times C_{L_{2}}\left(R_{2}\right) \leq C_{K}(R) \leq C_{H}(R) \leq H_{1} \circ C_{H_{2}}\left(R_{2}\right) .
$$


By [20, Table 4.5.2],

$$
\tilde{L}_{+}=\left(Z \times L_{2} \circ \mathbb{Z}_{q-\epsilon}\right)\left\langle x_{+}\right\rangle
$$

where $x_{+}$induces outer-diagonal automorphism of order dividing $\operatorname{gcd}(m, q-\epsilon)$ on $L_{2}$. Thus $C_{H_{2}}\left(R_{2}\right)=\left(Z \times C_{L_{2}}\left(R_{2}\right) \circ \mathbb{Z}_{q-\epsilon}\right)\left\langle y_{2}\right\rangle$ for some $y_{2} \in \tilde{L}_{+}$inducing outer-diagonal automorphism on $L_{2}$. View $\theta_{2}$ as character of $Z \times C_{L_{2}}\left(R_{2}\right)$ with $Z \leq \operatorname{ker}\left(\theta_{2}\right)$. Now $C_{L_{2}}\left(R_{2}\right) \leq C_{H_{2}}\left(R_{2}\right) / Z \leq C_{L_{+}}\left(R_{2}\right)$. As shown in the proof (1) of Proposition $8.1 \theta_{2}$ has an extension $\tilde{\theta}_{2}$ to $C_{H_{2}}\left(R_{2}\right) / Z$ such that $\tilde{\theta}_{2}^{y}=\tilde{\theta}_{2}$. By Remark $7.3 \theta_{H}^{y}=\theta_{H}$ and $b_{H}^{y}=b_{H}$. Thus the Property $7.1(a *)$ holds for $(R, b)$.

Suppose $B_{2}$ satisfies Property $7.1(\mathrm{~d})$, so that $D\left(B_{2}\right)$ is abelian. Thus $D_{+}$and so $D(B)$ are abelian. Since $D(B)=D\left(B_{H}\right) \cap K$ for some $D\left(B_{H}\right)$, it follows that $D\left(B_{H}\right) \leq K Z(H)$ and $D\left(B_{H}\right)=D(B) O_{p}(Z(H))$ which is abelian.

Theorem 8.6 Let $K$ be a finite quasi-simple group of classical type over a field $\mathbb{F}_{q}$ and $B \in \operatorname{Blk}(K)$, and let $K \triangleleft H$ such that $H / K$ is abelian, $C_{H}(K) \leq Z(H)$, $H$ induces inner-diagonal automorphisms on $K$ and $B_{H} \in \operatorname{Blk}(H)$ covering $B$. If $p \mid q$, then either $D(B)=D\left(B_{H}\right)$ is cyclic or $l(B) \geq 2$. Suppose $p \nmid q$ and $p$ is odd. Then one of Properties $7.1(a *),(b),(c)$ and $(d)$ holds. In addition, if Property $7.1(b)$ or $(c)$ holds, then $p=3, K=\mathrm{SL}^{\eta}(3 d, q) / Z$ for some $Z \leq Z\left(\mathrm{SL}^{\eta}(3 d, q)\right)$ with $\operatorname{gcd}(6, d)=1$ and $3 \|(q-\eta)$.

Proof: We will follow the notation of [20]. In particular, $K_{u}$ denotes the universal group with the same type as $K$. If $p \mid q$ and $D(B)$ is noncyclic, then $D(B)$ is a Sylow subgroup of $K$ and $l(B)=l\left(B_{0}\right)$ with principal $B_{0}:=B_{0}(K) \in \operatorname{Blk}(K)$. But $B_{0}$ dominates the principal block $\bar{B}$ of $K / Z(K)=K_{a}$ and $l(\bar{B})+1$ is the number of $p^{\prime}$-conjugacy classes of $K_{a}$, so $l\left(B_{0}\right) \geq l(\bar{B}) \geq 2$. Suppose $p \nmid q$.

If $K=A_{n}^{\eta}(q)$, then set $\widehat{K}=K_{u}=\mathrm{SL}_{n+1}^{\eta}(q) \leq \mathrm{GL}_{n+1}^{\eta}(q)$, so that $K=\widehat{K} / Z$ for some $Z \leq Z\left(\mathrm{GL}_{n+1}^{\eta}(q)\right) \cap \widehat{K}$. We may take $\widehat{H} \leq \mathrm{GL}_{n+1}^{\eta}(q)$ such that $H=\widehat{H} / Z$.

If $K=B_{n}(q)=K_{a}=\Omega_{2 n+1}(q)$, then set $\widehat{K}=\Omega_{2 n+1}(q) \leq \widehat{H} \leq \mathrm{SO}_{2 n+1}(q)$ such that $H=\widehat{H} / Z$. If $K=B_{n}(q)=K_{u}=\operatorname{Spin}_{2 n+1}(q)=\operatorname{Spin}(V)$, then take $K=\widehat{K} \triangleleft \widehat{H}=H$ such that $H / Z(K) \leq \mathrm{SO}(V)$.

If $K=C_{n}(q)$, then we may take $\widehat{K}=\operatorname{Sp}_{2 n}(q)=\operatorname{Sp}(V) \leq \hat{H} \leq J_{0}(V)$ such that $H=\widehat{H} / Z$.

Suppose $K=D_{n}^{\eta}(q)$ with $(n, \eta)=(2 k+1, \pm)$ or $(2 k,-)$. If $K=\Omega_{2 n}^{\eta}(q)=\Omega(V)$, then $K=\widehat{K} \triangleleft \widehat{H}=H \leq J_{0}(V)$. If $K=P \Omega_{2 n}^{\eta}(q)=P \Omega(V)$, then take $\widehat{K}=$ $\Omega_{2 n}^{\epsilon}(q) \leq \widehat{H} \leq J_{0}(V)$ such that $H=\widehat{H} / Z$. If $K=\operatorname{Spin}_{2 n}^{\eta}(q)=\operatorname{Spin}(V)$, then take $K=\widehat{K} \triangleleft \widehat{H}=H$ such that $H / Z(K) \leq J_{0}(V)$.

Suppose $K=D_{2 k}^{+}(q)$ with $q$ even. Then $K=H$ and we may take $\widehat{K}=\widehat{H}=H$. Suppose $K=D_{2 k}^{+}(q)$ with $q$ odd, so that $Z\left(K_{u}\right)=\left\{1, z, z_{s}, z_{c}\right\}$ and $K_{u} / Z=\Omega_{4 k}^{+}(q)$, where $Z=\langle z\rangle$. If $K=\Omega_{4 k}^{+}(q)=\Omega(V)$, then take $\widehat{K}=K \leq \widehat{H} \leq J_{0}(V)$. If $K=P \Omega_{4 k}^{+}(q)=P \Omega(V)$, then take $\widehat{K}=\Omega(V) \leq \widehat{H} \leq J_{0}(V)$ such that $\widehat{H} / Z=H$. If $K=\operatorname{Spin}_{4 k}^{+}(q) / Z^{\prime}$ for $Z^{\prime}=\left\langle z_{s}\right\rangle$ or $\left\langle z_{c}\right\rangle$, then we may take $\widehat{K}=\operatorname{Spin}_{4 k}^{+}(q)=\operatorname{Spin}(V) \leq$ $\widehat{H} \leq D_{0}(V)$ such that $H=\widehat{H} / Z^{\prime}$. If $K=\operatorname{Spin}_{4 k}^{+}(q)=\operatorname{Spin}(V)$, then take $\widehat{K}=K$ and $\widehat{H}=H$. 
Let $\widehat{B} \in \operatorname{Blk}(\widehat{K})$ dominating $B$ and $\widehat{B}_{H} \in \operatorname{Blk}(\widehat{H})$ dominating $B_{H}$, so that $\widehat{B}_{H}$ covers $\widehat{B}$. By Propositions 8.1, 8.2, 8.3 and 8.5, one of Properties 7.1 (a*), (b) of (d) holds for $\widehat{B}$.

If Property $7.1(a *)$ holds for $\widehat{B}$, then there exist $\widehat{B}$-subgroups $(\widehat{P}, \hat{g}) \leq(\widehat{R}, \hat{b})$ satisfying Property $7.1(a *)$. By Lemma 7.6, Property $7.1(a *)$ holds for some $B$ subgroups $(P, g) \leq(R, b)$.

Suppose Property 7.1 (b) holds for $\widehat{B}$. By Proposition 8.1, $\widehat{K}=\mathrm{SL}_{n+1}^{\eta}(q), D(\widehat{B})=$ $3_{+}^{1+2}$ and $n+1=3 d$ with $\operatorname{gcd}(6, d)=1$ and $3 \|(q-\eta)$. In particular, $Z(D(\widehat{B}))=$ $O_{3}(Z(\widehat{K}))$ and we may suppose $D(B) \leq D(\widehat{B}) Z / Z$. If $O_{3}(Z(\widehat{K})) \leq Z$, then $D(B)=$ $\mathbb{Z}_{3}^{2}, D\left(B_{H}\right)=3^{2}$ or $\mathbb{Z}_{3} \prec \mathbb{Z}_{3} / O_{3}(Z(\widehat{K})) \cong 3_{+}^{1+2}$ and (c) holds. If $Z$ is a $3^{\prime}$-group, then $D(B)=D(\widehat{B})$ and $(\mathrm{b})$ holds.

If Property 7.1 (d) holds for $\widehat{B}$, then $D(\widehat{B})$ and $D\left(\widehat{B}_{H}\right)$ are both abelian. Since $Z \leq Z(\widehat{H}) \cap \widehat{K}$, it follows that $D(B)=D(\widehat{B}) Z / Z$ and $D\left(B_{H}\right)=D\left(\widehat{B}_{H}\right) Z / Z$, and so $D(B)$ and $D\left(B_{H}\right)$ are both abelian.

\section{$9 \quad$ Exceptional groups}

Suppose $p$ is odd. We will follow the notation of [20]. In this section we demonstrate that every nilpotent block of an exceptional group of Lie type has abelian defect groups. We first prove a simple lemma.

Lemma 9.1 Let $J_{i}$ be a finite group and $P_{i}$ a p-subgroup of $J_{i}$ such that $C_{J_{i} / Z_{i}}\left(P_{i} / Z_{i}\right)=$ $C_{J_{i}}\left(P_{i}\right) / Z_{i}$ for $i=1,2$, where $Z_{i}=O_{p}\left(Z\left(J_{i}\right)\right)$. Let $J=J_{1} \times J_{2}, P=P_{1} \times P_{2}$ and $Z \leq O_{p}(Z(J))$. Then

$$
C_{J}(P) / Z=C_{J / Z}(P / Z)
$$

Proof: Let $Z_{+}=Z_{1} \times Z_{2}=O_{p}(Z(J))$. Then

$C_{J / Z_{+}}\left(P / Z_{+}\right)=C_{J_{1} / Z_{1}}\left(P_{1} / Z_{1}\right) \times C_{J_{1} / Z_{1}}\left(P_{1} / Z_{1}\right)=C_{J_{1}}\left(P_{1}\right) / Z_{1} \times C_{J_{2}}\left(P_{2}\right) / Z_{2}=C_{J}(P) / Z_{+}$.

It is clear that $C_{J}(P) / Z \leq C_{J / Z}(P / Z)$. If $x Z \in C_{J / Z}(P / Z)$ for some $x \in J$, then $x Z_{+} \in C_{J / Z_{+}}\left(P / Z_{+}\right)$and so $x Z_{+}=\left(x_{1}, x_{2}\right) Z_{+}$for some $x_{i} \in C_{J_{i}}\left(P_{i}\right)$. In particular, $x=\left(x_{1}, x_{2}\right) x_{+}$for some $x_{+} \in Z_{+}$. Since $Z_{+} \leq C_{J}(P)$, it follows that $x \in C_{J}(P)$ and hence $C_{J / Z}(P / Z)=C_{J}(P) / Z$.

The lemma will be applied to a central product $J_{1} \circ J_{2}$ as $J_{1} \circ J_{2}=\left(J_{1} \times J_{2}\right) / Z_{0}$ for some $Z_{0} \leq Z\left(J_{1}\right) \cap Z\left(J_{2}\right)$.

Theorem 9.2 Let $K$ be a finite quasi-simple group of exceptional type over a field $\mathbb{F}_{q}$, let $B \in \operatorname{Blk}(K)$, and let $K \triangleleft H$ such that $C_{H}(K) \leq Z(H), H / K$ is cyclic, and $H$ induces inner-diagonal automorphisms on $K$. Let $B_{H} \in \operatorname{Blk}(H)$ be a block covering $B$. Choose (as we may) defect groups $D(B)$ and $D\left(B_{H}\right)$ of $B$ and $B_{H}$ respectively such that $D(B)=D\left(B_{H}\right) \cap K$. If $p \mid q$, then either $D(B)=D\left(B_{H}\right)$ is cyclic or $l(B) \geq 2$. If $p \nmid q$ and $p$ is odd, then one of the Properties $7.1(a),(b)$ and $(d)$ holds. 
Proof: If $p \mid q$, then a proof similar to that of Theorem 8.6 shows that either $D(B)=D\left(B_{H}\right)$ is cyclic or $l(B) \geq 2$.

Suppose $p \nmid q$. Let $K_{u}$ be the universal group, so that $K=K_{u} / Z$ for some $Z \leq Z\left(K_{u}\right)$. Since $Z\left(K_{u}\right)$ is cyclic of order 1,2 or 3 , it follows that $H$ induces the trivial action on $Z\left(K_{u}\right)$.

Before beginning the proof proper we introduce some notation.

Write $D:=D(B)$. If $Z(K) \neq \Omega_{1}(Z(D))$, then take $z \in Z(D) \backslash Z(K)$ with $|z|=p$. If $Z(K)=\Omega_{1}\left(Z(D)\right.$ ) (so in particular $p=3$ ), then take $z \in D$ such that $|z|=p^{2}$ and $z Z(K) \in Z(D / Z(K))$. Let $\left(z, B_{z}\right)$ be a $B$-subsection, which we choose to be major in the case $z \in Z(D)$ (that such a major subsection exists is $[2,4.15]$ ). In the case that $z \in Z(D)$, we may further choose $\left(z, B_{z}\right)$ so that $B_{z}$ (a block of $C:=C_{K}(z)$ ) has defect group D. By [20, Theorem 4.2.2] $C=O^{r^{\prime}}(C) T$, where $O^{r^{\prime}}(C)$ is a central product

$$
O^{r^{\prime}}(C)=L_{1} \circ L_{2} \circ \cdots \circ L_{\ell}
$$

with each $L_{i} \in \mathcal{L} i e(r)$, and $T$ is an abelian $r^{\prime}$-group inducing inner-diagonal automorphisms on each $L_{i}$. In general, it may be the case that $z \notin O^{r^{\prime}}(C)$. We introduce some more notation as follows to allow for this inconvenience: If $Z(C) \leq O^{r^{\prime}}(C)$, then define $s:=\ell$ and $L:=O^{r^{\prime}}(C)$. If $Z(C) \not \leq O^{r^{\prime}}(C)$, then define $s=\ell+1, L_{s}=Z(C)$ and

$$
L:=L_{1} \circ L_{2} \circ \cdots \circ L_{s}
$$

In all cases $C=L T, z \in L$ and $L \triangleleft C$. Let $B_{L}$ be a block of $L$ covered by $B_{z}$. There are uniquely defined blocks $B_{i} \in \operatorname{Blk}\left(L_{i}\right)$ such that if $\chi \in \operatorname{Irr}\left(B_{L}\right)$ with $\chi=\chi_{1} \circ \cdots \circ \chi_{s}$ for some $\chi_{i} \in \operatorname{Irr}\left(L_{i}\right)$, then $\chi_{i} \in \operatorname{Irr}\left(B_{i}\right)$. We write

$$
B_{L}=B_{1} \circ B_{2} \circ \cdots \circ B_{s}
$$

Each element $t \in T$ has the form $t_{1} t_{2} \cdots t_{s} t^{\prime}$, where $t^{\prime}$ centralizes $L$ and $t_{i}$ induces an inner-diagonal automorphism on $L_{i}$ and $\left[L_{i}, t_{j}\right]=1$ for $i \neq j$. Let $T^{\prime}=\left\langle t^{\prime}: t=\right.$ $\left.t_{1} t_{2} \cdots t_{s} \circ t^{\prime} \in T\right\rangle$, and

$$
J_{i}:=\left\langle L_{i}, t_{i}: t=t_{1} t_{2} \cdots t_{s} t^{\prime} \in T\right\rangle, \text { and } J:=J_{1} \circ J_{2} \circ \cdots \circ J_{s} \circ T^{\prime} .
$$

Then $L T \triangleleft J$ and $T^{\prime}$ is abelian. Let $B_{J}$ be a block of $J$ covering $B_{z}$, so that $B_{J}$ covers $B_{L}$. Thus

$$
B_{J}=B_{J_{1}} \circ B_{J_{2}} \circ \cdots \circ B_{J_{s}} \circ B_{T^{\prime}},
$$

where $B_{J_{i}} \in \operatorname{Blk}\left(J_{i}\right)$ covering $B_{i}$ and $B_{T^{\prime}} \in \operatorname{Blk}\left(T^{\prime}\right)$. Note that if $C_{J_{i}}\left(L_{i}\right) \leq Z\left(L_{i}\right)$ for all $i$, then the central product $J$ is over a subgroup of $Z(L)$.

Case 1. Suppose each $B_{i}$ satisfies Property $7.1(\mathrm{~d})$. Then each $D\left(B_{J_{i}}\right)$ is abelian and so is $D\left(B_{J}\right)$. Thus $D\left(B_{z}\right)=D\left(B_{J}\right) \cap C$ is abelian.

Case 2. Suppose $L$ is a direct product of $L_{i}$ 's, $C_{J_{i}}\left(L_{i}\right) \leq Z\left(L_{i}\right)$ for all $i$ and some $B_{j}$ satisfies Property $7.1(a *)$. Without loss of generality, take $j=1$. In addition, suppose each $L_{i}$ is classical and universal (or $L_{s}=Z(C)$ ). Thus

$$
L=L_{1} \times \cdots \times L_{s} \leq C \triangleleft J=J_{1} \times \cdots \times J_{s} \times T^{\prime} .
$$


We now define $R_{i}$. If $L_{i}=\mathrm{SL}^{\eta}\left(V_{i}\right)$, then denote $G_{i}=\operatorname{GL}^{\eta}\left(V_{i}\right)$ and let $B_{G_{i}} \in \operatorname{Blk}\left(G_{i}\right)$ be a weakly regular cover of $B_{J_{i}}$ and $R_{i}:=A\left(D\left(B_{G_{i}}\right)\right) \cap L_{i}$. If $L_{i}$ is not linear and unitary, then set $R_{i}=A\left(D\left(B_{i}\right)\right)$. In addition, let $\left(R_{i}, b_{i}\right)$ be a $B_{i}$-subgroup, and note that $\left(R_{i}, b_{i}\right)$ is a Sylow $B_{i}$-subgroup when $D\left(B_{i}\right)$ is abelian. Let

$$
R:=R_{1} \times \cdots \times R_{s} \leq L, \quad b_{L}:=b_{1} \times \cdots \times b_{s}
$$

so that $\left(R, b_{L}\right)$ is a $B_{L}$-subgroup. Since $z \in O_{p}(Z(L))$ and $R$ is abelian, it follows that $z \in R$ and $R \leq C$. By Propositions 8.1, 8.2, 8.3 and 8.5, each defect group $D\left(b_{i}\right)$ of $b_{i}$ is abelian. Let $\left(R, b_{z}\right)$ be a $B_{z}$-subgroup such that $b_{z}$ covers $b_{L}$, and $\left(R, b_{J}\right)$ be a $B_{J}$-subgroup such that $b_{J}$ covers $b_{z}$, so that $b_{J}$ covers $b_{L}$ and

$$
b_{J}=b_{J_{1}} \times \cdots \times b_{J_{s}} \times B_{T^{\prime}}
$$

where each $b_{J_{i}}$ covers $b_{i}$. By Propositions 8.1, 8.2, 8.3 and 8.5 again, each defect group $D\left(b_{J_{i}}\right)$ is abelian, so defect groups $D\left(b_{J}\right)$ and $D\left(b_{z}\right)$ of $b_{J}$ and $b_{z}$ respectively are both abelian, since we may suppose $D\left(b_{z}\right)=D\left(b_{J}\right) \cap C$. Note in the proof above that $\left(R, b_{z}\right)$ can be any $B_{z}$-subgroup such that $b_{z}$ covers $b_{L}$. Later we will choose a special such $B_{z}$-subgroup.

Suppose further that $B_{1}$ satisfies Property $7.1(a *)$ in Propositions 8.1, 8.2, 8.3 or 8.5 for $B_{1}$-subgroups $\left(P_{1}, g_{1}\right) \leq\left(R_{1}, b_{1}\right)$. Let

$L_{+}=\prod_{i=2}^{s} L_{i}, \quad R_{+}:=\prod_{i=2}^{s} R_{i}, \quad P=P_{1} \times R_{+}, \quad g_{L}=g_{1} \times\left(\prod_{i=2}^{s} b_{i}\right), \quad J_{+}=\left(\prod_{i=2}^{s} J_{i}\right) \times T^{\prime}$,

so that $C_{L}(R)=C_{L_{1}}\left(R_{1}\right) \times C_{L_{+}}\left(R_{+}\right) \leq C_{C}(R) \leq C_{J_{1}}\left(R_{1}\right) \times C_{J_{+}}\left(R_{+}\right)$, and $\left(P, g_{L}\right) \leq$ $\left(R, b_{L}\right)$. Since $B_{z}$ covers $B_{L}$, it follows that there exist $B_{z}$-subgroups $\left(P, g_{z}\right) \leq\left(R, b_{z}\right)$ such that $g_{z}$ covers $g_{L}$ and $b_{z}$ covers $b_{L}$. Let $\left(P, g_{J}\right) \leq\left(R, b_{J}\right)$ be $B_{J}$-subgroups such that $g_{J}$ covers $g_{z}$ and $b_{J}$ covers $b_{z}$. Thus $g_{J}$ covers $g_{L}, b_{J}$ covers $b_{L}$ and $g_{J}=g_{J_{1}} \times$ $\cdots \times g_{J_{s}} \times B_{T^{\prime}}$. In particular, $g_{J_{i}}=b_{J_{i}}$ for $i \geq 2$, where $b_{J_{i}}$ are given in (9.4). By Propositions 8.1, 8.2, 8.3 and 8.5 again, each $D\left(b_{J_{i}}\right)$ is abelian and $D\left(g_{J_{1}}\right)=D\left(b_{J_{1}}\right)$, and hence $D\left(g_{J}\right)=D\left(b_{J}\right)$ is abelian and $D\left(g_{z}\right)=D\left(g_{J}\right) \cap C=D\left(b_{J}\right) \cap C=D\left(b_{z}\right)$.

Let $\theta_{i}$ be the canonical character of $b_{i}$ and $\theta_{+}=\prod_{i \geq 2} \theta_{i}$, so that $\theta:=\theta_{1} \times \theta_{+}$is the canonical character of $b_{L}$ and the canonical character $\theta_{z}$ of $b_{z} \operatorname{covers} \theta$.

Since $\left(P_{1}, g_{1}\right) \leq\left(R_{1}, b_{1}\right)$ satisfy Property $7.1(a *)$, it follows that there exists $y \in$ $N_{C_{L_{1}}\left(P_{1}\right)}\left(R_{1}, b_{1}\right) \backslash C_{L_{1}}\left(R_{1}\right)$ such that $y^{4}=1, y^{2} \in C_{L_{1}}\left(R_{1}\right)$ and $[y, x] \notin Z\left(L_{1}\right)$ for some $x \in R_{1}$. Moreover, there exist subgroups $N_{i} \triangleleft M_{i}$ of $J_{1}$, and character $\phi_{i} \in \operatorname{Irr}\left(N_{i}\right)$ for $i=1,2$ such that $M_{i} / N_{i}$ is abelian,

$$
Z\left(L_{1}\right) \leq N_{1} \times N_{2} \leq C_{L_{1}}\left(R_{1}\right) \leq C_{J_{1}}\left(R_{1}\right) \leq M_{1} \circ M_{2}
$$

$\theta_{1}$ covers $\phi_{1} \times \phi_{2}, Z \cap N_{2}=1, \phi_{2}$ has a $y$-stable extension $\tilde{\phi}_{2}$ to $M_{2}$ and $[y, x]=1$ or in $Z N_{2}$ according as $x \in M_{1}$ or $M_{2}$, where $Z \leq Z\left(M_{1}\right) \cap Z\left(M_{2}\right)$ such that $M_{1} \circ M_{2}$ is the central product over $Z$.

Let $N_{2}^{\prime}=N_{1} \times C_{L_{+}}\left(R_{+}\right), M_{2}^{\prime}:=M_{1} \times C_{J_{+}}\left(R_{+}\right)$, and $\phi_{2}^{\prime}=\phi_{1} \times \theta_{+}$. Then $M_{2} / N_{2}$ and $M_{2}^{\prime} / N_{2}^{\prime}$ are abelian,

$$
Z(C) \leq N_{2}^{\prime} \times N_{2} \leq C_{C}(R) \leq C_{J}(R) \leq M_{2}^{\prime} \circ M_{2}
$$


$\phi_{2}$ has an extension $\tilde{\phi}_{2}$ to $M_{2}$ which is $y$-invariant, $[y, x]=1$ for any $x \in M_{2}^{\prime},[y, x] \in$ $Z N_{2}$ for any $x \in M_{2}, M_{2} \circ M_{2}^{\prime}$ is a central product over $Z$ and $\theta_{z} \in \operatorname{Irr}\left(C_{C}(R) \mid \phi_{2}^{\prime} \times \phi_{2}\right)$. By Remark 7.3, $\theta_{z}^{y}=\theta_{z}$, and so $b_{z}^{y}=b_{z}$. If $[y, x] \in Z(C)$ for all $x \in R$, then $[y, x] \in Z(C) \cap L_{1}=Z\left(L_{1}\right)$ for all $x \in R_{1}$, which is impossible. Thus $[y, x] \notin Z(C)$ for some $x \in R$ and Property $7.1(a *)$ holds for $\left(P, g_{z}\right) \leq\left(R, b_{z}\right)$ (with $\left.H:=K\right)$.

Case 3. Suppose that $K:={ }^{2} B\left(2^{2 a+1}\right),{ }^{2} G_{2}\left(3^{2 a+1}\right),{ }^{2} F_{4}\left(2^{2 a+1}\right), G_{2}(q),{ }^{3} D_{4}(q)$, $F_{4}(q)$ or $E_{6}^{-\epsilon}(q)$ with $q \equiv \epsilon \bmod 3$, and $B \in \operatorname{Blk}(K)$. Then $B$ satisfies one of Property $7.1(a *),(b)$ or $(d)$.

In each case $K=K_{u}$ and $z$ induces an inner automorphism on $K$, so it follows that each $L_{i}$ is a classical group (or possibly $L_{s}$ is abelian). Hence by the results of Section 8 each $B_{i}$ satisfies one of Property $7.1(a *)$, (b), (c) or (d).

Case 3.1. Suppose $B_{i}$ satisfies either Property 7.1 (b) or (c) for some $i$. Without loss of generality, take $i=1$. By Theorem 8.6, $p=3, L_{1}=\operatorname{SL}^{\epsilon_{1}}\left(3 d_{1}, q_{1}\right) / Z$ for some $Z \leq Z\left(\mathrm{SL}^{\epsilon_{1}}\left(3 d_{1}, q_{1}\right)\right), \operatorname{gcd}\left(6, d_{1}\right)=1$ and $3 \|\left(q_{1}-\epsilon_{1}\right)$. By [20, Table 4.7.3A], $\left(q_{1}, \epsilon_{1}\right)=(q, \epsilon)$ or $\left(q^{2}, 1\right)$ and $(K, C)$ are given in Table 2 , where $L_{\epsilon}:=\mathrm{SL}_{3}^{\epsilon}(q)$.

\begin{tabular}{|l|l|l|l|}
\hline $\mathrm{K}$ & $C$ & $K$ & $C$ \\
\hline${ }^{3} D_{4}(q)$ & $\left(\mathbb{Z}_{q^{2}+\epsilon q+1} \circ L_{\epsilon}\right) \cdot 3$ & $G_{2}(q)$ & $L_{\epsilon}$ \\
${ }^{2} F_{4}\left(2^{2 m+1}\right)$ & $\mathrm{SU}_{3}\left(2^{2 m+1}\right)$ & $F_{4}(q)$ & $\left(L_{\epsilon} \circ L_{\epsilon}\right) \cdot(3: 3)$ \\
$E_{6}^{-\epsilon}(q)$ & $\left(L_{\epsilon} \circ \mathrm{SL}_{3}\left(q^{2}\right)\right) \cdot(3: 3)$ & $E_{6}^{\epsilon}(q)_{u}$ & $\left(L_{\epsilon} \times L_{\epsilon} \circ L_{\epsilon}\right) \cdot(3: 3: 3)$ \\
$E_{7}(q)_{u}$ & $\left(L_{\epsilon} \circ \mathrm{SL}_{6}^{\epsilon}(q)\right) \cdot(3: 3)$ & $E_{8}(q)$ & $\left(E_{6}^{\epsilon}(q)_{u} \circ L_{\epsilon}\right) \cdot(3: 3)$ \\
\hline
\end{tabular}

Table 2: Possible $(K, C)$ with some $B_{i}$ satisfying Property 7.1 (b) or (c)

Case 3.1.1. If $K=G_{2}(q)$ or ${ }^{2} F_{4}\left(2^{2 m+1}\right)$, then $s=1$ and $L=C$ and $B_{z}=B_{L}=$ $B_{0}(L)$, so $B=B_{0}(K)$ with $D(B)=3_{+}^{1+2}$. In particular, $l(B) \geq 2$.

Case 3.1.2. Let $K={ }^{3} D_{4}(q)$, so that $C=\mathbb{Z}_{\frac{1}{3}\left(q^{2}+\epsilon q+1\right)} \times H_{\epsilon}$, where $H_{\epsilon}=\left\langle L_{\epsilon}, x\right\rangle$ with $x$ inducing outer-diagonal automorphism of order 3 on $L_{\epsilon}$. So $D\left(B_{z}\right)=\mathbb{Z}_{3} \backslash \mathbb{Z}_{3} \in$ $\operatorname{Syl}_{3}(C)$ and we may suppose $D\left(B_{z}\right) \in \operatorname{Syl}_{3}\left(G_{\epsilon}\right)$, where $G_{\epsilon}=\mathrm{GL}_{3}^{\epsilon}(q)$ contains $H_{\epsilon}$. Let $R_{\epsilon}=A\left(D\left(B_{z}\right)\right)=\left(\mathbb{Z}_{3}\right)^{3}$ and $P_{\epsilon}=\left\langle Z\left(L_{\epsilon}\right), \operatorname{diag}\{1, w, w\}\right\rangle \leq R_{\epsilon}$ such that $|w|=3$ in $\mathbb{F}_{q^{2}}^{\times}$. Then $C_{G_{\epsilon}}\left(P_{\epsilon}\right)=\mathbb{Z}_{q-\epsilon} \times \mathrm{GL}_{2}^{\epsilon}(q)$ and $C_{G_{\epsilon}}\left(R_{\epsilon}\right)=\left(\mathbb{Z}_{q-\epsilon}\right)^{3}$. Thus

$$
\mathbb{Z}_{\frac{1}{3}\left(q^{2}+\epsilon q+1\right)} \times C_{H_{\epsilon}}\left(R_{\epsilon}\right)=C_{C}\left(R_{\epsilon}\right) \leq C_{C}\left(P_{\epsilon}\right)=\mathbb{Z}_{\frac{1}{3}\left(q^{2}+\epsilon q+1\right)} \times C_{H_{\epsilon}}\left(P_{\epsilon}\right) .
$$

As shown in the proof of Proposition 8.1 the $B_{z}$-subgroups $\left(P_{\epsilon}, g\right) \leq\left(R_{\epsilon}, b\right)$ satisfy Property $7.1(a *)$.

Note that in the notation above $C_{G_{\epsilon}}\left(P_{\epsilon}\right) / Z=C_{G_{\epsilon} / Z}\left(P_{\epsilon} / Z\right)$ and $C_{G_{\epsilon}}\left(R_{\epsilon}\right) / Z=$ $C_{G_{\epsilon} / Z}\left(R_{\epsilon} / Z\right)$ for any $Z \leq Z\left(L_{\epsilon}\right)$. Let $b_{\epsilon} \in \operatorname{Blk}\left(H_{\epsilon}\right)$ and $B_{\epsilon} \in \operatorname{Blk}\left(G_{\epsilon}\right)$ covering $b_{\epsilon}$, so that $D\left(b_{\epsilon}\right)=D\left(B_{\epsilon}\right) \cap H_{\epsilon}=D\left(B_{\epsilon}\right)$. Thus $D\left(b_{\epsilon}\right) \in \operatorname{Syl}_{3}\left(C_{G_{\epsilon}}(t)\right)$ for some semisimple $3^{\prime}$-element $t$. In particular, $D\left(b_{\epsilon}\right)$ is either abelian with $\left|D\left(b_{\epsilon}\right)\right| \geq 9$ and $D\left(b_{\epsilon}\right) \not \leq L_{\epsilon}$ or $D\left(b_{\epsilon}\right)=\mathbb{Z}_{3} 2 \mathbb{Z}_{3}$. In the former case, $C_{G_{\epsilon}}\left(D\left(b_{\epsilon}\right)\right) / Z=C_{G_{\epsilon} / Z}\left(D\left(b_{\epsilon}\right) / Z\right)$ for any $Z \leq Z\left(L_{\epsilon}\right)$. 
Case 3.1.3. Suppose $K=E_{6}^{-\epsilon}(q)$ or $F_{4}(q)$ and $L_{1}=L_{\epsilon}$, so that $C=\left\langle L_{1} \circ L_{2}, x\right\rangle$, where $L_{2}=L_{\epsilon}$ or $\mathrm{SL}_{3}\left(q^{2}\right)$, and $x=x_{1} x_{2}$ such that each $x_{i}$ induces outer-diagonal automorphism of order 3 on $L_{i}$. Let $J_{i}=\left\langle L_{i}, x_{i}\right\rangle$ and $B_{J_{2}} \in \operatorname{Blk}\left(J_{2}\right)$ covering $B_{2}$. Let $R_{1}=R_{\epsilon} \leq J_{1}, P_{1}=P_{\epsilon} \leq R_{1}$ and $P_{2}=R_{2}=A\left(B_{J_{2}}\right)$, so that by the remark of Case 3.1.2 above, $C_{J_{i}}\left(P_{i}\right) / Z=C_{J_{i} / Z}\left(P_{i} / Z\right)$ and $C_{J_{i}}\left(R_{i}\right) / Z=C_{J_{i} / Z}\left(R_{i} / Z\right)$ for any $Z \leq Z\left(L_{i}\right)$. By Lemmas 9.1 and 7.6 , we may suppose

$$
L=L_{1} \times L_{2} \leq C \triangleleft J:=J_{1} \times J_{2}
$$

Let $R=\left(R_{1} \times R_{2}\right) \cap C, P=\left(P_{1} \times P_{2}\right) \cap C$ and let $(P, g) \leq(R, b)$ be $B_{z}$-subgroups, so that $\pi_{1}(P)=P_{\epsilon}$ and $\pi_{1}(R)=R_{\epsilon}$, where $\pi_{i}$ is the natural projection form $J$ to $J_{i}$. A proof similar to that of Case 2 shows that $(P, g) \leq(R, b)$ satisfy Property 7.1 ( $a *)$. If $K=E_{6}^{-\epsilon}(q)$ and $L_{1}=\mathrm{SL}_{3}\left(q^{2}\right)$, then $L_{2}=L_{\epsilon}$ and a similar proof shows that Property 7.1 ( $a *$ ) holds for some $B$-subgroups $(P, g) \leq(R, b)$.

Note that $C_{J}(P) / Z=C_{J / Z}(P / Z)$ and $C_{J}(R) / Z=C_{J / Z}(R / Z)$ for any $Z \leq O_{3}(Z(L))$.

Case 3.2. Suppose that each $B_{i}$ satisfies either Property $7.1(a *)$ or (d). By Case 1 , we may suppose, moreover that $B_{1}$ satisfies Property $7.1(a *)$. In particular, a Sylow $p$-subgroup of $L_{1}$ is nonabelian.

Case 3.2.1. Suppose $p \geq 5$, so that $z$ is of parabolic type. By [20, Theorem 4.2.2 (f)], $O^{r^{\prime}}(C)$ is a direct product and each $L_{i}$ is universal. In addition, if a Sylow $p$-subgroup of $L_{1}$ is nonabelian, then $\ell=1$ or 2 and each $L_{i}$ is universal.

Suppose $\ell=1$, so that $s=1$ or 2 . Since $B_{1}$ satisfies Property $7.1(a *)$ and $L_{s}=Z(C)$ when $s=2$, it follows by Lemma 9.1 that we may suppose $L=L_{1} \times L_{s}$ and $C_{J_{i}}\left(L_{i}\right) \leq Z\left(L_{i}\right)$.

Suppose $\ell=2$, so that $s=2$ or 3 . Since $L_{1}$ has a nonabelian Sylow $p$-subgroup, it follows that $L_{2}$ has an abelian Sylow $p$-subgroup and, moreover $L_{2} \cap O_{p}(Z(C))=1$. Since the central product $L_{1} \circ L_{2} \circ L_{s}$ is over a subgroup of $Z(C)$, it follows that each $p$-subgroup of $L_{2}$ satisfies the assumption of Lemma 9.1. Since $B_{1}$ satisfies Property $7.1(a *)$ and $L_{s}=Z(C)$ when $s=3$, it follows by Lemma 9.1 that we may suppose $L=L_{1} \times L_{s} \times L_{s}$ and $C_{J_{i}}\left(L_{i}\right) \leq Z\left(L_{i}\right)$.

By Case 2, $B$ satisfies Property $7.1(a *)$.

Case 3.2.2. Suppose $p=3$, so that $C$ is given by [20, Table 4.7.3a]. Thus either $\ell=1$ or $\ell=2$ with $C$ given by Table 2 . In addition, each $L_{i}$ is also universal for $1 \leq i \leq \ell$ and $C_{J_{i}}\left(L_{i}\right) \leq Z\left(L_{i}\right)$.

A proof similar to that of Case 3.2.1 shows that we may suppose (9.3) holds and by Case 2, $B$ satisfies Property $7.1(a *)$.

Case 4. Let $3 \mid(q-\epsilon), K=K_{u}=3 \cdot E_{6}^{\epsilon}(q) \leq E:=3 \cdot E_{6}^{\epsilon}(q) .3, B \in \operatorname{Blk}(K)$ and $B_{E} \in \operatorname{Blk}(E)$ covering $B$. Either Property $7.1(a *)$ holds for some $B$-subgroups $(P, g) \leq(R, b)$ with $C_{E}(P) / Z=C_{E / Z}(P / Z)$ and $C_{E}(R) / Z=C_{E / Z}(R / Z)$ for $Z \leq$ $O_{p}(K)$, or Property $7.1(d)$ holds for $B$.

Let $D:=D(B)$ and $m^{*}:=\operatorname{gcd}(m, q-\epsilon)$.

If some $B_{i}$ satisfies either Property 7.1 (b) or (c), then $p=3$ and $C$ is given by Table 2. A proof similar to that of Case 3.2.2 shows that Property 7.1 (a*) holds for some $B$-subgroups $(P, g) \leq(R, b)$.

Suppose some $B_{i}$ satisfies Property $7.1(a *)$. Since $z$ is parabolic or equal-rank type and $z$ induces an inner automorphism on $K$, it follows that each $L_{i}$ is classical. We 
first show that there exist $B$-subgroups $(P, g) \leq(R, b)$ and $y$ satisfying the Property $7.1(a *)$ with $H:=K$.

If $p \geq 5$, then $z$ is parabiloc. A proof similar to that of Case 3.2.1 shows that the Property $7.1(a *)$ holds for $B$-subgroups $(P, g) \leq(R, g)$.

Suppose $p=3$. By [20, Table 4.7.3A],

$$
C_{K}(z)=\left\langle\mathrm{SL}_{3}^{\epsilon}(q) \times \mathrm{SL}_{3}^{\epsilon}(q) \circ \mathrm{SL}_{3}^{\epsilon}(q), 3: 3: 3\right\rangle, \quad\left(\mathrm{SL}_{6}^{\epsilon}(q) \circ 2^{*}(q-\epsilon)\right) .2^{*}
$$

$\operatorname{Spin}_{8}^{+}(q) \circ_{2^{*}}((q-\epsilon) \times(q-\epsilon)) \cdot\left(2^{*} \times 2^{*}\right), \operatorname{Spin}_{10}^{\epsilon}(q) \circ(q-\epsilon) \cdot\left(2^{*} \times 2_{\epsilon}^{*}\right)($ when $q \equiv \epsilon$ $(\bmod 9))$ with $2_{\epsilon}^{*}=1$ or $2^{*}$ according as $\epsilon=-$ or + , or $\left(\mathrm{SL}_{2}(q) \times \mathrm{SL}_{5}^{\epsilon}(q)\right) \circ(q-\epsilon) .2^{*}$ (when $q \equiv \epsilon \quad(\bmod 9))$.

Thus $\ell=1,2$ or 3 . If $\ell=1$ or 2 , then a proof similar to that of Cases 3.2.1 and 3.2.2 shows that the Property $7.1(a *)$ holds for $B$-subgroups $(P, g) \leq(R, g)$. If $\ell=3$, then each $L_{i}=\mathrm{SL}_{3}^{\epsilon}(q)$ and $C_{J_{i}}\left(L_{i}\right) \leq Z\left(L_{i}\right)$. A proof similar to that of Cases 3.1.3 shows that we may suppose (9.3) holds and by Case 2, the Property $7.1(a *)$ holds for $B$-subgroups $(P, g) \leq(R, g)$.

If all $B_{i}$ satisfies Property $7.1(\mathrm{~d})$, then by Case $1, D\left(B_{z}\right)$ is abelian.

Now we prove the rest of Property $7.1(a *)$ with $H=E$. Suppose $\left(P, g_{E}\right) \leq\left(R, b_{E}\right)$ are $B_{E}$-subgroups such that $g_{E}$ covers $g$ and $b_{E}$ covering $b$.

Case 4.1. If $p \geq 5$, then $D(b)=D\left(b_{E}\right), D(g)=D\left(g_{E}\right)$, and so $D\left(g_{E}\right)=D\left(b_{E}\right)$ is abelian. Now $C_{E}(z)=C$ or $\langle C, x\rangle$ for some $x \in E \backslash K$. If $C_{E}(z)=C$, then $C_{E}(R)=C_{C}(R)$. Apply the proof of Case 2 we have that

$$
Z(C) \leq N_{2}^{\prime} \times N_{2} \leq C_{C}(R) \leq C_{C_{E}(z)}(R) \leq M_{2}^{\prime} \circ M_{2} .
$$

Suppose $C_{E}(z)=\langle L T, x\rangle$ for some $x \in E \backslash K$, so that $x$ induces inner-diagonal automorphism on each $L_{i}$. Thus $x=x_{1} x_{2} \cdots x_{s} x^{\prime}$. Replacing $J_{i}$ by $\left\langle J_{i}, x_{i}\right\rangle$ and $T^{\prime}$ by $\left\langle T^{\prime}, x^{\prime}\right\rangle$ in the proof Case 2 with some obvious modifications, we have that (9.5) still holds. Thus Property $7.1(a *)$ holds for $B$-subgroups $(P, g) \leq(R, b)$ (with $H:=E$ ).

Case 4.2. Suppose $p=3$. By [20, Table 4.7.3A],

$$
C_{E}(z)=\left\langle\mathrm{SL}_{3}^{\epsilon}(q) \times \mathrm{SL}_{3}^{\epsilon}(q) \circ \mathrm{SL}_{3}^{\epsilon}(q), 3: 3: 1,1: 3: 3\right\rangle, \quad\left(\mathrm{SL}_{6}^{\epsilon}(q) \circ_{2^{*}}(q-\epsilon)\right) \cdot\left(3 \times 2^{*}\right),
$$

$\operatorname{Spin}_{8}^{+}(q) \circ_{2^{*}}((q-\epsilon) \times(q-\epsilon)) \cdot\left(2^{*} \times 2^{*} \times 3\right), \operatorname{Spin}_{10}^{\epsilon}(q) \circ(q-\epsilon) \cdot\left(3 \times 2^{*} \times 2_{\epsilon}^{*}\right)($ when $q \equiv \epsilon$ $(\bmod 9))$ with $2_{\epsilon}^{*}=1$ or $2^{*}$ according as $\epsilon=-$ or + , or $\left(\mathrm{SL}_{2}(q) \times \mathrm{SL}_{5}^{\epsilon}(q)\right) \circ(q-\epsilon) \cdot\left(2^{*} \times 3\right)$ (when $q \equiv \epsilon \quad(\bmod 9))$.

Suppose $C_{E}(z)=\left\langle\mathrm{SL}_{3}^{\epsilon}(q) \times \mathrm{SL}_{3}^{\epsilon}(q) \circ \mathrm{SL}_{3}^{\epsilon}(q), t, x\right\rangle$, so that $L=\mathrm{SL}_{3}^{\epsilon}(q) \times \mathrm{SL}_{3}^{\epsilon}(q) \circ$ $\mathrm{SL}_{3}^{\epsilon}(q), T=\langle t\rangle \leq K$ with $t$ induces 3:3:3 on $L$, and $x \in E \backslash K$ induces 1:3:3 on $L$. Let $L_{i}=\mathrm{SL}_{3}^{\epsilon}(q) \leq G_{i}:=\mathrm{GL}_{3}^{\epsilon}(q), t=t_{1} \times t_{2} \times t_{3}, x=x_{1} \times x_{2} \times x_{3}$ with $t_{i}, x_{i}$ act on $L_{i}$ and centralizes $L_{j}$ when $i \neq j$. In addition, let $H_{i}=\left\langle L_{i}, t_{i}, x_{i}\right\rangle$, so that $H_{i} \leq G_{i}$. Let $S=\mathbb{Z}_{q-\epsilon} \times \mathbb{Z}_{q-\epsilon} \leq \mathrm{SL}_{3}^{\epsilon}(q)$ be a maximal torus, and $S \times S \circ_{3} S \leq L$. Since $C_{\mathrm{GL}_{3}^{\epsilon}(q)}(S)=\mathbb{Z}_{q-\epsilon} \times \mathbb{Z}_{q-\epsilon} \times \mathbb{Z}_{q-\epsilon}$ is a maximal torus, it follows that $A:=C_{E}\left(S \times S \circ_{3} S\right)$ is abelian such that $A \cap K \cong \mathbb{Z}_{q-\epsilon}^{6}$ is a maximal torus of $K$ and $A /(A \cap K)=\mathbb{Z}_{3}$. In particular, we may suppose $t, x \in A$ and $C_{E}(z)=L A$ with abelian $A$ and $L \triangleleft C_{E}(z)$.

Similarly, if $C_{E}(z)=\left(\operatorname{SL}_{6}^{\epsilon}(q) \times(q-\epsilon)\right) \cdot 6^{*}, \operatorname{Spin}_{8}^{+}(q) \circ_{2}((q-\epsilon) \times(q-\epsilon)) \cdot\left(2^{*} \times\right.$ $\left.6^{*}\right), \operatorname{Spin}_{10}^{\epsilon}(q) \circ(q-\epsilon) \cdot\left(6^{*} \times 2_{\epsilon}^{*}\right)$ or $\left(\mathrm{SL}_{2}(q) \times \mathrm{SL}_{5}^{\epsilon}(q)\right) \circ(q-\epsilon) \cdot 6^{*}$, then $A \leq C_{E}(z)$ and so $C_{E}(z)=L A$ with abelian $A$ and $L \triangleleft C_{E}(z)$, and $A$ induces inner-diagonal automorphisms on each $L_{i}$. 
A proof similar to that of Case 2 with $L T$ replaced by $L A$ and some modifications shows that $D\left(g_{E}\right)=D\left(b_{E}\right)$ is abelian, and hence Property $7.1(a *)$ holds.

Case 4.3. Suppose $p=3$ and $Z=Z(K)$. If $C_{E}(z) / Z=C_{E / Z}(z Z)$, then $C_{E}(P) / Z=$ $C_{E / Z}(P / Z)$ and $C_{E}(R) / Z=C_{E / Z}(R / Z)$. Suppose $C_{E}(z) / Z \neq C_{E / Z}(z Z)$. By [20, Table 4.7.3A], either $L=L_{1} \circ L_{2}$ with $L_{1}=\operatorname{Spin}_{8}^{+}(q)$ and $L_{2}=Z(C)=\mathbb{Z}_{q-\epsilon} \times \mathbb{Z}_{q-\epsilon}$ or $L=L_{\epsilon} \times L_{\epsilon} \circ L_{\epsilon} \leq K$. In the former case,

$$
C_{E / Z}(z Z)=\left\langle C_{E}(z) / Z, w Z\right\rangle
$$

where $w \in E$ such that $w=\gamma: \omega$, that is, $w$ acts on $L_{1}$ as a graph automorphism of order 3 and $\left(h_{1}, h_{2}\right)^{\omega}=\left(h_{2},\left(h_{1} h_{2}\right)^{-1}\right)$ for any $\left(h_{1}, h_{2}\right) \in L_{2}$. Now $O_{3}\left(L_{2}\right)=O_{3}\left(\mathbb{Z}_{q-\epsilon}\right) \times$ $O_{3}\left(\mathbb{Z}_{q-\epsilon}\right) \leq P$ and $Z(K)=\left\{(x, x): x \in \Omega_{1}\left(O_{3}\left(\mathbb{Z}_{q-\epsilon}\right)\right)\right\} \leq Z(C)$. Suppose $h \in$ $E$ such that for any $u \in P$ we have $h^{-1} u h=c u$ for some $c \in Z(K)$ and suppose $h \notin C_{E}(z)$. Then we may suppose $h=t w$ for some $t \in C_{E}(z)$, and so $\left(1, h_{2}\right)^{h}=$ $\left(1, h_{2}\right)^{\omega}=\left(h_{2}, 1\right)$ for any $h_{2} \in O_{3}\left(\mathbb{Z}_{q-\epsilon}\right) \backslash\{1\}$. But $\left(h_{2}, 1\right) \neq c\left(1, h_{2}\right)$ for any $c \in Z(K)$, which is a contradiction. Thus $h \in C_{E}(z)$ and so $C_{E}(P) / Z=C_{E / Z}(P / Z)$. Similarly, $C_{E}(R) / Z=C_{E / Z}(R / Z)$. If $L=L_{\epsilon} \times L_{\epsilon} \circ L_{\epsilon}$, then $L / Z=L_{\epsilon} \circ L_{\epsilon} \circ L_{\epsilon}$ and $C_{E / Z}(z Z)=$ $\langle L / Z, t Z, x Z, w Z\rangle$, where $t, x$ are given above and $w \in E \backslash K$ permutes transitively the three components $L_{\epsilon}$ of $L$. The proof in this case is similar. Suppose $h \in E$ such that for all $u \in P h^{-1} u h=c u$ for some $c \in Z$, so that $h Z \in C_{E / Z}(z Z)$. Since $\left|\Omega_{1}(P)\right| \geq 3^{4}$ and $C_{J}(P) / Z=C_{J / Z}(P / Z)$, it follows that $h \in C_{E}(z)=\langle L, x, t\rangle$ and hence $C_{E}(P) / Z=C_{J / Z}(P / Z)$. Similarly, $C_{E}(R) / Z=C_{E / Z}(R / Z)$.

Case 4.4. Now we prove the rest of Property 7.1 (d). Suppose Property $7.1(d)$ holds for each $B_{i}$ and suppose $D_{E} \cap K=D$ for some $D_{E}=D\left(B_{E}\right)$, so that $D\left(B_{J_{i}}\right)$ is abelian and so is $D\left(B_{z}\right)=D\left(B_{J}\right) \cap C$. If $B_{z}$ is a major subsection, then $D=D\left(B_{z}\right)$ is abelian. If $p \geq 5$, then $D_{E}=D$. Suppose $p=3$ and there exists $x \in Z\left(D_{E}\right) \backslash D$. Then $x \in E \backslash K, x \in C_{E}(D)$ and $D_{E}=\langle D, x\rangle$ is abelian. If $Z\left(D_{E}\right) \leq D$, then take $z \in Z\left(D_{E}\right)$ with $|z|=3$, so that $D_{E} \leq C_{E}(z)=L A$. A proof similar to that of Case 1.2 with some obvious modifications shows that $D_{E}$ is abelian.

Suppose $z \in D$ with $|z|=9$ and $z Z(K) \in Z(D / Z(K))$. By [20, Table 4.7.3A], $9 \mid(q-\epsilon)$ and $C_{E}(z)=\operatorname{Spin}_{10}^{\epsilon}(q) \circ(q-\epsilon) \cdot\left(6^{*} \times 2_{\epsilon}^{*}\right)$ or $\left(\mathrm{SL}_{2}(q) \times \mathrm{SL}_{5}^{\epsilon}(q)\right) \circ(q-$ $\epsilon) .6^{*}$. In this case, $C_{E / Z(K)}(z Z(K))$ is also given by [20, Table 4.7.3A], and we have $C_{K / Z(K)}(z Z(K))=C_{K}(z) / Z(K)$. Thus $D / Z(K) \leq C_{K}(z) / Z(K)$ and $D \leq C_{K}(z)$. In particular, $z \in Z(D)$ and we may suppose $\left(z, B_{z}\right)$ is major. Hence $D=D\left(B_{z}\right)$ is abelian. It follows that Property 7.1 (d) holds for $B$.

Case 5. Let $K:=E_{7}(q)$ with $q$ even and $B \in \operatorname{Blk}(K)$. Then either $L_{i}$ is classical, or $L_{i}$ is exceptional given in Cases 3 or 4 . If $L_{i}$ is classical, then apply Propositions 8.1, 8.2, 8.3 and 8.5. If $L_{i}$ is exceptional, then apply the results given in Cases 3 and 4. Either Property $7.1(a *)$ holds for $B$ or Property $7.1(d)$ holds for $B$ (with $\left.H:=K:=E_{7}(q)\right)$.

Let $q$ be odd, $K=2 . E_{7}(q) \leq E:=2 . E_{7}(q) .2, B \in \operatorname{Blk}(K)$ and $B_{E} \in \operatorname{Blk}(E)$ covering $B$. Either Property $7.1(a *)$ holds for some $B$-subgroups $(P, g) \leq(R, b)$ or Property $7.1(d)$ holds for $B$.

Again let $D:=D(B)$ and $m^{*}:=\operatorname{gcd}(m, q-\epsilon)$. 


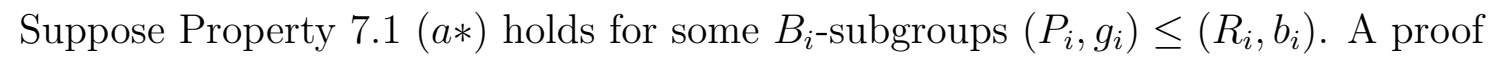
similar to that of Case 3.2 with some obvious modifications shows that there exist $B$-subgroups $(P, g) \leq(R, b)$ and $y$ satisfying the first part of Property $7.1(a *)$ (with $H=K)$. Suppose $B_{i}$ satisfies either Property 7.1 (b) or (c) for some $i$. Then $p=3$ and $C$ is given by Table 2. A proof similar to that of Case 3.1.3 shows that Property 7.1 (a*) holds for some $B$-subgroups $(P, g) \leq(R, b)$. Suppose $\left(P, g_{E}\right) \leq\left(R, b_{E}\right)$ are $B_{E}$-subgroups such that $g_{E}$ covering $g$ and $b_{E}$ covering $b$. Then $D(g)=D\left(g_{E}\right)$ and $D(b)=D\left(b_{E}\right)$ for some $D\left(g_{E}\right)$ and $D\left(b_{E}\right)$. But $D(g)=D(b)$ is abelian, so $D\left(g_{E}\right)=$ $D\left(b_{E}\right)$ is abelian. A proof similar to that of Case 4.1 shows that (9.5) holds and so Property $7.1(a *)$ (with $H=E$ ) holds for $B$-subgroups $(P, g) \leq(R, b)$.

Since $E / K=2$ and $p$ is odd, it follows that $D\left(B_{E}\right)$ is abelian whenever $D(B)$ is abelian.

Case 6. Suppose $K:=E_{8}(q)$. Either Property 7.1 (a) holds for some $B$-subgroups $(P, g) \leq(R, b)$ or Property $7.1(d)$ holds for $B$.

In this case $\left(z, B_{z}\right)$ is a major subsection of $B$ and either $L_{i}$ is classical, or $L_{i}$ is exceptional given in Cases 3,4 or 5. If $L_{i}$ is classical, then apply Theorem 8.6. If $L_{i}$ is exceptional, then apply the results in Cases 3,4 or 5 . Thus if each $D\left(B_{i}\right)$ is abelian, then $D\left(B_{J_{i}}\right)$ is abelian, and so $D=D\left(B_{z}\right)=D\left(B_{J}\right) \cap C$ is abelian. Suppose $D\left(B_{i}\right)$ is non-abelian for some $i$, say $i=1$.

If $p \geq 7$, then $B_{1}$ satisfies Property $7.1(a *), z$ is of parabolic type and the proof is similar to that of Case 3.2.1.

Suppose $p=5$, so that $B_{1}$ satisfies Property $7.1(a *)$ and $C$ is given by [20, Table 4.7.3B]. Thus $\ell=1$ or 2 . If $z$ is parabolic, then a proof similar to that of Case 3.2.1 shows that we may suppose (9.3) holds.

If $z$ is equal-rank, then

$$
C=\left\langle L_{1} \circ L_{2}, 5: 5\right\rangle, \quad L_{1}=L_{2}=\mathrm{SL}_{5}^{\epsilon}(q),
$$

so that $L=L_{1} \circ L_{2}$. Here $\epsilon= \pm 1$ such that $q \equiv \epsilon(\bmod 5)$. A proof similar to that of Case 3.1.3 shows that we may suppose $L=L_{1} \times L_{2}$. By Case 2, $B$ satisfies Property $7.1(a *)$.

Suppose $p=3$, so that $C$ is given by [20, Table 4.7.3A] and $\ell=1$ or 2 .

If some $B_{i}$ satisfies either Property 7.1 (b) or (c), then $C$ is given by Table 2 . In particular, $\ell=2$ and $D\left(B_{i}\right)=3_{+}^{1+2}$.

If $\ell=1$, then $B_{1}$ satisfies Property $7.1(a *)$ and we may suppose (9.3) holds. By Case 2, $B$ satisfies Property $7.1(a *)$.

Suppose $\ell=2$, so that $L=L_{\epsilon} \circ E_{6}(q)_{u}$ and $C=\langle L, 3: 3\rangle$, where $L_{\epsilon}=\mathrm{SL}_{3}^{\epsilon}(q)$ with $q \equiv \epsilon \quad(\bmod 3)$.

If $L_{1}=E_{6}(q)_{u}$ and $B_{1}$ satisfies Property $7.1(a *)$ for $\left(P_{1}, g_{1}\right) \leq\left(R_{1}, b_{1}\right)$. Let $P_{2}=R_{2}=A\left(D\left(B_{2}\right)\right)$, and let $\left(R_{2}, b_{2}\right)$ be a $B_{2}$-subgroup and set $\left(P_{2}, g_{2}\right)=\left(R_{2}, b_{2}\right)$. By Case 4 and the ramark of Case 3.1.2, $C_{J_{i}}\left(R_{i}\right) / Z_{i}=C_{J_{i} / Z_{i}}\left(R_{i} / Z_{i}\right)$ for $i=1,2$. By Lemma 9.1, we may suppose $L=L_{1} \times L_{2}$ and a similar to that of Case 2 shows that $B$ satisfies Property $7.1(a *)$.

Suppose $L_{1}=L_{\epsilon}$ and $B_{2}$ satisfies Property $7.1(\mathrm{~d})$, so that $D\left(B_{2}\right)$ is abelian. In this case $B_{1}$ satisfies Property 7.1 (b) with $D\left(B_{1}\right)=3_{+}^{1+2}$ or $B_{1}$ satisfies Property 7.1 $\left(\mathrm{a}^{*}\right)$. Note that $J=J_{1} \circ J_{2}$ over $Z(L)=3$ and $J_{1}=H_{\epsilon}$ given in Case 3.1.2. 
Let $\left(P_{\epsilon}, g\right) \leq\left(R_{\epsilon}, b\right)$ be defined in Case 3.1.2. Then there exists $y \in C_{H_{\epsilon}}\left(P_{\epsilon}\right) \cap$ $N_{H_{\epsilon}}\left(R_{\epsilon}\right) \backslash C_{H_{\epsilon}}\left(R_{\epsilon}\right)$ satisfies Property $7.1(a *)$.

Let $P_{2}=R_{2}=A\left(D\left(B_{J_{2}}\right)\right)=D\left(B_{2}\right)$ and let $\left(P_{2}, b_{2}\right)=\left(R_{2}, b_{2}\right)$ be the Sylow $B_{J_{2}}$-subgroup. Since $H_{\epsilon}=J_{1}$, it follows that $P_{\epsilon} \circ P_{2} \leq R_{\epsilon} \circ R_{2} \leq J$. Set

$$
P=\left(P_{\epsilon} \circ P_{2}\right) \cap C, \quad \text { and } \quad R=\left(R_{\epsilon} \circ R_{2}\right) \cap C .
$$

Then $P \leq R \leq D$ and $P, R$ are abelian. Since $Z(L)=3$, it follows that $b \circ b_{2}$ is a block $C_{J}\left(R_{\epsilon} \circ R_{2}\right)$ and $g \circ g_{2} \in \operatorname{Blk}\left(C_{J}\left(P_{\epsilon} \circ P_{2}\right)\right)$. Since $J / C=3$, it follows that $b \circ b_{2}$ covers a unique block $b_{R}$ of $C_{C}(R)$ and similarly, $g \circ g_{2}$ covers a unique block $g_{P}$ of $C_{C}(P)$. Since $B_{J}$ is the unique block covering $B_{z}$, it follows that $\left(P, b_{P}\right) \leq\left(R, b_{R}\right)$ are $B_{z}$-subgroups. Since $H_{\epsilon} / L_{\epsilon}=3$, it follows that $y \in L_{\epsilon}=L_{1}$. Now $B$ satisfies Property 7.1 (a) (not $(a *))$ for $B$-subgroups $\left(P, b_{P}\right) \leq\left(R, b_{R}\right)$ (with $\left.H=K\right)$.

Lemma 9.3 Let $G$ be a quasisimple group such that $G / Z(G)$ is alternating or of Lie type and $G$ is an exceptional cover. Let $p$ be an odd prime. Then every p-block of $G$ with nonabelian defect groups has a subpair with at least two irreducible Brauer characters.

Proof: We must consider the cases $G / Z(G) \cong P S L_{2}(4), P S L_{2}(9), A_{7}, P S L_{3}(2)$, $P S L_{3}(4), P S U_{4}(2), P S U_{4}(3), P S U_{6}(2),{ }^{2} B_{2}(8), O_{7}(2), O_{7}(3), O_{8}^{+}(2), G_{2}(3), G_{2}(4)$, $F_{4}(2)$ and ${ }^{2} E_{6}(2)$. We may use [18] to confirm all but the cases $F_{4}(2)$ for $p=3$, and ${ }^{2} E_{6}(2)$ for $p=3,5,7$ (noting that the three double covers of $O_{8}^{+}(2)$ have the same block structure - see [14]), as in each case the block itself has at least two irreducible Brauer characters. The result holds for $F_{4}(2)$ for $p=3$ by [22]. Note that ${ }^{2} E_{6}(2)$ has abelian Sylow 5- and 7-subgroups, so we are left with $p=3$ and $G / Z(G) \cong{ }^{2} E_{6}(2)$. In this case we do not know the Brauer characters of $G$, so we are forced into a slightly involved argument to make use of the current literature. Note that it suffices to consider the case $|Z(G)|=4$. Our group $G$ has three conjugacy classes of elements of order three, $3 A, 3 B$ and $3 C$. For each such $x \in G$, we have $C_{G}(x) / Z(G) \cong C_{G / Z(G)}(x Z(G))$. Consider a block $B$ covering the block $c$ of $Z(G)$ containing the irreducible character $\lambda$, say. We may assume that $c$ is faithful. By examination of the character table in [14], only two irreducible characters lying over $\lambda$ vanish on $3 A, 3 B$ but not $3 C\left(\chi_{184}\right.$ and $\chi_{202}$ in the notation of [14]). Since a 3 -block of positive defect must possess at least three irreducible characters, it follows by a theorem of Green that $B$ must have a defect group $D$ containing elements of $3 A$ or $3 B$. Suppose $x \in Z(D)$ has order three. Write $Q=\langle x\rangle$. Note that $D C_{G}(D) \leq C_{G}(Q)$, so there is a $B$-subgroup $\left(Q, b_{Q}\right)$ with defect group $D$. If $x \in 3 A$, then $C_{G / Z(G)}(x Z(G)) \cong Q \times P S U_{6}(2)$. We have seen that every block with nonabelian defect groups of a double cover of $P S U_{6}(2)$ has at least two irreducible Brauer characters, so it follows that $l\left(b_{Q}\right)=1$ if $D$ is nonabelian. If $x \in 3 B$, then $C_{G / Z(G)}(x Z(G)) \cong Q \times O_{8}^{+}(2) .3$, and the same argument applies as for $3 A$.

Theorem 9.4 Let $G$ be a quasisimple group and $B$ a nilpotent p-block of $G$ with defect group $D$, where $p$ is odd. Then $D$ is abelian. 
Proof: If $G / Z(G)$ is an alternating group, then the result follows by Theorem 3.3 and the remarks following it. For $G / Z(G)$ sporadic see Theorem 4.5. If $G / Z(G)$ is a classical group and $G$ is a non-exceptional cover, see Propositions 8.1, 8.2, 8.3 and 8.5. For $G / Z(G)$ an exceptional group of Lie type and $G$ is a non-exceptional cover, see Theorem 9.2. For the exceptional covers, see Lemma 9.3.

\section{Puig's conjecture}

We complete the proof of Puig's conjecture for quasisimple groups for odd primes, present some general results and deduce some corollaries.

Theorem 10.1 Let $G$ be a finite quasisimple group and let $B$ be a p-block of $G$ with $p$ odd. Then $B$ is nilpotent if and only if $l\left(b_{Q}\right)=1$ for each $p$-subgroup $Q$ and each block $b_{Q}$ of $C_{G}(Q)$ with $\left(b_{Q}\right)^{G}=B$.

Proof: The necessary condition for nilpotency follows from $[13,1.2]$. By Corollary 7.5 and Propositions 8.1, 8.2, 8.3 and 8.5 the result holds for the classical groups. By Theorem 9.2 it holds for the exceptional groups of Lie type. The result holds for the double covers of the alternating groups by Theorem 3.3, and when $G / Z(G)$ is sporadic by Theorem 4.5. For the exceptional covers of the alternating groups and of the finite simple groups of Lie type, see Lemma 9.3.

Lemma 10.2 Let $N \triangleleft G$ such that $G / N$ is cyclic and of order prime to $p$, and let $B \in \operatorname{Blk}(G)$ cover $b \in \operatorname{Blk}(N)$. Suppose there are abelian $R$ and $P$ and b-subgroups $\left(P, b_{P}\right) \leq\left(R, b_{R}\right)$ such that $b_{P}$ and $b_{R}$ have abelian defect groups and there is $x \in$ $C_{N}(P)$ of order prime to $[G: N]$ such that $x \in N_{N}\left(R, b_{R}\right) \backslash C_{N}(R)$. Then there are $B$-subgroups $\left(P, B_{P}\right) \leq\left(R, B_{R}\right)$ such that $B_{R}$ and $B_{P}$ have abelian defect groups and $x \in N_{G}\left(R, B_{R}\right) \backslash C_{G}(R)$.

Proof: By $[15,4.1]$ there is $B_{R} \in \operatorname{Blk}\left(C_{G}(R)\right)$ such that $\left(B_{R}\right)^{G}=B$ and $B_{R}$ covers $b_{R}$. We claim that the number of such $B_{R}$ divides $[G: N]$. Now $C_{G}(R) / C_{N}(R)$ is cyclic of order dividing $[G: N]$. The blocks of $C_{N}(R)$ and of $C_{G}(R)$ are in 1-1 correspondence with their canonical characters. Let $\theta_{R}$ be the canonical character for $b_{R}$. Since $C_{G}(R) / C_{N}(R)$ is cyclic, $\theta_{R}$ extends to an irreducible character of $C_{G}(R)$, and since $\left[C_{G}(R): C_{N}(R)\right]$ is not divisible by $p$, the extensions are precisely the canonical characters of the blocks of $C_{G}(R)$ covering $b_{R}$. By Clifford theory, the group of irreducible characters of $C_{G}(R) / C_{N}(R)$ acts transitively on the blocks $B_{1}, \ldots, B_{n}$ of $C_{G}(R)$ covering $b_{R}$ by inflation and multiplication, and also transitively on the set $\left\{B_{i}^{G}: 1 \leq i \leq n\right\}$. Consequently the number of blocks of $C_{G}(R)$ covering $b_{R}$ with Brauer correspondent $B$ divides $\left[C_{G}(R): C_{N}(R)\right]$, and the claim follows.

For each $i$, we have $\left(B_{i}^{x}\right)^{G}=\left(B_{i}^{G}\right)^{x}=B^{x}=B$, and $B_{i}^{x}$ overs $b_{i}^{x}=b_{i}$. Hence $x$ permutes $\left\{B_{i}: B_{i}^{G}=B\right\}$. Since this set has order prime to the order of $x$, it follows that $x$ must fix some such $B_{i}$. Call it $B_{R}$. Letting $B_{P}=\left(B_{R}\right)^{C_{G}(P)}$, we are done.

As an almost immediate corollary we have: 
Corollary 10.3 Let $G$ be a finite group such that there is $N \triangleleft G$ with $[G: N]$ odd and $G / N$ is a p-regular cyclic group, where $N$ is quasisimple and $p>3$ is a prime. Let $B$ be a p-block of $G$. Then $B$ is nilpotent if and only if $l\left(b_{Q}\right)=1$ for every $B$-subgroup $\left(Q, b_{Q}\right)$.

Proof: Since the alternating and sporadic groups have outer automorphism groups of order at most two, it follows that it suffices to consider the groups of Lie type. Suppose first that $N$ is not an exceptional covering group. Since $p>3$, every block of $N$ satisfies one of Property 7.1 (a) or (d), and the result follows by Lemma 10.2 and Corollary 7.5.

Suppose that $N$ is an exceptional cover. Then the outer automorphism group is a 2-group except when $N / Z(N) \cong P S L_{3}(4), P S U_{6}(2)$ or ${ }^{2} E_{6}(2)$, in which case it has order three, and consists of diagonal automorphisms. In each case $Z(N)$ is a Klein-four group. However, in each of these cases the non-inner automorphisms transitively permute the blocks whose kernel does not contain $Z(N)$, and the result follows in these cases too, since $B$ is nilpotent if and only if $b$ is, and $B$-subgroups $\left(Q, B_{Q}\right)$ covering $b$-subgroups $\left(Q, b_{Q}\right)$ satisfy $l\left(B_{Q}\right)=l\left(b_{Q}\right)$.

Acknowledgement. The second author thanks the University of Auckland for their hospitality during two visits to the University of Auckland, where part of this work was done.

\section{References}

[1] J. L. Alperin, Large abelian subgroups of p-groups, Tran. Amer. Math. Soc. 117(1965), 10-20.

[2] J. L. Alperin and M. Broué, Local methods in block theory, Ann. Math. 110 (1979), 143-157.

[3] J. An, Weights for classical groups, Tran. Amer. Math. Soc. 342(1994), 1-42.

[4] J. An, J. J. Cannon, E. A. O'Brien and W. R. Unger, The Alperin weight conjecture and Dade's conjecture for the simple group $F i_{24}^{\prime}$, LMS J. Comput. Math. 11(2008), 100-145.

[5] J. An and E. A. O'Brien, The Alperin and Dade conjectures for the Fischer simple group $\mathrm{Fi}_{23}$, Int. J. Algebra and Comput. 9(1999), 621-670.

[6] J. An and E. A. O'Brien, Conjectures on the character degrees of the Harad-Norton simple group $H N$, Israel J. Math. 137(2003), 157-181.

[7] J. An and E. A. O'Brien, The Alperin and Dade conjectures for the Conway simple group $\mathrm{Co}_{1}$, Alg. and Repr. Theory 7(2004), 139-158.

[8] J. An, E. A. O'Brien and R. A. Wilson, The Alperin weight conjecture and Dade's conjecture for the simple group $J_{4}$, LMS J. Comput. Math. 6(2003), 119-140. 
[9] J. An and R. A. Wilson, The Alperin weight conjecture and Uno's conjecture for the baby monster $\mathbb{B}, p$ odd, LMS J. Comput. Math. 7(2004), 120-166.

[10] H. I. Blau and G. O. Michler, Modular representation theory of finite groups with T.I. Sylow p-subgroups, Trans. AMS 319(1990), 417-468.

[11] M. Broué, Les $\ell$-blocs des groupes $\mathrm{GL}(n, q)$ et $\mathrm{U}\left(n, q^{2}\right)$ et leurs structures locales, Séminaire Bourbaki Astérisque 640 (1986), 159-188.

[12] M. Broué and J. Michel, Blocs et séries de Lusztig dans un groupe réductif fini, J. reine angew. Math. 395(1989), 56-67.

[13] M. Broué and L. Puig, A Frobenius theorem for blocks, Invent. Math. 56 (1980), 117-128.

[14] J. H. Conway, R. T. Curtis, S. P. Norton, R. A. Parker and R. A. Wilson, Atlas of Finite Groups (Clarendon Press, Oxford, 1985).

[15] C. W. Eaton, Vertices for irreducible characters of a class of blocks, J. Algebra 286 (2005), 492-499.

[16] P. Fong and B. Srinivasan, The blocks of general linear and unitary groups, Invent. Math 69 (1982), 109-153. 122-191.

[17] P. Fong and B. Srinivasan, The blocks of finite classical groups, J. reine angew. Math. 396 (1989), 122-191.

[18] The GAP Group, GAP - Groups, algorithms, and programming, version 4.4, http://www.gap-system.org, 2005.

[19] F. Garvan, D. Kim and D. Stanton, Cranks and t-cores, Invent. Math. 101(1990), $1-17$.

[20] D. Gorenstein, R. Lyons and R. Solomon, The Classification of Finite Simple Groups. Number 3 (Mathematical Surveys and Monographs, AMS, Providence, 1998).

[21] A. Granville and K. Ono, Defect zero $p$-blocks for finite simple groups, Trans. AMS 348 (1996), 331-347.

[22] G. Hiss, Decomposition matrices of the Chevalley group $F_{4}(2)$ and its covering group, Comm. Algebra 25 (1997), 2539-2555.

[23] G. James and A. Kerber, The representation theory of the symmetric group. (Addison-Wesley, 1981).

[24] B. Külshammer and L. Puig, Extensions of nilpotent blocks, Invent. Math. 102 (1990), 17-71.

[25] H. Nagao and Y. Tsushima, Representations of finite groups, (Academic Press, Inc., Boston, MA, 1989) 
[26] J. B. Olsson, Frobenius symbols for partitions and degrees of spin characters, Math. Scand. 61 (1987), 223-247.

[27] J. B. Olsson, On the $p$-blocks of symmetric and alternating groups and their covering groups, J. Algebra 128 (1990), 188-213.

[28] J. B. Olsson, Combinatorics and representations of finite groups, Vorlesungen aus dem Fachbereich Mathematik der Universität GH Essen 20 (1993).

[29] L. Puig, A survey on the local structure of Morita and Rickard equivalences between Brauer blocks, Representation theory of finite groups (Columbus, OH, 1995), Ohio State Univ. Math. Res. Inst. Publ., 6, de Gruyter (1997), 101-126.

[30] L. Puig and A. Watanabe, On blocks with one simple module in any Brauer correspondenct, J. Algebra 163(1994), 135-138.

[31] M. Sawabe and K. Uno, Conjectures on character degrees for the simple Lyons group, Quart. J. Math. 54 (2003), 103-121.

[32] K. Uno, Conjectures on character degrees for the simple Thompson group, Osaka J. Math. 41 (2004), 11-36.

[33] A. Watanabe, On nilpotent blocks of finite groups, J. Algebra 163(1994), 128-134. 